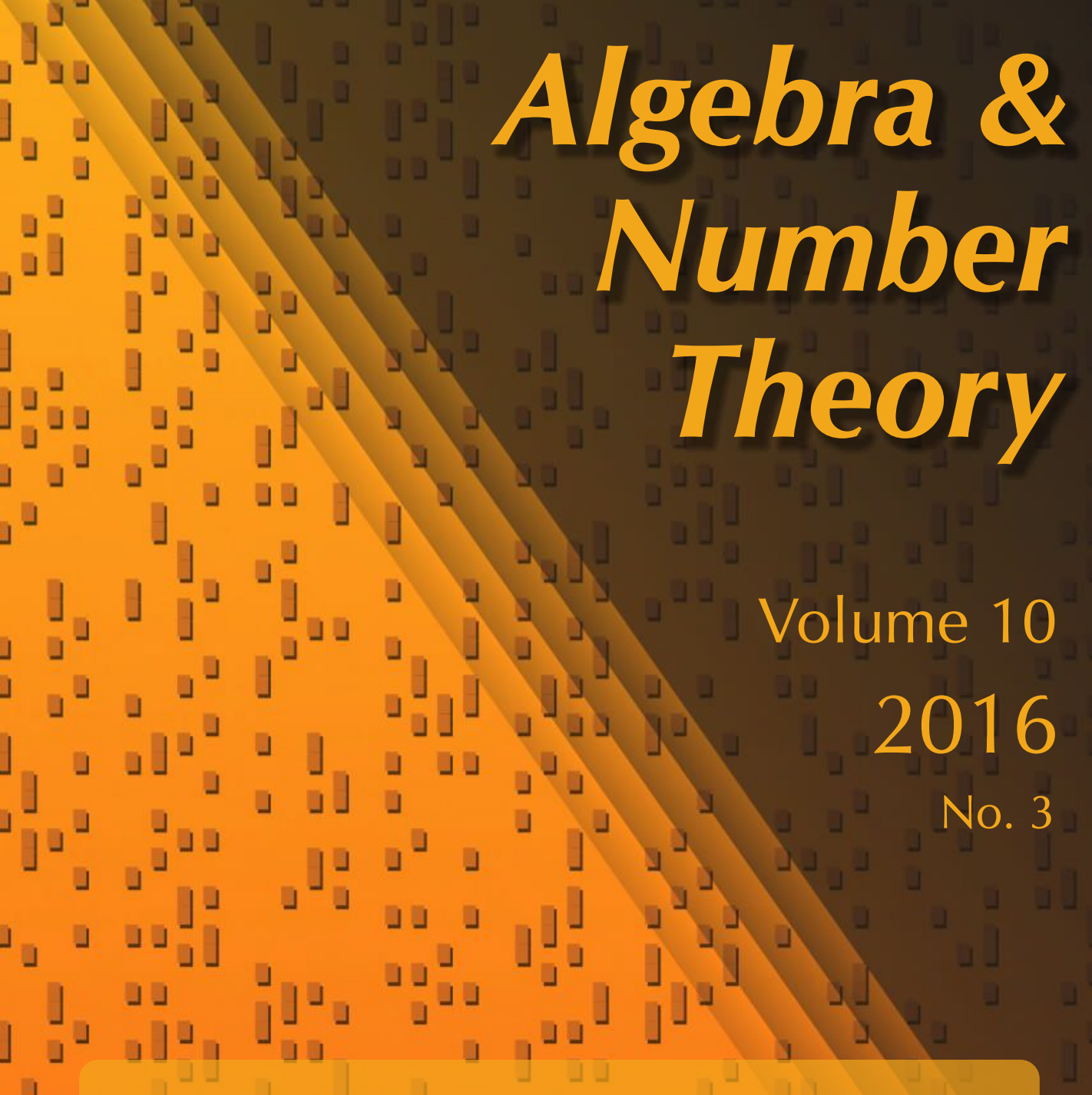

Regularized theta lifts and $(1,1)$-currents on GSpin Shimura varieties

\lrcorner Luis E. Garcia

Jut

ل. 


\title{
Regularized theta lifts and $(1,1)$-currents on GSpin Shimura varieties
}

\author{
Luis E. Garcia
}

We introduce a regularized theta lift for reductive dual pairs $\left(\mathrm{Sp}_{4}, O(V)\right)$, for $V$ a quadratic vector space over a totally real number field. The lift takes values in the space of $(1,1)$-currents on the Shimura variety attached to $\operatorname{GSpin}(V)$; we show its values are cohomologous to currents given by integration on special divisors against automorphic Green functions. A later paper will show how to evaluate the new lift on differential forms obtained as usual (nonregularized) theta lifts.

1. Introduction

2. Shimura varieties and special cycles

3. Currents and regularized theta lifts

606

4. An example: products of Shimura curves

637

Acknowledgments

642

References

\section{Introduction}

1A. Background and main results. The theory of the theta correspondence provides one of the most powerful tools to construct automorphic forms on classical groups. In recent years, the work of many authors has led to a geometric version of this theory describing the behavior of various spaces of so-called special cycles. Namely, the arithmetic quotients of symmetric spaces attached to classical groups $\mathrm{SO}(p, q)$ and $U(p, q)$ are equipped with a large collection of cycles coming from the subgroups that fix a given rational subspace; these are generally known as special cycles. After the work of Kudla and Millson [1986; 1987; 1990] constructing theta functions that represent their Poincaré dual forms, it has become clear that their cohomological properties are very closely connected with the theta correspondence; see, e.g., [Kudla 1997] for a description of their cup products and intersection numbers for the group $\mathrm{SO}(n, 2)$.

In cases where these arithmetic quotients are naturally quasiprojective algebraic varieties (e.g., for the group $\mathrm{SO}(n, 2)$ just mentioned), some of these special cycles

MSC2010: primary 11F27; secondary 11F67, 11G18, 14G35.

Keywords: Shimura varieties, theta series, Weil representation. 
define complex subvarieties, and it is interesting to ask about more refined invariants, such as Green currents for them, or their image in the appropriate Chow groups. The work of Borcherds [1998; 1999] and its generalization by Bruinier [2002; 2012] successfully addressed these questions for the case of special divisors on arithmetic quotients of $\mathrm{SO}(n, 2)$. Their construction relies again on the theta correspondence and is based on considering theta lifts with respect to the reductive dual pair $\left(\mathrm{SL}_{2}, O(V)\right)$. The automorphic forms on $\mathrm{SL}_{2}(\mathbb{A})$ used in their work as an input are not of moderate growth; thus, the integrals defining the theta lifts are not convergent and need to be regularized. With the proper regularization procedure, one can construct Green functions for special divisors, and also meromorphic automorphic forms, as theta lifts.

One might wonder if regularized theta lifts for reductive dual pairs of the form $\left(\mathrm{Sp}_{2 n}, O(V)\right)$ for $n \geq 2$ can be defined and whether one can construct interesting currents on arithmetic quotients of the symmetric space associated with $\operatorname{SO}\left(V_{\mathbb{R}}\right)$ in this way. Consider such a quotient $X_{\Gamma}$ associated with a lattice $\Gamma \subset \operatorname{SO}(n, 2)$, and let $(Y, f)$ be a pair consisting of a subvariety $Y \subset X_{\Gamma}$ and a meromorphic function $f \in \mathbb{C}(Y)^{\times}$. In view of the explicit description of motivic cohomology and regulator maps in terms of higher Chow groups (see, e.g., [Goncharov 2005]), it is interesting to consider the current $\log |f| \cdot \delta_{Y}$, whose value on a differential form $\alpha \in \mathscr{A}_{c}^{*}\left(X_{\Gamma}\right)$ is given by

$$
\left(\log |f| \cdot \delta_{Y}, \alpha\right)=\int_{Y} \log |f| \cdot \alpha .
$$

The first goal of this paper is to show that, for many pairs $(Y, f)$ such that $Y$ is a special subvariety and $f$ has divisor supported in special cycles, the current $\log |f| \cdot \delta_{Y}$ can be obtained as a regularized theta lift for $\left(\mathrm{Sp}_{4}, O(V)\right)$. This follows from Theorem 1.1 below. For motivation, note that conjectures by Beilinson [1984] relate the values of $d d^{c}$-closed $\mathbb{Q}$-linear combinations of such currents with the values at certain integral points of $L$-functions attached to $X_{\Gamma}$. Our construction allows us to compute the values of some more general currents by using the theta correspondence; a followup paper will relate them to special values of standard L-functions of automorphic representations of $\mathrm{Sp}_{4}$. Let us now describe more precisely the main objects involved in the statement of the theorem.

Let $F$ be a totally real number field and $V$ be a quadratic vector space over $F$. We assume that the signature of $V$ is $((n, 2),(n+2,0), \ldots,(n+2,0))$ with $n$ positive and even. Let $H=\operatorname{Res}_{F / \mathbb{Q}} \operatorname{GSpin}(V)$. Attached to $H$ there is a Shimura variety $X$ of dimension $n$ whose complex points at a finite level determined by a neat open compact subgroup $K \subset H\left(\mathbb{A}_{f}\right)$ are given by

$$
X_{K}=H(\mathbb{Q}) \backslash\left(\mathbb{D} \times H\left(\mathbb{A}_{f}\right)\right) / K .
$$


Here $\mathbb{D}$ denotes the hermitian symmetric space attached to the Lie group $\mathrm{SO}\left(V_{\mathbb{R}}\right)$. For fixed $K$, the complex manifold $X_{K}$ is a finite union of arithmetic quotients of the form $X_{\Gamma}:=\Gamma \backslash \mathbb{D}^{+}$, where $\mathbb{D}^{+}$denotes one of the connected components of $\mathbb{D}$. Consider two vectors $v, w \in V$ spanning a totally positive definite plane in $V$ and write $\Gamma_{v}\left(\operatorname{resp} . \Gamma_{v, w}\right)$ for the stabilizer of $v$ (resp. of both $v$ and $w$ ) in $\Gamma$. One can define complex submanifolds $\mathbb{D}_{v}^{+} \subset \mathbb{D}^{+}$and $\mathbb{D}_{v, w}^{+} \subset \mathbb{D}_{v}^{+}$, each of complex codimension one, and holomorphic maps

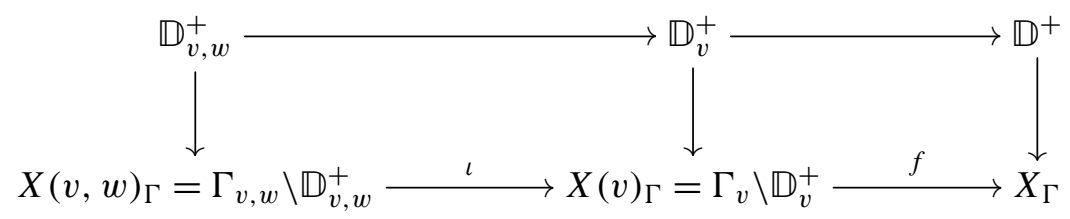

where the maps in the bottom row are proper and generically one-to-one. In Section $3 \mathrm{~B}$ we recall the construction of a function

$$
G(v, w)_{\Gamma} \in \mathscr{C}^{\infty}\left(X(v)_{\Gamma}-\iota\left(X(v, w)_{\Gamma}\right)\right)
$$

that is a Green function for the divisor $\left[\iota\left(X(v, w)_{\Gamma}\right)\right] \in \operatorname{Div}\left(X(v)_{\Gamma}\right)$; this function is locally integrable and hence defines a current $\left[G(v, w)_{\Gamma}\right] \in \mathscr{D}^{0}\left(X(v)_{\Gamma}\right)$. Define the current

$$
\left[\Phi(v, w)_{\Gamma}\right]=2 \pi i \cdot f_{*}\left(\left[G(v, w)_{\Gamma}\right]\right) \in \mathscr{D}^{1,1}\left(X_{\Gamma}\right),
$$

where $f_{*}: \mathscr{D}^{0}\left(X(v)_{\Gamma}\right) \rightarrow \mathscr{D}^{1,1}\left(X_{\Gamma}\right)$ denotes the pushforward map. Note that the $\mathbb{Q}$-linear span of the currents $\left[\Phi(v, w)_{\Gamma}\right]$ for varying $w$ and fixed $v$ includes all the currents of the form $2 \pi i \cdot \log |f| \cdot \delta_{X(v)_{\Gamma}}$, where $f \in \mathbb{C}\left(X(v)_{\Gamma}\right)^{\times} \otimes_{\mathbb{Z}} \mathbb{Q}$ is one of the meromorphic functions constructed by Bruinier [2012, Theorem 6.8]. Given a totally positive definite symmetric matrix $T \in \operatorname{Sym}_{2}(F)$ and a Schwartz function $\varphi \in \mathscr{S}\left(V\left(\mathbb{A}_{f}\right)^{2}\right)$ fixed by $K$, in Section $3 G$ we define a current $\left[\Phi(T, \varphi)_{K}\right] \in$ $\mathscr{D}^{1,1}\left(X_{K}\right)$ as a finite sum of currents $\left[\Phi(v, w)_{\Gamma}\right]$ weighted by the values of $\varphi$. As an example, consider the case treated in Section $4 \mathrm{~B}$, where $X_{K}=X_{0}^{B} \times X_{0}^{B}$ is a self-product of a full level Shimura curve $X_{0}^{B}$ attached to an indefinite quaternion algebra $B$ over $\mathbb{Q}$. Here the currents $\left[\Phi(T, \varphi)_{K}\right]$ admit a description in terms of Hecke correspondences and CM points on $X_{K}$. Namely, if $p$ is a prime not dividing the discriminant of $B$ such that $p \equiv 1(\bmod 4)$, and writing $L=\mathbb{Q}[\sqrt{-p}]$, then for a certain choice of $\varphi=\varphi_{0}$ we have

$$
\left[\Phi\left(\left(\begin{array}{ll}
1 & \\
& p
\end{array}\right), \varphi_{0}\right)_{K}\right]=2 \pi i \cdot\left(X_{0}^{B} \stackrel{\Delta}{\longrightarrow} X_{0}^{B} \times X_{0}^{B}\right)_{*}\left(\left[G_{t_{L / \mathbb{Q}}\left[\operatorname{CM}\left(\mathscr{O}_{L}\right)\right]}\right]\right) .
$$

where $\Delta$ denotes the diagonal embedding and $G_{t_{L / \mathbb{Q}}}\left[\mathrm{CM}\left(\mathscr{O}_{L}\right)\right]$ denotes a Green function for the divisor $t_{L / \mathbb{Q}}\left[\mathrm{CM}\left(\mathscr{O}_{L}\right)\right]$ of points in $X_{0}^{B}$ with $\mathrm{CM}$ by $\mathscr{O}_{L}$ (see (4-28)). 
Our first main result will show that the currents $\left[\Phi(T, \varphi)_{K}\right]$ are cohomologous to some currents obtained by a process of regularized theta lifting. Let us now introduce these theta lifts. In Section $3 \mathrm{H}$ we define, for $\varphi \in \mathscr{S}\left(V\left(\mathbb{A}_{f}\right)^{2}\right)$ fixed by $K$ and $g \in \mathrm{Sp}_{4}\left(\mathbb{A}_{F}\right)$, a theta function $\theta(g ; \varphi)_{K}$ valued in the space of smooth $(1,1)$-forms on $X_{K}$. In the same section, we introduce a function

$$
\mathscr{M}_{T}(s): N(F) \backslash N(\mathbb{A}) \times A(\mathbb{R})^{0} \longrightarrow \mathbb{C} .
$$

Here $T$ denotes a totally positive definite symmetric 2-by-2 matrix, $s$ is a complex number, $N \subset \mathrm{Sp}_{4, F}$ denotes the unipotent radical of the Siegel parabolic of $\mathrm{Sp}_{4, F}$ and $A(\mathbb{R})^{0}$ denotes the connected component of the identity of the real points of the subgroup $A \subset \mathrm{Sp}_{4, F}$ of diagonal matrices in $\mathrm{Sp}_{4, F}$. This function grows exponentially along $A(\mathbb{R})^{0}$. We define the regularized theta lift

$$
\left(\mathscr{M}_{T}(s), \theta(\cdot, \varphi)_{K}\right)^{\mathrm{reg}}=\int_{A(\mathbb{R})^{0}} \int_{N(F) \backslash N(\mathbb{A})} \mathscr{M}_{T}(n a, s) \theta(n a, \varphi)_{K} d n d a,
$$

with appropriate measures $d n$ and $d a$.

Theorem 1.1. (1) The regularized integral $\left(\mathscr{M}_{T}(s), \theta(\cdot ; \varphi)_{K}\right)^{\text {reg }}$ converges for $\operatorname{Re}(s) \gg 0$ on an open dense set of $X_{K}$ whose complement has measure zero and defines a locally integrable $(1,1)$-form $\Phi(T, \varphi, s)_{K}$ on $X_{K}$.

(2) Let $\tilde{\mathscr{D}}^{1,1}\left(X_{K}\right)=\mathscr{D}^{1,1}\left(X_{K}\right) /(\operatorname{Im}(\partial)+\operatorname{Im}(\bar{\partial}))$. The current $\left[\Phi(T, \varphi, s)_{K}\right] \in$ $\tilde{\mathscr{D}}^{1,1}\left(X_{K}\right)$ defined by $\Phi(T, \varphi, s)_{K}$ admits meromorphic continuation to $s \in \mathbb{C}$; moreover, its constant term at $s=s_{0}=(n-1) / 2$ satisfies

$$
\mathrm{CT}_{s=s_{0}}\left[\Phi(T, \varphi, s)_{K}\right]=\left[\Phi(T, \varphi)_{K}\right]
$$

as elements of $\tilde{D}^{1,1}\left(X_{K}\right)$.

In fact, Proposition 3.19 shows that the currents in the theorem are compatible under the maps $\mathscr{D}^{1,1}\left(X_{K^{\prime}}\right) \rightarrow \mathscr{D}^{1,1}\left(X_{K}\right)$ induced from inclusions $K^{\prime} \subset K$ of open compact subgroups, so that we obtain currents

$$
[\Phi(T, \varphi)]=\left(\left[\Phi(T, \varphi)_{K}\right]\right)_{K} \in \mathscr{D}^{1,1}(X):={\underset{K}{K}}_{\lim ^{1}} \mathscr{D}^{1,1}\left(X_{K}\right)
$$

and similarly $[\Phi(T, \varphi, s)] \in \mathscr{D}^{1,1}(X)$ that agree on closed differential forms.

A particularly interesting subspace of $\mathscr{D}^{1,1}\left(X_{K}\right)$ is the image of the regulator map

$$
r_{\mathscr{D}}: \mathrm{CH}^{2}\left(X_{K}, 1\right) \rightarrow \mathscr{D}^{1,1}\left(X_{K}\right)
$$

whose definition we recall in Section 3I; in particular, we would like to characterize the currents $\left[\Phi_{K}\right]$ in the $\mathbb{Q}$-linear span of the currents $\left[\Phi(T, \varphi)_{K}\right]$ that belong to the 
image of $r_{\mathscr{D}}$. We will prove in Proposition 3.23 that, when $\operatorname{dim} X_{K} \geq 4$, we have for such a current $\Phi_{K}$,

$$
\left[\Phi_{K}\right] \in r_{\mathscr{D}} \Longleftrightarrow d d^{c}\left[\Phi_{K}\right]=0 .
$$

Let us assume from now on that $V$ is anisotropic over $F$; this implies that $X_{K}$ is compact. Once the currents $[\Phi(T, \varphi)]$ have been constructed, we would like to evaluate them on differential forms $\alpha \in \mathscr{A}_{c}^{n-1, n-1}\left(X_{K}\right)$. Since the form $\Phi(T, \varphi, s)_{K}$ is obtained as a (regularized) integral, it is natural to try to do so by interchanging the integrals. However, the regularized integral is not absolutely convergent, and the exchange is not justified. To get around this problem, we introduce some locally integrable (1,1)-forms $\tilde{\Phi}(T, \varphi, s)_{K}$ related to the $\Phi(T, \varphi, s)_{K}$ in Theorem 1.1. They are also obtained as regularized theta lifts and the associated currents $\left[\tilde{\Phi}(T, \varphi, s)_{K}\right]$ are compatible under the maps induced by inclusions $K^{\prime} \subset K$, thus defining a current $[\tilde{\Phi}(T, \varphi, s)] \in \mathscr{D}^{1,1}(X)$. As before, these currents enjoy a property of meromorphic continuation to $s \in \mathbb{C}$, and their constant terms satisfy

$$
\mathrm{CT}_{s=s_{0}}\left[\tilde{\Phi}\left(T_{1}, \varphi_{1}, s\right)\right]-\left[\tilde{\Phi}\left(T_{2}, \varphi_{2}, s\right)\right] \equiv\left[\Phi\left(T_{1}, \varphi_{1}\right)\right]-\left[\Phi\left(T_{2}, \varphi_{2}\right)\right]
$$

modulo $\operatorname{Im}(\partial)+\operatorname{Im}(\bar{\partial})$ for pairs $\left(T_{1}, \varphi_{1}\right),\left(T_{2}, \varphi_{2}\right)$ related by a certain involution $\iota$ (see (3-82)). Here, at a finite level $K$, the current on the right hand side is a finite sum of currents of the form $\left[\Phi(v, w)_{\Gamma}\right]-\left[\Phi(w, v)_{\Gamma}\right]$, with $\left[\Phi(v, w)_{\Gamma}\right]$ given by (1-4); see Remark 3.24 for some motivation on these currents. Moreover, using ideas of Bruinier and Funke [2004], we show that the values $\left[\tilde{\Phi}(T, \varphi, s)_{K}\right](\alpha)$ for large $\operatorname{Re}(s)$ can be computed by reversing the order of integration; the precise statement is the following.

Proposition 3.27. Let $K \subset H\left(\mathbb{A}_{f}\right)$ be an open compact subgroup that fixes $\varphi$ and let $\alpha \in \mathscr{A}_{c}^{n-1, n-1}\left(X_{K}\right)$. Then, for $\operatorname{Re}(s) \gg 0$, we have

$\left(\left[\tilde{\Phi}(T, \varphi, s)_{K}\right], \alpha\right)=\int_{A(\mathbb{R})^{0}} \int_{N(F) \backslash N(\mathbb{A})} \tilde{\mathscr{M}}_{T}(n a, s) \int_{X_{K}} \theta\left(n a ; \varphi \otimes \tilde{\varphi}_{\infty}\right)_{K} \wedge \alpha d n d a$.

This result also gives information on the values of the currents $[\Phi(T, \varphi)]$; see Corollary 3.28.

1B. Outline of the paper. We now describe the contents of each section in more detail. Section 2 is a review of definitions and basic facts about Shimura varieties $X$ attached to GSpin groups. In it we recall the definition of the relevant Shimura datum, describe the connected components of $X_{K}$ at a finite level $K$ and introduce the tautological line bundle $\mathscr{L}$ and its canonical metric. Then we recall the definition of special cycles in $X_{K}$ and their weighted versions introduced by Kudla.

In Section 3 we construct currents in $\mathscr{D}^{1,1}\left(X_{K}\right)$. Sections $3 \mathrm{~A}$ and $3 \mathrm{~B}$ first review previous work by Oda, Tsuzuki and Bruinier on secondary spherical functions on 
the symmetric space $\mathbb{D}$ attached to $\mathrm{SO}(n, 2)$, and on automorphic Green functions for special divisors on arithmetic quotients $\Gamma \backslash \mathbb{D}^{+}$(here $\mathbb{D}^{+}$denotes one of the connected components of $\mathbb{D}$ ). In Section $3 \mathrm{C}$ we introduce some differential forms with singularities on $\mathbb{D}$. These forms depend on a complex parameter $s$ and are used in Section 3D to define $(1,1)$-forms on $\Gamma \backslash \mathbb{D}^{+}$with singularities on special divisors. We prove that these $(1,1)$-forms are locally integrable and therefore define currents in $\mathscr{D}^{1,1}\left(\Gamma \backslash \mathbb{D}^{+}\right)$. Section $3 \mathrm{E}$ then shows that these currents admit meromorphic continuation to $s \in \mathbb{C}$ and that their regularized value at a certain value $s_{0}$ is cohomologous to the pushforward of the automorphic Green function in Section 3B defined on a certain special divisor. An adelic formulation of the above constructions is provided in Section 3F. After this, in Section 3G, we introduce weighted currents; their behavior under pullbacks induced by inclusions of open compact subgroups $K^{\prime} \subset K$ and under the Hecke algebra of the GSpin group is described. Section $3 \mathrm{H}$ explains how these weighted currents can be constructed as regularized theta lifts for the dual pair $\left(\mathrm{Sp}_{4}, O(V)\right)$. In Section 3I we give a necessary and sufficient condition for the currents above to belong to the image of the regulator map from the higher Chow group $\mathrm{CH}^{2}\left(X_{K}, 1\right)$. Section 3J introduces some related currents on $X_{K}$ and uses their presentation as regularized theta lifts to prove that they can be evaluated on differential forms by interchanging the order of integration.

The example of a product of Shimura curves described above is considered in Section 4. This section starts with some definitions and basic facts on Shimura curves in Section 4A. In Section 4B, we describe several of the currents introduced in Section 3 in terms of Hecke correspondences and CM divisors.

1C. Notation. The following conventions will be used throughout the paper.

- We write $\hat{\mathbb{Z}}=\lim _{n}(\mathbb{Z} / n \mathbb{Z})$ and $\hat{M}=M \otimes_{\mathbb{Z}} \hat{\mathbb{Z}}$ for any abelian group $M$. We write $\mathbb{A}_{f}=\mathbb{Q} \otimes_{\mathbb{Z}} \hat{\mathbb{Z}}$ for the finite adeles of $\mathbb{Q}$ and $\mathbb{A}=\mathbb{A}_{f} \times \mathbb{R}$ for the full ring of adeles.

- For a number field $F$, we write $\mathbb{A}_{F}=F \otimes_{\mathbb{Q}} \mathbb{A}, \mathbb{A}_{F, f}=F \otimes_{\mathbb{Q}} \mathbb{A}_{f}$ and $F_{\infty}=$ $F \otimes_{\mathbb{Q}} \mathbb{R}$. We will suppress $F$ from the notation if no ambiguity can arise.

- For a finite set of places $S$ of $F$, we will denote by $\mathbb{A}_{S}$ and $\mathbb{A}^{S}$ the subset of adeles in $\mathbb{A}_{F}$ supported on $S$ and away from $S$, respectively.

- We denote by $\psi_{\mathbb{Q}}=\bigotimes_{v} \psi_{\mathbb{Q}_{v}}: \mathbb{Q} \backslash \mathbb{A}_{\mathbb{Q}} \rightarrow \mathbb{C}^{\times}$the standard additive character of $\mathbb{A}_{\mathbb{Q}}$, defined by

$$
\begin{aligned}
\psi_{\mathbb{Q}_{p}}(x)=e^{-2 \pi i x} & \text { for } x \in \mathbb{Z}\left[p^{-1}\right], \\
\psi_{\mathbb{R}}(x)=e^{2 \pi i x} & \text { for } x \in \mathbb{R} .
\end{aligned}
$$


If $F_{v}$ is a finite extension of $\mathbb{Q}_{v}$, we set $\psi_{v}=\psi_{\mathbb{Q}_{v}}(\operatorname{tr}(x))$, where $\operatorname{tr}: F_{v} \rightarrow \mathbb{Q}_{v}$ is the trace map. For a number field $F$, we write $\psi=\bigotimes_{v} \psi_{v}: F \backslash \mathbb{A}_{F} \rightarrow \mathbb{C}^{\times}$ for the resulting additive character of $\mathbb{A}_{F}$.

- For a locally compact, totally disconnected topological space $X$, the symbol $\mathscr{S}(X)$ denotes the Schwartz space of locally constant, compactly supported functions on $X$. For $X$ a finite dimensional vector space over $\mathbb{R}$, the symbol $\mathscr{S}(X)$ denotes the Schwartz space of all $\mathscr{C}^{\infty}$ functions on $X$ all whose derivatives are rapidly decreasing.

- For a ring $R$, we denote by $\operatorname{Mat}_{n}(R)$ the set of all $n$-by- $n$ matrices with entries in $R$. The symbols $1_{n}$ and $0_{n}$ denote the identity and zero matrices in $\operatorname{Mat}_{n}(R)$.

- The transpose of a matrix $x \in \operatorname{Mat}_{n}(R)$, is denoted ${ }^{t} x$, and the set of all symmetric matrices in $\operatorname{Mat}_{n}(R)$ is $\operatorname{Sym}_{n}(R)=\left\{x \in \operatorname{Mat}_{n}(R) \mid x={ }^{t} x\right\}$.

- $X \amalg Y$ denotes the disjoint union of $X$ and $Y$.

- If an object $\phi(s)$ depends on a complex parameter $s$ and is meromorphic in $s$, we denote by $\mathrm{CT}_{s=s_{0}} \phi(s)$ the constant term of its Laurent expansion at $s=s_{0}$.

\section{Shimura varieties and special cycles}

2A. Shimura varieties. We recall the facts about orthogonal Shimura varieties that we will need. We follow [Kudla 1997] closely, to which the reader is referred for further details. Let $F$ be a totally real number field of degree $d$ with embeddings $\sigma_{i}: F \rightarrow \mathbb{R}, i=1, \ldots, d$. Let $(V, Q)$ a quadratic vector space over $F$ of dimension $n+2$ (with $n \geq 1$ ); we assume that $V_{1}=V \otimes_{F, \sigma_{1}} \mathbb{R}$ has signature $(n, 2)$ and that $V_{\sigma_{i}}=V \otimes_{F, \sigma_{i}} \mathbb{R}$ is positive definite for $i=2, \ldots, d$.

Let $H=\operatorname{Res}_{F / \mathbb{Q}} \operatorname{GSpin}(V)$. The group $H$ fits into a short exact sequence

$$
1 \longrightarrow \operatorname{Res}_{F / \mathbb{Q}} \mathbb{G}_{m} \longrightarrow H \longrightarrow \operatorname{Res}_{F / \mathbb{Q}} \operatorname{SO}(V) \longrightarrow 1 \text {. }
$$

Denote by $\mathbb{D}$ the set of oriented negative definite planes in $V_{1}$. We will fix once and for all a point $z_{0} \in \mathbb{D}$ and will denote by $\mathbb{D}^{+}$the connected component of $\mathbb{D}$ containing $z_{0}$. The group $\mathrm{SO}\left(V_{1}\right) \cong \mathrm{SO}(n, 2)$ acts transitively on $\mathbb{D}$, and the stabilizer $K_{z_{0}}$ of $z_{0}$ is isomorphic to $\mathrm{SO}(n) \times \mathrm{SO}(2)$. We have

$$
\mathbb{D} \cong \mathrm{SO}(n, 2) /(\mathrm{SO}(n) \times \mathrm{SO}(2)) .
$$

To the pair $(H, \mathbb{D})$ one can attach a Shimura variety $\operatorname{Sh}(H, \mathbb{D})$ that has a canonical model over $\sigma_{1}(F)$. Namely, in [Kudla 1997, p. 44] a homomorphism

$$
h_{0}: \operatorname{Res}_{\mathbb{C} / \mathbb{R}} \mathbb{G}_{m}=\mathbb{C}^{\times} \longrightarrow H(\mathbb{R})=\prod_{i=1, \ldots, d} \operatorname{GSpin}\left(V_{\sigma_{i}}\right)
$$


is defined such that $\mathbb{D}$ becomes identified with the space of conjugates of $h_{0}$ by $H(\mathbb{R})$; the resulting action of $H(\mathbb{R})$ on $\mathbb{D}$ factors through the projection $H(\mathbb{R}) \rightarrow \mathrm{SO}\left(V_{1}\right)$. For any compact open subgroup $K \subset H\left(\mathbb{A}_{f}\right)$, we have

$$
X_{K}=\operatorname{Sh}(H, \mathbb{D})_{K}(\mathbb{C})=H(\mathbb{Q}) \backslash\left(\mathbb{D} \times H\left(\mathbb{A}_{f}\right)\right) / K .
$$

Thus $X_{K}$ is the complex analytification of a quasiprojective variety $\operatorname{Sh}(H, \mathbb{D})_{K}$ of dimension $n$ defined over $\sigma_{1}(F)$. If $V$ is anisotropic over $F$, then $\operatorname{Sh}(G, \mathbb{D})_{K}$ is actually projective.

We recall the description of the connected components of $X_{K}$. Let $H^{\text {der }} \cong$ $\operatorname{Res}_{F / \mathbb{Q}} \operatorname{Spin}(V)$ be the derived subgroup of $H$. There is an exact sequence

$$
1 \longrightarrow H^{\mathrm{der}} \longrightarrow H \stackrel{v}{\longrightarrow} T \longrightarrow 1,
$$

where $T=\operatorname{Res}_{F / \mathbb{Q}} \mathbb{G}_{m}$ and $v$ is given by the spinor norm. Let $T(\mathbb{R})^{+}=\left(\mathbb{R}_{>0}\right)^{d} \subset$ $T(\mathbb{R})$ and $H_{+}(\mathbb{R})=v^{-1}\left(T(\mathbb{R})^{+}\right)$be the set of elements of $H(\mathbb{R})$ of totally positive spinor norm; this is the subgroup of $H(\mathbb{R})$ stabilizing $\mathbb{D}^{+}$. Define

$$
H_{+}(\mathbb{Q})=H(\mathbb{Q}) \cap H_{+}(\mathbb{R}) .
$$

By the strong approximation theorem, we can find $h_{1}=1, \ldots, h_{r} \in H\left(\mathbb{A}_{f}\right)$ such that

$$
H\left(\mathbb{A}_{f}\right)=\coprod_{j=1}^{r} H_{+}(\mathbb{Q}) h_{j} K .
$$

For $j=1, \ldots, r$, let $\Gamma_{h_{j}}=H_{+}(\mathbb{Q}) \cap h_{j} K h_{j}^{-1}$. Then

$$
X_{K} \cong \coprod_{j=1}^{r} \Gamma_{h_{j}} \backslash \mathbb{D}^{+} .
$$

We will also need to consider Shimura varieties attached to $(V, Q)$ as above with $n=0$. In this case, the symmetric domain associated with $\operatorname{SO}\left(V_{1}\right)$ consists of just one point, while $\mathbb{D}=\mathbb{D}^{+} U \mathbb{D}^{-}$consists of two points (corresponding to two different orientations of the same negative definite plane $z_{0}$ ). Since it turns out to be more convenient for our purposes, we define $X_{K}$ as in (2-4) and $\operatorname{Sh}(H, \mathbb{D})_{K}$ to be the union of two copies of the usual Shimura variety attached to $H$, so that with these notations we have $X_{K}=\operatorname{Sh}(H, \mathbb{D})_{K}(\mathbb{C})$.

For $n \geq 1$, we can introduce a different model for $\mathbb{D}$ that makes the presence of an $\mathrm{SO}\left(V_{1}\right)$-invariant complex structure obvious. Let $\mathscr{Q}$ be the quadric in $\mathbb{P}\left(V_{1}(\mathbb{C})\right)$ given by

$$
\mathscr{Q}=\left\{v \in \mathbb{P}\left(V_{1}(\mathbb{C})\right) \mid(v, v)=0\right\} .
$$

Note that if $\left\{v_{1}, v_{2}\right\}$ is an orthogonal basis of $z \in \mathbb{D}$ with $\left(v_{1}, v_{1}\right)=\left(v_{2}, v_{2}\right)=-1$, then $v:=v_{1}-i v_{2} \in V_{1} \otimes \mathbb{C}$ satisfies $(v, v)=0$ and $(v, \bar{v})<0$. Moreover, the line 
$[v]:=\mathbb{C} \cdot v$ is independent of the orthogonal basis we have chosen. Thus we obtain a well defined map $\mathbb{D} \rightarrow \mathscr{Q}$ and one checks that it gives an isomorphism

$$
\mathbb{D} \longrightarrow \mathscr{Q}_{-}=\left\{w \in \mathbb{P}\left(V_{\sigma_{1}}(\mathbb{C})\right) \mid(w, w)=0,(w, \bar{w})<0\right\}
$$

onto the open subset $\mathscr{Q}_{-}$of the quadric $\mathscr{Q}$.

Consider the tautological line bundle $\mathscr{L}$ over $\mathscr{Q}_{-}$defined by

$$
\mathscr{L} \backslash\{0\}:=\left\{w \in V_{1}(\mathbb{C}) \mid(w, w)=0,(w, \bar{w})<0\right\} .
$$

The action of $H(\mathbb{R})$ on $\mathbb{D}$ lifts naturally to $\mathscr{L}$ and gives it the structure of a $H(\mathbb{R})$ equivariant bundle. Any element $v \in V_{1}$ defines a section $s_{v}$ of $\mathscr{L}^{\vee}$ by the rule $s_{v}(w)=(v, w)$. We will only consider $s_{v}$ for $v$ of positive norm. The section $s_{v}$ defines an analytic divisor

$$
\operatorname{div}\left(s_{v}\right)=\left\{w \in \mathbb{P}\left(V_{1}(\mathbb{C})\right) \mid(v, w)=0\right\} .
$$

Under the isomorphism $\mathbb{D} \cong \mathscr{Q}_{-}$described above, div $s_{v}$ corresponds to $\mathbb{D}_{v} \subset \mathbb{D}$, where $\mathbb{D}_{v}$ denotes the set of negative definite planes in $V_{1}$ that are orthogonal to $v$.

The line bundle $\mathscr{L}$ carries a natural hermitian metric $\|\cdot\|$ defined by $\|w\|^{2}=$ $|(w, \bar{w})|$; this metric is $H(\mathbb{R})$-equivariant. We say that a function $f \in \mathscr{C}^{\infty}\left(\mathbb{D}-\mathbb{D}_{v}\right)$ has a logarithmic singularity along $\mathbb{D}_{v}$ if $f(z)-\log \left\|s_{v}(z)\right\|^{2}$ extends to $\mathscr{C}^{\infty}(\mathbb{D})$.

2B. Special cycles. Let $U \subset V$ be a totally positive definite subspace and let $W$ be its orthogonal complement in $V$. Denote by $H_{U}$ the pointwise stabilizer of $U$ in $H$. Then $H_{U} \cong \operatorname{Res}_{F / \mathbb{Q}} \operatorname{GSpin}(W)$; its associated symmetric domain can be identified with $\mathbb{D}_{U} \cap \mathbb{D}^{+}$, where $\mathbb{D}_{U}$ denotes the subset of $\mathbb{D}$ consisting of planes $z$ that are orthogonal to $U$. For a compact open $K \subset H\left(\mathbb{A}_{f}\right)$ and $h \in H\left(\mathbb{A}_{f}\right)$, let $K_{U, h}=H_{U}\left(\mathbb{A}_{f}\right) \cap h K h^{-1}$, an open compact subset of $H_{U}\left(\mathbb{A}_{f}\right)$. Define

$$
X(U, h)_{K}=H_{U}(\mathbb{Q}) \backslash\left(\mathbb{D}_{U} \times H_{U}\left(\mathbb{A}_{f}\right)\right) / K_{U, h} .
$$

If $h=1$, we write $X(U)_{K}:=X(U, 1)_{K}$. Thus $X(U, h)_{K}$ is the set of complex points of a variety $\operatorname{Sh}\left(H_{U}, \mathbb{D}_{U}\right)_{K_{U, h}}$ defined over $\sigma_{1}(F)$. There is a morphism

$$
i_{U}: \operatorname{Sh}\left(H_{U}, \mathbb{D}_{U}\right) \longrightarrow \operatorname{Sh}(H, \mathbb{D})
$$

defined over $\sigma_{1}(F)$; on complex points it induces a map

$$
i_{U, h, K}: X(U, h)_{K} \longrightarrow X_{K}
$$

that is proper and birational onto its image. Denote by $Z(U, h)_{K}$ the associated effective cycle on $X_{K}$. For a set of vectors $x=\left(x_{1}, \ldots, x_{r}\right) \in V^{r}$ spanning a totally positive definite vector space $U$ of dimension $r$, we will write $Z(x, h)_{K}$ for $Z(U, h)_{K}$. 
For a description of the connected components of these special cycles, see [Kudla 1997, Sections 3 and 4]; the main result is that these cycles have a finite number of components of the form $Z(U, h)_{\Gamma}$ that we now define. For $h \in H\left(\mathbb{A}_{f}\right)$, let $\Gamma_{h}=H_{+}(\mathbb{Q}) \cap h K h^{-1}$. Define $\Gamma_{U, h}=\Gamma_{h} \cap H_{U}(\mathbb{R})$ and consider the map

$$
X(U, h)_{\Gamma}:=\Gamma_{U, h} \backslash \mathbb{D}_{U}^{+} \longrightarrow \Gamma_{h} \backslash \mathbb{D}^{+}=X_{\Gamma_{h}} .
$$

(For $h=1$, we will just write $X(U)_{\Gamma}$ for $\left.X(U, 1)_{\Gamma}\right)$. The image defines a connected cycle in $X_{\Gamma_{h}}$ that we denote by $Z(U, h)_{\Gamma}$.

In [Kudla 1997], certain weighted sums of these cycles are defined. Namely, let $r=\operatorname{dim}_{F} U$ and denote by $\operatorname{Sym}_{r}(F)_{>0}$ the space of totally positive definite $r$-by- $r$ matrices with coefficients in $F$. For $T \in \operatorname{Sym}_{r}(F)_{>0}$ and $\varphi \in \mathscr{S}\left(V\left(\mathbb{A}_{f}\right)^{r}\right)^{K}$ with values in a ring $R$, define

$$
Z(T, \varphi)_{K}=\sum_{h \in H_{U}\left(\mathbb{A}_{f}\right) \backslash H\left(\mathbb{A}_{f}\right) / K} \varphi\left(h^{-1} x\right) Z(x, h)_{K},
$$

where $x=\left(x_{1}, \ldots, x_{r}\right) \in V^{r}$ is any vector with $\frac{1}{2}\left(x_{i}, x_{j}\right)=T$ (if no such $x$ exists, we set $Z(T, \varphi)=0)$. Note that the sum is finite and hence defines a cycle in $Z^{r}\left(X_{K}\right) \otimes_{\mathbb{Z}} R$.

\section{Currents and regularized theta lifts}

In this section we introduce some differential forms and currents on arithmetic quotients of $\mathbb{D}^{+}$. Some of these forms will be defined as Poincaré series by summation of $\Gamma$-translates of a differential form on $\mathbb{D}^{+}$. Here and throughout this paper, $\Gamma \subset H_{+}(\mathbb{R})$ denotes a group of the form $\Gamma=H_{+}(\mathbb{Q}) \cap K$, where $K \subset H\left(\mathbb{A}_{f}\right)$ is some neat open compact subgroup. If $U \subset V$ is a totally positive definite subspace, we will write $\Gamma_{U}=\Gamma \cap H_{U}(\mathbb{R})$, where $H_{U}$ denotes the pointwise stabilizer of $U$ in $H$. If $U$ is spanned by vectors $v_{1}, \ldots, v_{r}$, we will sometimes write $\Gamma_{v_{1}, \ldots, v_{r}}$ for $\Gamma_{U}$.

Several currents defined in this Section will be described explicitly in Section 4B, where we consider the particular case when $X_{K}$ is a product of Shimura curves. The description given there is in terms of Hecke correspondences and CM points, and the reader is advised to study the examples given there to understand the definitions and properties to follow.

3A. Secondary spherical functions on $\mathbb{D}$. Recall that $\mathbb{D}$ denotes the set of oriented, negative definite 2-planes in $V_{1}=V \otimes_{F, \sigma_{1}} \mathbb{R}$. For every vector $v \in V_{1}$ of positive norm we have defined an analytic divisor $\mathbb{D}_{v} \subset \mathbb{D}$ consisting of those $z \in \mathbb{D}$ that are orthogonal to $v$. Denote by $H_{v}(\mathbb{R})$ the stabilizer of $v$ in $H(\mathbb{R})$. Then we have $\mathbb{D}_{v} \cong H_{v}(\mathbb{R}) /\left(K \cap H_{v}(\mathbb{R})\right)$, so that $\mathbb{D}_{v}$ can be identified with the hermitian symmetric space associated with $H_{v}(\mathbb{R})$. We write $\mathbb{D}_{v}^{+}:=\mathbb{D}_{v} \cap \mathbb{D}^{+}$. 
We recall some of the main results of Oda and Tsuzuki [2003] concerning the existence and main properties of secondary spherical functions on $\mathbb{D}$. To state these results, we need to introduce certain subgroups of $G=\operatorname{SO}\left(V_{1}\right)$. Let $\left\{v_{1}, \ldots, v_{n+2}\right\}$ be a basis of $V_{1}$ whose quadratic form is $I_{n, 2}$ and such that $v=v_{1}$. Let $z_{0}=\left\langle v_{n+1}, v_{n+2}\right\rangle$ and denote by $K_{z_{0}}$ the stabilizer of $z_{0}$ in $\mathrm{SO}\left(V_{1}\right)^{+}$. Let $W \subset V_{1}$ be the plane generated by $v_{1}$ and $v_{n+1}$ and let $A=\mathrm{SO}(W)^{0}$ be the identity component of its orthogonal group. Then $A=\left\{a_{t} \mid t \in \mathbb{R}\right\}$ where $a_{t} v_{1}=\cosh (t) v_{1}+\sinh (t) v_{n+1}$. Let

$$
A^{+}=\left\{a_{t} \mid t \geq 0\right\}
$$

and $G_{v}$ be the stabilizer of $v$ in $G$. Then there is a double coset decomposition

$$
G=G_{v} A^{+} K_{z_{0}}
$$

Proposition 3.1 [Oda and Tsuzuki 2003, Proposition 2.4.2]. Let $\Delta_{\mathbb{D}}$ be the invariant Laplacian on $\mathbb{D}$ and let $\rho_{0}=n / 2$. Let $s$ be a complex number with $\operatorname{Re}(s)>\rho_{0}$. There exists a unique function $\phi^{(2)}(v, z, s) \in \mathscr{C}^{\infty}\left(\mathbb{D}-\mathbb{D}_{v}\right)$ with the following properties:

(1) $\Delta_{\mathbb{D}} \phi^{(2)}(v, z, s)=\left(s^{2}-\rho_{0}^{2}\right) \phi^{(2)}(v, z, s)$.

(2) $\phi^{(2)}(v, g z, s)=\phi^{(2)}(v, z, s)$ for every $g \in G_{v}$.

(3) Consider the function $\phi^{(2)}(v, g, s)=\phi^{(2)}\left(v, g z_{0}, s\right)$ for $g \in G$. It belongs to $\mathscr{C}^{\infty}\left(G-G_{v} K_{z_{0}}\right)$ and satisfies $\phi^{(2)}\left(v, g^{\prime} g k, s\right)=\phi^{(2)}(v, g, s)$ for every $g^{\prime} \in G_{v}$, $k \in K_{z_{0}}$. Writing $G=G_{v} A^{+} K_{z_{0}}$ as above, we have

$$
\begin{array}{ll}
\phi^{(2)}\left(v, a_{t}, s\right)=\log (t)+O(1) & \text { as } t \rightarrow 0, \\
\phi^{(2)}\left(v, a_{t}, s\right)=O\left(e^{-\left(\operatorname{Re}(s)+\rho_{0}\right) t}\right) & \text { as } t \rightarrow+\infty .
\end{array}
$$

It follows that $\phi^{(2)}(h v, h z, s)=\phi^{(2)}(v, z, s)$ for all $h \in H(\mathbb{R})$ and $z \in \mathbb{D}$. For a totally positive vector $v \in V(F)$, we will simply write $\phi^{(2)}(v, z, s)$ for $\phi^{(2)}\left(v_{1}, z, s\right)$, where $v_{1}$ denotes the image of $v$ in $V_{1}$. We will sometimes write $\phi_{\mathbb{D}}^{(2)}(v, z, s)$ for $\phi^{(2)}(v, z, s)$ if we need to be precise about the domain of definition.

The function $\phi^{(2)}(v, z, s)$ admits an explicit description in terms of the Gaussian hypergeometric function. Namely, for $|z|<1$, let $F(a, b, c, z)$ be the function given by

$$
F(a, b, c, z)=\sum_{n=0}^{\infty} \frac{(a)_{n}(b)_{n}}{(c)_{n}} \frac{z^{n}}{n !},
$$

where we write $(a)_{0}=1$ and $(a)_{n}=\Gamma(a+n) / \Gamma(a)$ for $n \geq 1$. For a vector $v \in V_{1}$ and a plane $z \in \mathbb{D}$, denote by $v_{z^{\perp}}$ the projection of $v$ to the orthogonal complement 
$z^{\perp}$ of $z$ in $V_{1}$. Then [Oda and Tsuzuki 2003, (2.5.3)]:

$\phi^{(2)}(v, z, s)=$

$$
-\frac{\Gamma\left(\frac{s+\rho_{0}}{2}\right) \Gamma\left(\frac{s-\rho_{0}}{2}+1\right)}{2 \Gamma(s+1)}\left(\frac{Q(v)}{Q\left(v_{z^{\perp}}\right)}\right)^{\frac{s+\rho_{0}}{2}} F\left(\frac{s+\rho_{0}}{2}, \frac{s-\rho_{0}}{2}+1, s+1, \frac{Q(v)}{Q\left(v_{z^{\perp}}\right)}\right) .
$$

3B. Green currents for special divisors. The functions $\phi^{(2)}(v, z, s)$ can be used to construct Green functions for the special divisors introduced above. Namely, let $\Gamma \subset H(\mathbb{R})$ be of the form $\Gamma=H_{+}(\mathbb{Q}) \cap K$ and $v \in V(F)$ be a vector of totally positive norm. Recall that we write $\Gamma_{v}=\Gamma \cap H_{v}(\mathbb{R})$. For $\operatorname{Re}(s)>\rho_{0}$, define

$$
G(v, z, s)_{\Gamma}=2 \sum_{\gamma \in \Gamma_{v} \backslash \Gamma} \phi^{(2)}(v, \gamma z, s)
$$

The sum converges absolutely a.e. and defines an integrable function $G(v, s)_{\Gamma}$ on $X_{\Gamma}$ [Oda and Tsuzuki 2003, Proposition 3.1.1]. Denote by $\left[G(v, s)_{\Gamma}\right]$ the associated current on $X_{\Gamma}$, defined by

$$
\left[G(v, s)_{\Gamma}\right](\alpha)=\int_{X_{\Gamma}} G(v, z, s)_{\Gamma} \cdot \alpha(z)
$$

for $\alpha \in \mathscr{A}_{c}^{2 n}\left(X_{\Gamma}\right)$. This current admits meromorphic continuation to $s \in \mathbb{C}$ with only simple poles [Oda and Tsuzuki 2003, Theorem 6.3.1]. In fact, as shown by Bruinier [2012, Theorem 5.12], one can refine this result to show that the function $G(v, z, s)_{\Gamma}$ itself has meromorphic continuation to the whole complex plane and that the resulting function is real analytic on $X_{\Gamma}-Z(v)_{\Gamma}$. Define

$$
G(v)_{\Gamma}=\mathrm{CT}_{s=\rho_{0}} G(v, s)_{\Gamma}
$$

to be the constant term of $G(v, s)_{\Gamma}$ at $s=\rho_{0}$.

Theorem 3.2 [Bruinier 2012, Theorem 5.14, Corollary 5.16]. The function $G(v)_{\Gamma}$ is real analytic on $X_{\Gamma}-Z(v)_{\Gamma}$ and has a logarithmic singularity on $Z(v)_{\Gamma}$. The form $d d^{c} G(v)_{\Gamma}=-(2 \pi i)^{-1} \partial \bar{\partial} G(v)_{\Gamma}$ extends to a $\mathscr{C}^{\infty}$ form on $X_{\Gamma}$ and one has the equation of currents:

$$
d d^{c}\left[G(v)_{\Gamma}\right]=\delta_{Z(v)_{\Gamma}}+\left[d d^{c} G(v)_{\Gamma}\right]
$$

Consider now a pair of vectors $v, w$ spanning a totally positive definite plane $U$ in $V$. Denote by $p_{v^{\perp}}(w)$ the projection of $w$ to the orthogonal complement of $v$. Recall that we write $X(v)_{\Gamma}=\Gamma_{v} \backslash \mathbb{D}_{v}^{+}$and $\Gamma_{v, w}=\Gamma \cap H_{U}(\mathbb{R})$. The map

$$
\Gamma_{v, w} \backslash \mathbb{D}_{U}^{+} \rightarrow X(v)_{\Gamma}
$$


then defines an effective divisor $Z(v, w)_{\Gamma}$ in $X(v)_{\Gamma}$. We define

$$
G(v, w, z, s)_{\Gamma}=2 \sum_{\gamma \in \Gamma_{v, w} \backslash \Gamma_{v}} \phi_{\mathbb{D}_{v}}^{(2)}\left(p_{v^{\perp}}(w), \gamma z, s\right) .
$$

The results described above imply that the sum converges when $\operatorname{Re}(s) \gg 0$ to an integrable function on $X(v)_{\Gamma}$, and that we have a meromorphic continuation property, so that we can define

$$
G(v, w, z)_{\Gamma}=\mathrm{CT}_{s=(n-1) / 2} G(v, w, z, s)_{\Gamma} .
$$

The function $G(v, w)_{\Gamma}$ is then real analytic on $X(v)_{\Gamma}-Z(v, w)_{\Gamma}$ and has a logarithmic singularity on $Z(v, w)_{\Gamma}$.

3C. The functions $\phi(v, w, z, s)$ on $\mathbb{D}$. For a pair of vectors $v, w \in V_{1}$, denote by $p_{w}(v)$ and $p_{w^{\perp}}(v)$ the projection of $v$ to the line spanned by $w$ and to the orthogonal complement of $w$, respectively.

Definition 3.3. Let $v, w$ be a pair of vectors in $V_{1}$ spanning a positive definite plane and let $s_{0}=(n-1) / 2$. For $\operatorname{Re}(s)>s_{0}$, define

$$
\begin{aligned}
\phi(v, w, z, s)=- & \frac{1}{2} \frac{\Gamma\left(\frac{s+s_{0}}{2}\right) \Gamma\left(\frac{s-s_{0}}{2}+1\right)}{\Gamma(s+1)}\left(\frac{Q(v)-Q\left(p_{w}(v)\right)}{Q\left(v_{z^{\perp}}\right)-Q\left(p_{w}(v)\right)}\right)^{\frac{s+s_{0}}{2}} \\
& \times F\left(\frac{s+s_{0}}{2}, \frac{s-s_{0}}{2}+1, s+1, \frac{Q(v)-Q\left(p_{w}(v)\right)}{Q\left(v_{z^{\perp}}\right)-Q\left(p_{w}(v)\right)}\right) .
\end{aligned}
$$

The following basic properties of $\phi(v, w, z, s)$ are easily checked.

Lemma 3.4. (1) For every $h \in H_{v}(\mathbb{R}), \phi(v, w, z, s)=\phi(v, w, h z, s)$.

(2) For every $h \in H(\mathbb{R}), \phi(h v, h w, h z, s)=\phi(v, w, z, s)$.

(3) The restriction of $\phi(v, w, z, s)$ to $\mathbb{D}_{w}$ equals $\phi_{\mathbb{D}_{w}}^{(2)}\left(p_{w^{\perp}}(v), z, s\right)$.

(4) Consider the function $\phi(v, w, g, s)=\phi\left(v, w, g z_{0}, s\right)$, for $g \in G$. It belongs to $\mathscr{C}^{\infty}\left(G-G_{v} K_{z_{0}}\right)$ and satisfies $\phi\left(v, w, g^{\prime} g k, s\right)=\phi(v, w, g, s)$ for every $g^{\prime} \in G_{v}, k \in K_{z_{0}}$. Writing $G=G_{v} A^{+} K_{z_{0}}$ as above, we have

$$
\begin{array}{ll}
\phi\left(v, w, a_{t}, s\right)=\log (t)+O(1) & \text { as } t \rightarrow 0, \\
\phi\left(v, w, a_{t}, s\right)=O\left(e^{-\left(\operatorname{Re}(s)+s_{0}\right) t}\right) & \text { as } t \rightarrow+\infty .
\end{array}
$$

Note that (1) and (2) imply $\phi(v, w, z, s)=\phi\left(v, h_{v} w, z, s\right)$ for every $h_{v} \in H_{v}(\mathbb{R})$, so that for fixed $v, z, s$, the function $\phi(v, w, z, s)$ only depends on the $H_{v}(\mathbb{R})$ orbit of $w$. Moreover, property (3-12) also holds for all partial derivatives of $\phi(v, w, z, s)$. Note also that property (3-11) implies that $\phi(v, w, z, s)$ is locally integrable. Concerning the behavior of the partial derivatives of $\phi(v, w, z, s)$ as $z$ approaches $\mathbb{D}_{v}$, we have the following lemma. 
Lemma 3.5. Each of the partial derivatives $\partial \phi(v, w, z, s), \bar{\partial} \phi(v, w, z, s)$ and $\partial \bar{\partial} \phi(v, w, z, s)$ is locally integrable.

Proof. Let $U \subset \mathbb{D}^{+}$be open with coordinates $\left\{z_{1}, \ldots, z_{n}\right\}$ such that the analytic divisor $\mathbb{D}_{v}^{+} \cap U$ is given by the equation $z_{1}=0$ on $U$. Choosing a trivialization of $\mathscr{L}$ on $U$ we can write $-Q\left(v_{z}\right)=\left\|s_{v}(z)\right\|^{2}=h(z)\left|z_{1}\right|^{2}$, where $h(z)$ is real analytic on $U$. It follows from the expansion of the hypergeometric function $F(a, b, a+b, w)$ around $w=1$ (see [Lebedev 1965, (9.7.5)]) that, for fixed $v, w, s$ and $z \in U$,

$$
\phi(v, w, z, s)=\log \left|z_{1}\right|+\left|z_{1}\right|^{2} \log \left|z_{1}\right| f(z)+g(z),
$$

where $f$ and $g$ are real analytic functions on $U$. Thus at worst the singularities of $\|\partial \phi(v, w, z, s)\|,\|\bar{\partial} \phi(v, w, z, s)\|$ and $\|\partial \bar{\partial} \phi(v, w, z, s)\|$ are of the form $\left|z_{1}\right|^{-1}$ or $\log \left|z_{1}\right|$, and the statement follows.

The function $\phi(v, w, z, s)$ can also be obtained as a Laplace transform of a certain Whittaker function that depends on $s$. Namely, consider Kummer's hypergeometric function:

$$
M(a, b, z)=\sum_{n=0}^{+\infty} \frac{(a)_{n}}{(b)_{n}} \frac{z^{n}}{n !} .
$$

The function

$$
M_{v, \mu}(z)=e^{-z / 2} z^{1 / 2+\mu} M\left(\frac{1}{2}+\mu-v, 1+2 \mu, z\right)
$$

is then a solution of the Whittaker differential equation

$$
\frac{d^{2} w}{d z^{2}}+\left(-\frac{1}{4}+\frac{v}{z}-\frac{\mu^{2}-1 / 4}{z^{2}}\right) w=0
$$

It is characterized among solutions of this equation by its asymptotic behavior, given by:

$$
\begin{array}{ll}
M_{v, \mu}(z)=z^{\mu+1 / 2}(1+O(z)) & \text { when } z \rightarrow 0, \\
M_{v, \mu}(z)=\frac{\Gamma(1+2 \mu)}{\Gamma(\mu-v+1 / 2)} e^{z / 2} z^{-v}\left(1+O\left(z^{-1}\right)\right) & \text { when } z \rightarrow \infty .
\end{array}
$$

For a positive definite symmetric matrix $T=\left(\begin{array}{ll}a & b \\ b & c\end{array}\right)$, define

$$
\begin{gathered}
s_{0}=(n-1) / 2, \quad k=1-s_{0}, \\
C(T, s)=-\frac{1}{2} \frac{\Gamma\left(\frac{s-s_{0}}{2}+1\right)}{\Gamma(s+1)}\left(\frac{4 \pi \operatorname{det} T}{c}\right)^{-k / 2},
\end{gathered}
$$

and

$$
M_{T}(y, s)=C(T, s)|y|^{-k / 2} M_{-k / 2, s / 2}\left(\left|\frac{4 \pi \operatorname{det} T}{c} y\right|\right) e^{\frac{2 \pi b^{2}}{c} y}, \quad \operatorname{Re}(s)>s_{0} .
$$


Now consider $v, w \in V_{1}$ spanning a positive definite plane and denote by

$$
T(v, w)=\frac{1}{2}\left(\begin{array}{ll}
(v, v) & (v, w) \\
(v, w) & (w, w)
\end{array}\right)
$$

the associated moment matrix. Then (see [Erdélyi et al. 1954, p. 215, §4.22 (11)])

$$
\phi(v, w, z, s)=\int_{0}^{\infty} M_{T(v, w)}(y, s) e^{-2 \pi y\left(Q\left(v_{z^{\perp}}\right)-Q\left(v_{z}\right)\right)} \frac{d y}{y} .
$$

3D. Currents in $\mathscr{D}^{1,1}\left(X_{\Gamma}\right)$. We now define some $(1,1)$-forms and currents on $X_{\Gamma}$ by summation over translates by elements of $\Gamma$ of some differential forms with singularities on $\mathbb{D}$. For vectors $v, w \in V(F)$ spanning a totally positive definite space, consider the $(1,1)$-form $\omega(v, w, z, s)$ defined for $z \in \mathbb{D}^{+}-\left(\mathbb{D}_{v}^{+} \cup \mathbb{D}_{w}^{+}\right)$by

$$
\begin{aligned}
\omega(v, w, z, s)= & \bar{\partial}(\phi(w, v, z, s) \partial \phi(v, w, z, s)) \\
= & \bar{\partial} \phi(w, v, z, s) \wedge \partial \phi(v, w, z, s) \\
& +\phi(w, v, z, s) \bar{\partial} \partial \phi(v, w, z, s) .
\end{aligned}
$$

We would like to define a $(1,1)$-form on $X_{\Gamma}$ by averaging the form $\omega(v, w, z, s)$ over $\Gamma$. Before making such a definition, we need to check that the resulting sums converge in a suitable sense. This is the content of the next result. Note that we have

$$
\gamma^{*}(\omega(v, w, s))(z)=\omega\left(\gamma^{-1} v, \gamma^{-1} w, z, s\right)
$$

for all $\gamma \in \Gamma$, due to the invariance property in Lemma 3.4(2).

Proposition 3.6. Let $v, w \in V(F)$ be vectors spanning a totally positive definite plane. Let $U=\mathbb{D}^{+}-\left(\Gamma \cdot \mathbb{D}_{v}^{+} \cup \Gamma \cdot \mathbb{D}_{w}^{+}\right)$. For $\operatorname{Re}(s) \gg 0$, the sum

$$
\sum_{\gamma \in \Gamma_{v, w} \backslash \Gamma} \omega\left(\gamma^{-1} v, \gamma^{-1} w, z, s\right)
$$

and all its partial derivatives converge normally for every $z \in U$.

Proof. Since the function $\phi(v, w, \gamma z, s)$ is defined and smooth for every $z \in$ $\mathbb{D}-\mathbb{D}_{\gamma^{-1} v}$, all the terms in the sum are defined whenever $z \in U$. Fix $z_{0} \in U$ and let $U_{0} \subset U$ be a compact neighborhood of $z_{0}$; then there exists $\epsilon>0$ such that $\left|Q\left((\gamma v)_{z}\right)\right|>\epsilon$ and $\left|Q\left((\gamma w)_{z}\right)\right|>\epsilon$ for all $\gamma \in \Gamma$ and all $z \in U_{0}$. It follows from Lemma 3.4 that on $U_{0}$ we have

$$
\left\|\omega\left(\gamma^{-1} v, \gamma^{-1} w, z, s\right)\right\|<C_{\epsilon}\left|Q\left(\left(\gamma^{-1} v\right)_{z^{\perp}}\right)\right|^{-\left(s+s_{0}\right) / 2}\left|Q\left(\left(\gamma^{-1} w\right)_{z^{\perp}}\right)\right|^{-\left(s+s_{0}\right) / 2}
$$

for some constant $C_{\epsilon}>0$, and a similar bound holds for the sums of all the partial derivatives of the summands. Thus, for $z \in U_{0}$, the sums in the statement are 
dominated by a constant multiple of

$$
\sum_{\gamma \in \Gamma_{v, w} \backslash \Gamma}\left|Q\left(\left(\gamma^{-1} v\right)_{z^{\perp}}\right)\right|^{-\left(s+s_{0}\right) / 2}\left|Q\left(\left(\gamma^{-1} w\right)_{z^{\perp}}\right)\right|^{-\left(s+s_{0}\right) / 2} .
$$

Pick a lattice $L \subset V(F)$ such that $\Gamma \cdot(v, w) \subset L^{2}$; then the above sum is dominated by

$$
\left(\sum_{\substack{\lambda \in L \\ Q(\lambda)=Q(v)}}\left|Q\left(\lambda_{z^{\perp}}\right)\right|^{-\left(s+s_{0}\right) / 2}\right)\left(\sum_{\substack{\lambda \in L \\ Q(\lambda)=Q(w)}}\left|Q\left(\lambda_{z^{\perp}}\right)\right|^{-\left(s+s_{0}\right) / 2}\right),
$$

which converges normally on $U$, since the assignment $v \mapsto Q\left(v_{z^{\perp}}\right)-Q\left(v_{z}\right)$ defines a positive definite quadratic form on $V_{1}$ that depends continuously on $z$.

Define

$$
\Phi(v, w, z, s)_{\Gamma}=2 \sum_{\gamma \in \Gamma_{v, w} \backslash \Gamma} \omega\left(\gamma^{-1} v, \gamma^{-1} w, z, s\right),
$$

and note that

$$
\Phi(v, w, z, s)_{\Gamma}=\Phi(\gamma v, \gamma w, z, s)_{\Gamma} \quad \text { for all } \gamma \in \Gamma .
$$

Proposition 3.6 shows that $\Phi(v, w, \cdot, s)_{\Gamma}$ converges and defines a smooth $(1,1)$-form on $X_{\Gamma}-\left(Z(v)_{\Gamma} \cup Z(w)_{\Gamma}\right)$.

Denote the cotangent bundle of a manifold $X$ by $T^{*} X$. A section $s$ of a metrized vector bundle $(E,\|\cdot\|)$ over a manifold $X$ endowed with a measure $d \mu(z)$ is said to be $L^{1}$ (or integrable) if $\|s\| \in L^{1}(X, d \mu(z))$. Our next goal is to show that $\Phi(v, w, z, s)_{\Gamma}$ is integrable on $X_{\Gamma}$; this is the content of Proposition 3.9. The next two lemmas will be used in the proof.

Lemma 3.7. Let $M$ be a complete, simply connected Riemannian manifold of everywhere nonpositive sectional curvature. Let $X, Y \subset M$ be complete, simply connected, totally geodesic submanifolds that intersect transversely and at a single point $z_{0} \in M$. For $z \in M$, denote by $d\left(z, z_{0}\right)$ the geodesic distance between $z$ and $z_{0}$ and by $d_{X}(z)$ and $d_{Y}(z)$ the geodesic distance from $z$ to $X$ and from $z$ to $Y$, respectively. Then there exists a constant $k>0$ such that $d\left(z_{0}, z\right) \geq t$ implies $\max \left\{d_{X}(z), d_{Y}(z)\right\} \geq k t$ for every $t \geq 0$.

Proof. Let $d>0$ and suppose that $\max \left\{d_{X}(z), d_{Y}(z)\right\}<d$. Choose points $z_{X} \in X$ and $z_{Y} \in Y$ such that $d\left(z_{X}, z\right)<d$ and $d\left(z_{Y}, z\right)<d$. Let $\gamma\left(z_{X}, z_{Y}\right)$ be the geodesic segment connecting $z_{X}$ and $z_{Y}$; such a geodesic exists, is unique and minimizes the distance (see [Chavel 2006, Exercise IV.12(a)]), hence its length $l\left(\gamma\left(z_{X}, z_{Y}\right)\right)$ satisfies $l\left(\gamma\left(z_{X}, z_{Y}\right)\right)<2 d$. Let $\gamma\left(z_{0}, z_{X}\right)$ and $\gamma\left(z_{0}, z_{Y}\right)$ be the geodesic segments in $X$ and $Y$ connecting $z_{0}$ and $z_{X}$ and $z_{0}$ and $z_{Y}$, respectively; as before, these geodesics exist and are unique and minimizing. 
Consider now the triangle $T$ in $M$ with sides $\left\{\gamma\left(z_{0}, z_{X}\right), \gamma\left(z_{0}, z_{Y}\right), \gamma\left(z_{X}, z_{Y}\right)\right\}$. This is a geodesic triangle since $X$ and $Y$ are totally geodesic. Note that the angle at $z_{0}$ is bounded below since $X$ and $Y$ are assumed to intersect transversely. By the Cartan-Hadamard theorem (see [Bridson and Haefliger 1999, Theorem II.4.1]), the space $M$ is a $C A T(0)$ space, in other words the (unique up to congruence) triangle in the euclidean plane with same sides as $T$ has larger angles than $T$ (see [Bridson and Haefliger 1999, Proposition II.1.7(4)]). It follows that $d\left(z_{0}, z_{X}\right) \leq c d\left(z_{X}, z_{Y}\right)$ for some positive constant $c$. Hence $d\left(z_{0}, z\right) \leq d\left(z_{0}, z_{X}\right)+d\left(z_{X}, z\right)<(2 c+1) d$ as required.

Lemma 3.8. Let $M, X, Y$ be as in Lemma 3.7. Assume that the codimension of $X$ and $Y$ in $M$ is greater than one and that the sectional curvature of $M$ is bounded below. Let $f_{1, s}, f_{2, s}: \mathbb{R}_{>0} \rightarrow \mathbb{R}_{>0}$ be continuous functions defined for $\operatorname{Re}(s)>s_{0}>0$ such that

$$
\begin{aligned}
t f_{i, s}(t) & =O(1), \quad \text { as } t \rightarrow 0, \\
f_{i, s}(t) & =e^{-\operatorname{Re}(s) t}, \quad \text { as } t \rightarrow \infty,
\end{aligned}
$$

for $i=1,2$. Let $d \mu(z)$ be the Riemannian volume element of $M$. Then, with notation as in Lemma 3.7, we have

$$
\int_{M} f_{1, s}\left(d_{X}(z)\right) f_{2, s}\left(d_{Y}(z)\right) d \mu(z)<\infty
$$

for $\operatorname{Re}(s) \gg 0$.

Proof. Let $U_{X}=\left\{z \in M \mid d_{X}(z) \leq 1\right\}$ and $U_{Y}=\left\{z \in M \mid d_{Y}(z) \leq 1\right\}$ be tubular neighborhoods around $X$ and $Y$ of radius 1 . Let $U=M-\left(U_{X} \cup U_{Y}\right)$. It suffices to show that $f_{s}(z)=f_{1, s}\left(d_{X}(z)\right) f_{2, s}\left(d_{Y}(z)\right)$ is integrable when restricted to $U, U_{X}$ and $U_{Y}$.

Consider first the integral over $U$. By hypothesis, the functions $f_{1, s}\left(d_{X}(z)\right)$ and $f_{2, s}\left(d_{Y}(z)\right)$ are bounded on $U$. By Lemma 3.7, there exists a constant $k>0$ such that

$$
f_{s}(z)=O\left(e^{-\operatorname{Re}(s) k d\left(z, z_{0}\right)}\right)
$$

for $z \in U$. Let $S\left(z_{0}, t\right)$ be the geodesic sphere with center $z_{0}$ and radius $t$ and denote by $A(t)$ its area. Since $M$ has curvature that is bounded below, there exists $\rho>0$ such that $A(t)=O\left(e^{\rho t}\right)$ (see [Chavel 2006, Theorem III.4.4]). It follows that

$$
\int_{U} f_{s}(z) d \mu(z)<\infty
$$

whenever $\operatorname{Re}(s)>\rho / k$.

Now consider the integral over $U_{X}$ (the same argument works for $U_{Y}$ ). Since $f_{s}(z)$ is locally integrable, it suffices to integrate over $U_{X}-\left(U_{X} \cap U_{Y}\right)$. The inclusion $i: X \subset U_{X}$ admits a left inverse $\pi: U_{X} \rightarrow X$ whose fibers are diffeomorphic to 
the closed unit disk in $\mathbb{C}$ (this is because the exponential map from the total space of the normal bundle of $X$ to $M$ is a diffeomorphism). We can compute the integral over $U_{X}$ by first integrating over the fibers of $\pi$ and then integrating over $X$. By hypothesis, the integral of $f_{s}(z)$ over $\pi^{-1}(z)$ is $O\left(e^{-\operatorname{Re}(s) d\left(z, z_{0}\right)}\right)$ for every $z \in X-\left(X \cap U_{Y}\right)$. Now the resulting integral over $X$ converges for $\operatorname{Re}(s) \gg 0$ since the area of a sphere of radius $t$ in $X$ is $O\left(e^{\rho t}\right)$ as above.

We can now prove that $\Phi(v, w, z, s)_{\Gamma}$ is integrable on $X_{\Gamma}$. Recall that $\mathbb{D}$ carries an $H(\mathbb{R})$-invariant Riemannian metric; it induces an invariant metric on $\wedge^{2} T^{*} \mathbb{D}$ that we denote by $\|\cdot\|$.

Proposition 3.9. Let $v, w \in V(F)$ be vectors spanning a totally positive plane. For $\operatorname{Re}(s) \gg 0$, the sum $\Phi(v, w, z, s)_{\Gamma}$ converges outside a set of measure zero in $X_{\Gamma}$ and defines an $L^{1}$ section of $\left(\bigwedge^{2} T^{*} X_{\Gamma},\|\cdot\|\right)$.

Proof. The sum converges for $z \notin Z(v)_{\Gamma} \cup Z(w)_{\Gamma}$ by Proposition 3.6, and this set has measure zero. Thus it remains to prove integrability. We need to show that

$$
\int_{X_{\Gamma}}\|\Phi(v, w, z, s)\| d \mu(z)
$$

is convergent, where $d \mu(z)$ denotes an invariant volume form on $\mathbb{D}^{+}$. By Fubini's theorem, it suffices to show that

$$
\int_{\Gamma_{v, w} \backslash \mathbb{D}^{+}}\|\omega(w, v, z, s)\| d \mu(z)<\infty .
$$

Let $H^{\prime}(\mathbb{R})=\left(H_{v}\right)_{+}(\mathbb{R}) \cap\left(H_{w}\right)_{+}(\mathbb{R})$ and let $Z_{H^{\prime}}(\mathbb{R})$ be the center of $H^{\prime}(\mathbb{R})$. Since the integrand is left invariant under $H^{\prime}(\mathbb{R})$ by Lemma 3.4 and $Z_{H^{\prime}}(\mathbb{R}) \Gamma_{v, w} \backslash H^{\prime}(\mathbb{R})$ has finite volume (see [Borel 1969]), this is equivalent to

$$
\int_{H^{\prime}(\mathbb{R}) \backslash \mathbb{D}^{+}}\|\omega(w, v, z, s)\| d \mu(z)<\infty .
$$

We now apply Lemma 3.8. Namely, let $M=H^{\prime}(\mathbb{R}) \backslash \mathbb{D}^{+}$. Let $X=H^{\prime}(\mathbb{R}) \backslash \mathbb{D}_{v}^{+}$and $Y=H^{\prime}(\mathbb{R}) \backslash \mathbb{D}_{w}^{+}$. Note that there is a map $\pi: \mathbb{D}^{+} \rightarrow \mathbb{D}_{v}^{+}$that is left inverse to the inclusion $\mathbb{D}_{v}^{+} \subset \mathbb{D}^{+}$and turns $\mathbb{D}^{+}$into an $H_{v}(\mathbb{R})_{+}$-equivariant real vector bundle of rank 2 over $\mathbb{D}_{v}^{+}$(see [Kudla and Millson 1988, p. 26]). Hence the inclusions

$$
\{*\}=H^{\prime}(\mathbb{R}) \backslash \mathbb{D}_{v, w}^{+} \subset H^{\prime}(\mathbb{R}) \backslash \mathbb{D}_{v}^{+} \subset H^{\prime}(\mathbb{R}) \backslash \mathbb{D}^{+}
$$

are diffeomorphic to zero sections of vector bundles, in particular they are simply connected. Moreover $\mathbb{D}_{v}^{+}$and $\mathbb{D}_{w}^{+}$are totally geodesic submanifolds of $\mathbb{D}^{+}$, and the latter is known to have sectional curvatures that are bounded below and everywhere nonpositive. Hence $X, Y$ and $M$ satisfy the hypotheses in Lemma 3.7 and Lemma 3.8. Moreover, by Lemma 3.5, the integrand also satisfies the hypotheses in Lemma 3.8; applying it gives $(*)$ and hence the assertion. 
Since $\Phi(v, w, z, s)_{\Gamma}$ is an integrable section of $\bigwedge^{2} T^{*} X_{\Gamma}$, its coordinates in any chart $U \subset X_{\Gamma}$ are locally integrable functions. Thus $\Phi(v, w, z, s)_{\Gamma}$ defines a current on $X_{\Gamma}$.

Definition 3.10. Let $v, w \in V(F)$ be vectors spanning a totally positive definite plane. For $\operatorname{Re}(s) \gg 0$, define a current $\left[\Phi(v, w, s)_{\Gamma}\right] \in \mathscr{D}^{1,1}\left(X_{\Gamma}\right)$ by

$$
\left[\Phi(v, w, s)_{\Gamma}\right](\omega)=\int_{X_{\Gamma}} \Phi(v, w, s)_{\Gamma} \wedge \omega
$$

for $\omega \in \mathscr{A}_{c}^{n-1, n-1}\left(X_{\Gamma}\right)$.

Recall that we assume $\Gamma=H_{+}(\mathbb{Q}) \cap K$ for some open compact $K \subset H\left(\mathbb{A}_{f}\right)$. For $h \in H\left(\mathbb{A}_{f}\right)$, we write $\Gamma_{h}=H_{+}(\mathbb{Q}) \cap h K h^{-1}$ and we define

$$
\Phi(v, w, h, s)_{\Gamma}=\Phi(v, w, s)_{\Gamma_{h}},
$$

an $L^{1}$ section of $\wedge^{2} T^{*}\left(\Gamma_{h} \backslash \mathbb{D}^{+}\right)$. As above, we denote by $\left[\Phi(v, w, h, s)_{\Gamma}\right]$ the associated current in $\mathscr{D}^{1,1}\left(\Gamma_{h} \backslash \mathbb{D}^{+}\right)$.

3E. Some properties of $\left[\Phi(v, w, h, s)_{\Gamma}\right]$. We now introduce another family of currents $\left[\Phi(v, w)_{\Gamma}\right]$ on $X_{\Gamma}$. These currents are obtained by restricting a compactly supported form $\omega \in \mathscr{A}_{c}^{n-1, n-1}\left(X_{\Gamma}\right)$ to a special divisor $X(v)_{\Gamma}$ and integrating it against a Green function of the form (3-6). In this section we will prove that the current $\left[\Phi(v, w, s)_{\Gamma}\right]$ introduced above, regarded modulo $\operatorname{Im}(\partial)+\operatorname{Im}(\bar{\partial})$, admits meromorphic continuation to the complex plane $s$ and that the current $\left[\Phi(v, w)_{\Gamma}\right]$ is cohomologous to the current obtained as the constant term of the meromorphic continuation of $\left[\Phi(v, w, s)_{\Gamma}\right]$ at a certain value $s=s_{0}$.

For $v \in V(F)$ of totally positive norm, denote by $\delta_{X(v)_{\Gamma}} \in \mathscr{D}^{1,1}\left(X_{\Gamma}\right)$ the current of integration along $X(v)_{\Gamma}$. That is, for $\omega \in \mathscr{A}_{c}^{n-1, n-1}\left(X_{\Gamma}\right)$, we have

$$
\delta_{X(v)_{\Gamma}}(\omega)=\int_{X(v)_{\Gamma}} \omega .
$$

Consider now $v, w \in V(F)$ spanning a totally positive definite plane. In Section 3B we recalled the construction (see [Bruinier 2012; Oda and Tsuzuki 2003]) of a function $G(v, w)_{\Gamma} \in \mathscr{C}^{\infty}\left(X(v)_{\Gamma}-Z(v, w)_{\Gamma}\right)$. The function has a logarithmic singularity along $Z(v, w)_{\Gamma}$, hence is locally integrable on $X(v)_{\Gamma}$ and defines an element of $\mathscr{D}^{0}\left(X(v)_{\Gamma}\right)$ that we denote by $\left[G(v, w)_{\Gamma}\right]$. Recall that there is a pushforward map

$$
f_{*}: \mathscr{D}^{0}\left(X(v)_{\Gamma}\right) \rightarrow \mathscr{D}^{1,1}\left(X_{\Gamma}\right)
$$

induced by $f: X(v)_{\Gamma} \rightarrow X_{\Gamma}$ and defined by $\left(f_{*}(\alpha), \omega\right)=\left(\alpha, f^{*}(\omega)\right)$ for $\alpha$ in $\mathscr{D}^{0}\left(X(v)_{\Gamma}\right)$ and $\omega$ in $\mathscr{A}_{c}^{n-1, n-1}\left(X_{\Gamma}\right)$. 
Definition 3.11. Let $v, w \in V(F)$ spanning a totally positive definite plane. Define the current $\left[\Phi(v, w)_{\Gamma}\right] \in \mathscr{D}^{1,1}\left(X_{\Gamma}\right)$ by

$$
\left[\Phi(v, w)_{\Gamma}\right]=2 \pi i \cdot f_{*}\left(\left[G(v, w)_{\Gamma}\right]\right) .
$$

For $h \in H\left(\mathbb{A}_{f}\right)$ and $K \subset H\left(\mathbb{A}_{f}\right)$ such that $\Gamma=H_{+}(\mathbb{Q}) \cap K$, define

$$
\left[\Phi(v, w, h)_{\Gamma}\right]=\left[\Phi(v, w)_{\Gamma_{h}}\right],
$$

where $\Gamma_{h}=H_{+}(\mathbb{Q}) \cap h K h^{-1}$.

That is, for $\omega \in \mathscr{A}_{c}^{n-1, n-1}\left(X_{\Gamma}\right)$, we have

$$
\left[\Phi(v, w)_{\Gamma}\right](\omega)=2 \pi i \int_{X(v)_{\Gamma}} G(v, w)_{\Gamma} \cdot \omega .
$$

See Section 4B2 for an example.

The next proposition relates the currents $\left[\Phi(v, w)_{\Gamma}\right]$ and $\left[\Phi(v, w, s)_{\Gamma}\right]$ and is key to the computation of values of $\left[\Phi(v, w)_{\Gamma}\right]$ on forms obtained as theta lifts as below. Let

$$
\tilde{\mathscr{D}}^{1,1}\left(X_{\Gamma}\right)=\mathscr{D}^{1,1}\left(X_{\Gamma}\right) /(\operatorname{Im}(\partial)+\operatorname{Im}(\bar{\partial})) .
$$

We let $\left[\Phi(v, w, s)_{\Gamma]}\right]$ and $\left[\Phi(v, w)_{\Gamma}\right]$ also denote the classes of $\left[\Phi(v, w, s)_{\Gamma}\right]$ and $\left[\Phi(v, w)_{\Gamma}\right]$ in $\tilde{D}^{1,1}\left(X_{\Gamma}\right)$.

Proposition 3.12. The current $\left[\Phi(v, w, s)_{\Gamma}\right] \in \tilde{\mathscr{D}}^{1,1}\left(X_{\Gamma}\right)$ admits meromorphic continuation to $s \in \mathbb{C}$. Let $\mathrm{CT}_{s=s_{0}}\left[\Phi(v, w, s)_{\Gamma}\right] \in \tilde{\mathscr{D}}^{1,1}\left(X_{\Gamma}\right)$ denote the constant term of $\left[\Phi(v, w, s)_{\Gamma}\right]$ at $s=s_{0}$. Then

$$
\mathrm{CT}_{s=s_{0}}\left[\Phi(v, w, s)_{\Gamma}\right]=\left[\Phi(v, w)_{\Gamma}\right]
$$

as elements of $\tilde{\mathscr{D}}^{1,1}\left(X_{\Gamma}\right)$.

Proof. Let $\alpha \in \mathscr{A}_{c}^{n-1, n-1}\left(X_{\Gamma}\right)$. By Proposition 3.9, we have

$$
\left[\Phi(v, w, s)_{\Gamma}\right](\alpha)=2 \int_{\Gamma_{v, w} \backslash \mathbb{D}^{+}} \omega(v, w, z, s) \wedge \alpha(z) .
$$

For fixed $s$, write $g_{v}(z)=\phi(v, w, z, s)$ and $g_{w}(z)=\phi(w, v, z, s)$. We regard $g_{v}$ as a smooth function defined on $\Gamma_{v, w} \backslash \mathbb{D}^{+}-\Gamma_{v, w} \backslash \mathbb{D}_{v}^{+}$. If we choose an open $U \subset \Gamma_{v, w} \backslash \mathbb{D}^{+}$such that the analytic divisor $\left(\Gamma_{v, w} \backslash \mathbb{D}_{v}^{+}\right) \cap U$ is given by the equation $z=0$, then it follows from (3-13) that

$$
\partial g_{v}(z)=\frac{d z}{z}+o\left(|z|^{-1}\right), \quad \bar{\partial} g_{v}(z)=\frac{d \bar{z}}{\bar{z}}+o\left(|z|^{-1}\right) .
$$

(Here $o\left(|z|^{-1}\right)$ stands for a differential form $\alpha$ on $U-\left(\Gamma_{v, w} \backslash \mathbb{D}_{v}^{+}\right) \cap U$ such that the components of $|z| \alpha$ extend to continuous functions on $U$ vanishing on $\left(\Gamma_{v, w} \backslash \mathbb{D}_{v}^{+}\right) \cap U$.) Similar statements hold for $g_{w}(z)$ when $z$ approaches $\Gamma_{v, w} \backslash \mathbb{D}_{w}^{+}$. Denote by $\delta_{v} \in \mathscr{D}^{1,1}\left(\Gamma_{v, w} \backslash \mathbb{D}^{+}\right)$the current given by integration on $\Gamma_{v, w} \backslash \mathbb{D}_{v}^{+}$. The 
following identity of currents on $\Gamma_{v, w} \backslash \mathbb{D}^{+}$follows from Stokes's theorem applied to $\Gamma_{v, w} \backslash \mathbb{D}^{+}-\left(\Gamma_{v, w} \backslash \mathbb{D}_{v}^{+} \cup \Gamma_{v, w} \backslash \mathbb{D}_{w}^{+}\right)$:

$$
\bar{\partial}\left[g_{w} \partial g_{v}\right]=\left[\bar{\partial} g_{w} \partial g_{v}\right]+\left[g_{w} \bar{\partial} \partial g_{v}\right]-2 \pi i g_{w} \delta_{v} .
$$

We find that for any closed compactly supported form $\alpha_{c} \in \mathscr{A}_{c}^{n-1, n-1}\left(\Gamma_{v, w} \backslash \mathbb{D}^{+}\right)$,

$$
\int_{\Gamma_{v, w} \backslash \mathbb{D}^{+}} \omega(v, w, z, s) \wedge \alpha_{c}(z)=2 \pi i \int_{\Gamma_{v, w} \backslash \mathbb{D}_{v}^{+}} \phi(w, v, z, s) \alpha_{c}(z) .
$$

The form $\alpha(z)$ is not compactly supported, but we claim that (3-37) is still true for $\operatorname{Re}(s) \gg 0$ when we replace $\alpha_{c}(z)$ by $\alpha(z)$. Assuming this for now and using that the restriction of $\phi(w, v, z, s)$ to $\mathbb{D}_{v}$ equals $\phi_{\mathbb{D}_{v}}^{(2)}\left(p_{v^{\perp}}(w), z, s\right)$, we conclude that for $\operatorname{Re}(s) \gg 0$

$$
\begin{aligned}
{\left[\Phi(v, w, s)_{\Gamma}\right](\alpha) } & \equiv 2 \pi i \cdot 2 \int_{\Gamma_{v, w} \backslash \mathbb{D}_{v}^{+}} \phi_{\mathbb{D}_{v}}^{(2)}\left(p_{v^{\perp}}(w), z, s\right) \alpha(z) \\
& =2 \pi i \int_{\Gamma_{v} \backslash \mathbb{D}_{v}^{+}} 2 \sum_{\gamma \in \Gamma_{v, w} \backslash \Gamma_{v}} \phi_{\mathbb{D}_{v}}^{(2)}\left(p_{v^{\perp}}(w), \gamma z, s\right) \alpha(z) \\
& =2 \pi i \int_{\Gamma_{v} \backslash \mathbb{D}_{v}^{+}} G\left(p_{v^{\perp}}(w), z, s\right)_{\Gamma_{v}} \cdot \alpha(z) .
\end{aligned}
$$

This last equation defines a current on $X_{\Gamma}$ that admits meromorphic continuation to $s \in \mathbb{C}$ and whose constant term at $s=s_{0}$ is given by $\left[\Phi(v, w)_{\Gamma}\right]$; the claim follows from this.

It only remains to show that (3-37) still holds when we replace $\alpha_{c}(z)$ by $\alpha(z)$. Let $X=\Gamma_{v, w} \backslash \mathbb{D}^{+}$and consider the submanifolds $X_{v}=\Gamma_{v, w} \backslash \mathbb{D}_{v}^{+}$and $X_{w}=\Gamma_{v, w} \backslash \mathbb{D}_{w}^{+}$ of $X$. Let $X_{v, w}=X_{v} \cap X_{w}=\Gamma_{v, w} \backslash \mathbb{D}_{v, w}^{+}$. As remarked by Kudla and Millson [1988, p. 26], the exponential map of the normal bundle of $X_{v, w} \subset X$ is a diffeomorphism, and hence $X$ carries a natural vector bundle structure $\pi: X \rightarrow X_{v, w}$ of rank 4 over $X_{v, w}$ with totally geodesic fibers. For $t>0$, let $X_{v}(t)=\left\{z \in X \mid d_{X_{v}}(z) \leq t\right\}$ be the tubular neighborhood of radius $t$ around $X_{v}$; here $d_{X_{v}}(z)$ denotes the geodesic distance between $z$ and $X_{v}$. Define $X_{w}(t)$ and $X_{v, w}(t)$ similarly and let $X(t)=$ $X_{v, w}(t)-\left(X_{v}(1 / t) \cup X_{w}(1 / t)\right)$. Then we have $X-\left(X_{v} \cup X_{w}\right)=\bigcup_{t \geq 1} X(t)$ and

$$
\int_{X} \omega(v, w, z, s) \wedge \alpha=\lim _{t \rightarrow \infty} \int_{X(t)} \omega(v, w, z, s) \wedge \alpha .
$$

Denote by $S_{v, w}(t)=\partial X_{v, w}(t)$ the boundary of $X_{v, w}(t)$. By Stokes's theorem, (3-37) is equivalent to

$$
\int_{S_{v, w}(t)-\left(X_{v}(1 / t) \cup X_{w}(1 / t)\right)} \phi(w, v, z, s) \partial \phi(v, w, z, s) \wedge \alpha \longrightarrow 0,
$$


as $t \rightarrow \infty$. Since $\|\alpha\|$ is bounded, it suffices to show that

$$
\int_{S_{v, w}(t)-\left(X_{v}(1 / t) \cup X_{w}(1 / t)\right)}|\phi(w, v, z, s)| \cdot\|\partial \phi(v, w, z, s)\| d \mu(z) \longrightarrow 0,
$$

as $t \rightarrow \infty$. Now let $H^{\prime}(\mathbb{R})=\left(H_{v}\right)_{+}(\mathbb{R}) \cap\left(H_{w}\right)_{+}(\mathbb{R})$ and note that the integrand is invariant under $H^{\prime}(\mathbb{R})$. Let $M=H^{\prime}(\mathbb{R}) \backslash \mathbb{D}^{+}$and consider the submanifolds $X=H^{\prime}(\mathbb{R}) \backslash \mathbb{D}_{v}^{+}$and $Y=H^{\prime}(\mathbb{R}) \backslash \mathbb{D}_{w}^{+}$of $M$, whose intersection is a single point $z_{0}$. Let $S\left(z_{0}, t\right)$ be the sphere of geodesic radius $t$ around $z_{0}$ and let $X(1 / t)$ and $Y(1 / t)$ be tubular neighborhoods of $X$ and $Y$ with radius $1 / t$. Since $X_{v, w}$ has finite volume by [Borel 1969, Theorem 15.5], to show that the integrals in (3-38) tend to 0 it suffices to show that

$$
\int_{S\left(z_{0}, t\right)-(X(1 / t) \cup Y(1 / t))}|\phi(w, v, z, s)| \cdot\|\partial \phi(v, w, z, s)\| d \mu(z) \longrightarrow 0,
$$

as $t \rightarrow \infty$. Now Lemma 3.7 and Lemma 3.4 show that the integrand is $O\left(t e^{-k \operatorname{Re}(s) t}\right)$ for some positive constant $k>0$. Since the sectional curvatures of $M$ are bounded below, we have $\operatorname{Area}\left(S\left(z_{0}, t\right)\right)=O\left(e^{\rho t}\right)$ for some positive constant $\rho>0$ and hence (3-37) holds, with $\alpha_{c}$ replaced by $\alpha$, for $\operatorname{Re}(s)>\rho / k$.

3F. Currents on $\boldsymbol{X}_{\boldsymbol{K}}$. We introduce now currents in $\mathscr{D}^{1,1}\left(X_{K}\right)$. Fix a neat open compact subgroup $K \subset H\left(\mathbb{A}_{f}\right)$ and recall that we write

$$
X_{K}=H(\mathbb{Q}) \backslash\left(\mathbb{D} \times H\left(\mathbb{A}_{f}\right)\right) / K .
$$

Thus $X_{K}$ is a compact complex manifold with finitely many components. These were described in Section 2: choose $h_{1}=1, \ldots, h_{r} \in H\left(\mathbb{A}_{f}\right)$ such that

$$
H_{+}(\mathbb{Q}) \backslash H\left(\mathbb{A}_{f}\right) / K=\coprod_{j=1}^{r} H_{+}(\mathbb{Q}) h_{j} K .
$$

For $h \in H\left(\mathbb{A}_{f}\right)$, we write $\Gamma_{h}=H_{+}(\mathbb{Q}) \cap h K h^{-1}$ (and $\Gamma=\Gamma_{1}$ ). Then

$$
X_{K} \cong \coprod_{j=1}^{r} \Gamma_{h_{j}} \backslash \mathbb{D}^{+} .
$$

Let $v, w \in V(F)$ span a totally positive definite plane $U$ and recall that we denote by $H_{U} \subset H$ the pointwise stabilizer of $U$. For $h \in H\left(\mathbb{A}_{f}\right)$, let $K_{U, h}=H_{U}\left(\mathbb{A}_{f}\right) \cap h K h^{-1}$. Choose coset representatives $h_{i}^{\prime} \in H_{U}\left(\mathbb{A}_{f}\right)$ such that

$$
\left(H_{U}\right)_{+}(\mathbb{Q}) \backslash H_{U}\left(\mathbb{A}_{f}\right) / K_{U, h}=\coprod_{i=1}^{s}\left(H_{U}\right)_{+}(\mathbb{Q}) h_{i}^{\prime} K_{U, h},
$$

and write $h_{i}^{\prime} h=\gamma_{i} h_{j} k_{i}$ with $\gamma_{i} \in H_{+}(\mathbb{Q}), k_{i} \in K$ and $h_{j}=h_{j(i)}$ a coset representative 
as in (2-8). Note that the double coset $\left(H_{U}\right)_{+}(\mathbb{Q}) \gamma_{i} \Gamma_{h_{j}}$ is well defined, that is, it is independent of the choice of $h_{i}^{\prime}$ and decomposition $h_{i}^{\prime} h=\gamma_{i} h_{j} k_{i}$.

Definition 3.13. Assume that $n>2$. We define $\Phi(v, w, h, s)_{K}$ to be the section of $\bigwedge^{2} T^{*}\left(X_{K}\right)$ whose restriction to the connected component $\Gamma_{h_{j}} \backslash \mathbb{D}^{+}$is

$$
\sum_{i \rightarrow j} \Phi\left(\gamma_{i}^{-1} v, \gamma_{i}^{-1} w, h_{j}, s\right)_{\Gamma},
$$

where the sum runs over those $i$ such that $j(i)=j$.

Note that this is well defined because of the invariance property (3-26). For $n=2$ we give a different definition. Namely, assume that $n=2$ and choose $\gamma_{0}$ in $H(\mathbb{Q})$ such that $\gamma_{0}^{-1} \mathbb{D}_{U}^{+}=\mathbb{D}_{U}^{-}$. With $h_{i}^{\prime}$ as in (3-39), write $\gamma_{0} h_{i}^{\prime} h=\gamma_{i_{0}} h_{j_{0}} k_{i_{0}}$ with $\gamma_{i_{0}} \in H_{+}(\mathbb{Q}), k_{i_{0}} \in K$ and $h_{j_{0}}=h_{j_{0}\left(i_{0}\right)}$ a coset representative as in (2-8). As above, the double coset $\left(H_{U}\right)_{+}(\mathbb{Q}) \gamma_{i 0} \Gamma_{h_{j_{0}}}$ is well defined.

Definition 3.14. Assume that $n=2$. We define $\Phi(v, w, h, s)_{K}$ to be the section of $\wedge^{2} T^{*}\left(X_{K}\right)$ whose restriction to the connected component $\Gamma_{h_{j}} \backslash \mathbb{D}^{+}$is

$$
\sum_{i \rightarrow j} \Phi\left(\gamma_{i}^{-1} v, \gamma_{i}^{-1} w, h_{j}, s\right)_{\Gamma}+\sum_{i_{0} \rightarrow j} \Phi\left(\gamma_{i_{0}}^{-1} v, \gamma_{i_{0}}^{-1} w, h_{j}, s\right)_{\Gamma},
$$

where the sums run over those $i$ and $i_{0}$ such that $j(i)=j$ and $j_{0}\left(i_{0}\right)=j$, respectively.

The forms $\Phi(v, w, h, s)_{K}$ are locally integrable on $X_{K}$. We denote by

$$
\left[\Phi(v, w, h, s)_{K}\right] \in \mathscr{D}^{1,1}\left(X_{K}\right)
$$

the corresponding current on $X_{K}$.

We also define a current

$$
\left[\Phi(v, w, h)_{K}\right] \in \mathscr{D}^{1,1}\left(X_{K}\right)
$$

whose restriction to the connected component $\Gamma_{h_{j}} \backslash \mathbb{D}^{+}$is

$$
\begin{array}{rr}
\sum_{i \rightarrow j}\left[\Phi\left(\gamma_{i}^{-1} v, \gamma_{i}^{-1} w, h_{j}\right)_{\Gamma}\right] & \text { if } n>2, \\
\sum_{i \rightarrow j}\left[\Phi\left(\gamma_{i}^{-1} v, \gamma_{i}^{-1} w, h_{j}\right)_{\Gamma}\right]+\sum_{i_{0} \rightarrow j}\left[\Phi\left(\gamma_{i_{0}}^{-1} v, \gamma_{i_{0}}^{-1} w, h_{j}\right)_{\Gamma}\right] & \text { if } n=2,
\end{array}
$$

with the currents in the sum as in (3-32). See Section 4B3 for an example.

Remark 3.15. The above definitions reflect the structure of the connected components of the special cycles $Z(v, w, h)_{K}$ in Section 2B. Namely, let $v, w \in V(F)$ be vectors spanning a totally positive definite plane and $h \in H\left(\mathbb{A}_{f}\right)$. Attached to such 
a pair there are Shimura varieties $X(v, w, h)_{K}$ and $X(v, h)_{K}$ (see (2-13)) together with proper maps

$$
X(v, w, h)_{K} \stackrel{\iota}{\longrightarrow} X(v, h)_{K} \stackrel{f}{\longrightarrow} X_{K} .
$$

Then $\iota_{*}\left(\left[X(v, w, h)_{K}\right]\right)$ defines a divisor on $X(v, h)_{K}$, and a finite sum of functions of the form (3-9) defines a Green function $G(v, w, h)_{K}$ on $X(v, h)_{K}$ with a logarithmic singularity along $\iota_{*}\left(\left[X(v, w, h)_{K}\right]\right)$. Writing $\left[G(v, w, h)_{K}\right]$ for the current in $\mathscr{D}^{0}\left(X_{K}\right)$ associated with $G(v, w, h)_{K}$, it follows from Kudla's description of the connected components of the cycles $Z(v, w, h)_{K}$ (see [Kudla 1997, Lemma 4.1]) that

$$
\left[\Phi(v, w, h)_{K}\right]=2 \pi i \cdot f_{*}\left(\left[G(v, w, h)_{K}\right]\right) .
$$

Some basic properties of the forms $\Phi(v, w, h, s)_{K}$ are summarized in the next lemma; these properties are analogous to those of special cycles proved in [Kudla 1997, Lemma 2.2]. Recall that for every $h \in H\left(\mathbb{A}_{f}\right)$ there is a map

$$
r(h): X_{h K h^{-1}} \longrightarrow X_{K}
$$

sending $H(\mathbb{Q})\left(z, h^{\prime}\right) h K h^{-1}$ to $H(\mathbb{Q})\left(z, h^{\prime} h\right) K$. The map $r(h)$ is an isomorphism of complex manifolds, and we denote by $\Phi \mapsto \Phi \cdot h$ the induced map defined on sections of the bundle of differential forms.

Lemma 3.16. (1) $\Phi(v, w, h k, s)_{K}=\Phi(v, w, h, s)_{K}$ for all $k \in K$.

(2) $\Phi\left(v, w, h_{U} h, s\right)_{K}=\Phi(v, w, h, s)_{K}$ for all $h_{U} \in H_{U}\left(\mathbb{A}_{f}\right)$.

(3) $\Phi(\gamma v, \gamma w, \gamma h, s)_{K}=\Phi(v, w, h, s)_{K}$ for all $\gamma \in H(\mathbb{Q})$.

(4) $\Phi\left(v, w, h_{1} h^{-1}, s\right)_{h K h^{-1}} \cdot h=\Phi\left(v, w, h_{1}, s\right)_{K}$ for all $h_{1}, h \in H\left(\mathbb{A}_{f}\right)$.

Proof. Part (1) is obvious. Part (2) follows from the fact that for any complete set $\left\{h_{i}^{\prime} \mid i=1, \ldots, s\right\}$ of coset representatives for

$$
S(U, h, K)=\left(H_{U}\right)_{+}(\mathbb{Q}) \backslash H_{U}\left(\mathbb{A}_{f}\right) / K_{U, h},
$$

the set $\left\{h_{i}^{\prime} h_{U}^{-1} \mid i=1, \ldots, s\right\}$ is a complete set of representatives for $S\left(U, h_{U} h, K\right)$. To prove part (3), note that given any set $\left\{h_{i}^{\prime} \mid i=1, \ldots, s\right\}$ as above and any $\gamma \in H(\mathbb{Q})$, the elements $\gamma h_{i}^{\prime} \gamma^{-1}$ for $i=1, \ldots, s$ form a complete set of representatives for $S(\gamma(U), \gamma h, K)$, so that writing $\gamma h_{i}^{\prime} \gamma^{-1} \cdot(\gamma h)=\left(\gamma \gamma_{i}\right) h_{j} k_{i}$ with $j=j(i)$ leads to

$$
\begin{aligned}
\left.\Phi(\gamma v, \gamma w, \gamma h, s)_{K}\right|_{\Gamma_{h_{j}} \backslash \mathbb{D}^{+}} & =\sum_{i \rightarrow j} \Phi\left(\left(\gamma \gamma_{i}\right)^{-1} \gamma v,\left(\gamma \gamma_{i}\right)^{-1} \gamma w, z, s\right)_{\Gamma_{h_{j}}} \\
& =\sum_{i \rightarrow j} \Phi\left(\gamma_{i}^{-1} v, \gamma_{i}^{-1} w, z, s\right)_{\Gamma_{h_{j}}}=\left.\Phi(v, w, h, s)_{K}\right|_{\Gamma_{h_{j} \backslash \mathbb{D}^{+}}},
\end{aligned}
$$

as was to be shown. Finally, (4) follows from the fact that if $\left\{h_{j} \mid j=1, \ldots, r\right\}$ is 
a set of coset representatives for $H_{+}(\mathbb{Q}) \backslash H\left(\mathbb{A}_{f}\right) / K$, then $\left\{h_{j} h^{-1} \mid j=1, \ldots, r\right\}$ is a set of coset representatives for $H_{+}(\mathbb{Q}) \backslash H\left(\mathbb{A}_{f}\right) / h K h^{-1}$.

Assume that $K^{\prime} \subset K$, with $K^{\prime}$ an open compact subgroup of $H\left(\mathbb{A}_{f}\right)$ and let pr: $X_{K^{\prime}} \rightarrow X_{K}$ be the natural projection map. The following lemma computes $\operatorname{pr}^{*}\left(\Phi(v, w, h, s)_{K}\right)$.

Lemma 3.17. Let $K^{\prime} \subset K$ be as above. Then

$$
\operatorname{pr}^{*}\left(\Phi(v, w, h, s)_{K}\right)=\sum_{k \in h^{-1} K_{U, h} h \backslash K / K^{\prime}} \Phi(v, w, h k, s)_{K^{\prime}} .
$$

Proof. Note that the sum on the right hand side is well defined by (1) and (2) of Lemma 3.16. Now consider the restriction of $\Phi(v, w, h, s)_{K}$ to $\Gamma_{h_{j}} \backslash \mathbb{D}^{+}$. By definition, this is the sum

$$
\sum_{i \in I} \Phi\left(\gamma_{i}^{-1} v, \gamma_{i}^{-1} w, h, s\right)_{\Gamma_{h_{j}}},
$$

where $\gamma_{i} \in H_{+}(\mathbb{Q})$ satisfies $\gamma_{i} h_{j} k_{i}=h_{i}^{\prime} h$ for some $k_{i} \in K$ and $h_{i}^{\prime} \in H_{U}\left(\mathbb{A}_{f}\right)$, with $\left\{h_{i}^{\prime} \mid i \in I\right\}$ a complete set of representatives of the double coset

$$
\left(H_{U}\right)_{+}(\mathbb{Q}) \backslash H_{U}\left(\mathbb{A}_{f}\right) \cap H_{+}(\mathbb{Q}) h_{j} K h^{-1} / K_{U, h} .
$$

Assume first that $n>2$. By [Kudla 1997, Lemma 5.7(i)], this double coset is in bijection with the set of $\Gamma_{h_{j}}$-orbits in

$$
S\left(v, w, h_{j} K h^{-1}\right):=H_{+}(\mathbb{Q}) \cdot(v, w) \cap h_{j} K h^{-1} \cdot(v, w) .
$$

The bijection sends $\Gamma_{h_{j}} \cdot\left(v_{i}, w_{i}\right)$, where $\left(v_{i}, w_{i}\right)=\gamma_{i} \cdot(v, w)=h_{j} k_{i} h^{-1} \cdot(v, w)$ with $\gamma_{i} \in H_{+}(\mathbb{Q})$ and $k \in K$, to the double coset $\left(H_{U}\right)_{+}(\mathbb{Q}) \gamma_{i}^{-1} h_{j} k_{i} h^{-1} K_{U, h}$. Substituting the definition of $\Phi(v, w, h, s)_{\Gamma_{h_{j}}}$, we see that the restriction of $\frac{1}{2} \Phi(v, w, h, s)_{K}$ to $\Gamma_{h_{j}} \backslash \mathbb{D}^{+}$is given by

$$
\sum_{\left(v^{\prime}, w^{\prime}\right) \in S\left(v, w, h_{j} K h^{-1}\right)} \omega\left(v^{\prime}, w^{\prime}, z, s\right) .
$$

This sum can be rewritten as

$$
\sum_{k \in h^{-1} K_{U, h} h \backslash K / K^{\prime}} \sum_{\left(v^{\prime}, w^{\prime}\right) \in S\left(v, w, h_{j} K^{\prime}(h k)^{-1}\right)} \omega\left(v^{\prime}, w^{\prime}, z, s\right)
$$

and the claim follows directly from this. The proof for $n=2$ proceeds similarly by using [Kudla 1997, Lemma 5.7(ii)].

Analogous statements to those in Lemma 3.16 and Lemma 3.17 hold for the currents $\left[\Phi(v, w, h, s)_{K}\right]$ and $\left[\Phi(v, w, h)_{K}\right]$. 
3G. Weighted currents. Following Kudla's [1997] definition of weighted cycles we introduce currents in $\mathscr{D}^{1,1}(X)=\lim _{\longleftarrow} \mathscr{D}^{1,1}\left(X_{K}\right)$ as finite sums of the currents $\left[\Phi(v, w, h, s)_{K}\right]$ above weighted by the values of a Schwartz function $\mathscr{S}\left(V\left(\mathbb{A}_{f}\right)^{2}\right)$.

Given a totally positive definite symmetric matrix $T \in \operatorname{Sym}_{2}(F)$, let

$$
\Omega_{T}\left(\mathbb{A}_{f}\right)=\left\{(v, w) \in V\left(\mathbb{A}_{f}\right)^{2} \mid T(v, w)=T\right\},
$$

where $T(v, w)$ is defined in (3-22). Assume that $\Omega_{T}\left(\mathbb{A}_{f}\right) \neq \varnothing$. Then there exists $\left(v_{0}, w_{0}\right) \in \Omega_{T}\left(\mathbb{A}_{f}\right) \cap V(F)^{2}$ by the Hasse principle for quadratic forms. Moreover, the action of $H\left(\mathbb{A}_{f}\right)$ on $\Omega_{T}\left(\mathbb{A}_{f}\right)$ is transitive by Witt's theorem. Let $K$ be a compact open subgroup of $H\left(\mathbb{A}_{f}\right)$. The orbits of $K$ on $\Omega_{T}\left(\mathbb{A}_{f}\right)$ are open, and so if $\varphi \in \mathscr{S}\left(V\left(\mathbb{A}_{f}\right)^{2}\right)$ is invariant under $K$, we have

$$
\operatorname{Supp}(\varphi) \cap \Omega_{T}\left(\mathbb{A}_{f}\right)=\coprod_{i=1}^{k} K \xi_{i}^{-1} \cdot\left(v_{0}, w_{0}\right)
$$

for some elements $\xi_{1}, \ldots, \xi_{k} \in H\left(\mathbb{A}_{f}\right)$.

Definition 3.18. Let $T \in \operatorname{Sym}_{2}(F)$ be a totally positive definite matrix and let $\varphi \in \mathscr{S}\left(V\left(\mathbb{A}_{f}\right)^{2}\right)$ be fixed by $K$. With $\left(v_{0}, w_{0}\right)$ and $\xi_{i}$ as above and $\operatorname{Re}(s) \gg 0$, define

$$
\Phi(T, \varphi, s)_{K}=\sum_{i=1}^{k} \varphi\left(\xi_{i}^{-1} \cdot\left(v_{0}, w_{0}\right)\right) \cdot \Phi\left(v_{0}, w_{0}, \xi_{i}, s\right)_{K} .
$$

We denote by $\left[\Phi(T, \varphi, s)_{K}\right]$ the corresponding current in $\mathscr{D}^{1,1}\left(X_{K}\right)$.

Note that $\Phi(T, \varphi, s)_{K}$ is independent of the choice of $\left\{\xi_{1}, \ldots, \xi_{k}\right\}$ by (1) and (2) of Lemma 3.16. The behavior of $\Phi(T, \varphi, s)_{K}$ under pullbacks coming from compact subgroups $K^{\prime} \subset K$ is simpler than that of the forms $\Phi(v, w, h, s)_{K}$ defined above. The next proposition proves this and an equivariance property for the action of $H\left(\mathbb{A}_{f}\right)$.

Proposition 3.19. (1) Let $K^{\prime} \subset K$ be an open compact subgroup of $H\left(\mathbb{A}_{f}\right)$ and consider the natural map $\mathrm{pr}: X_{K^{\prime}} \rightarrow X_{K}$. Then

$$
\operatorname{pr}^{*}\left(\Phi(T, \varphi, s)_{K}\right)=\Phi(T, \varphi, s)_{K^{\prime}} .
$$

(2) For any $h \in H\left(\mathbb{A}_{f}\right)$, we have

$$
\Phi(T, \omega(h) \varphi, s)_{h K h^{-1}}=\Phi(T, \varphi, s)_{K} \cdot h^{-1},
$$

where $\omega(h) \varphi$ is the Schwartz function given by $\omega(h) \varphi(v, w)=\varphi\left(h^{-1} v, h^{-1} w\right)$.

Proof. To prove (1), let $\left(v_{0}, w_{0}\right) \in \Omega_{T}(F)$ and denote by $H_{U}$ the pointwise stabilizer in $H$ of the plane spanned by $v_{0}$ and $w_{0}$. Note that the map $h \mapsto h^{-1} \cdot\left(v_{0}, w_{0}\right)$ 
induces a bijection $H_{U}\left(\mathbb{A}_{f}\right) \backslash H\left(\mathbb{A}_{f}\right) \cong \Omega_{T}\left(\mathbb{A}_{f}\right)$. Now we use Lemma 3.17 and obtain

$$
\begin{aligned}
\operatorname{pr}^{*}\left(\Phi(T, \varphi, s)_{K}\right) & \sum_{h \in H_{U}\left(\mathbb{A}_{f}\right) \backslash H\left(\mathbb{A}_{f}\right) / K} \omega(h) \varphi\left(v_{0}, w_{0}\right) \operatorname{pr}^{*}\left(\Phi\left(v_{0}, w_{0}, h, s\right)_{K}\right) \\
= & \sum_{h \in H_{U}\left(\mathbb{A}_{f}\right) \backslash H\left(\mathbb{A}_{f}\right) / K} \sum_{k \in h^{-1} K_{U, h} h \backslash K / K^{\prime}} \omega(h) \varphi\left(v_{0}, w_{0}\right) \Phi(v, w, h k, s)_{K^{\prime}} \\
& =\sum_{h \in H_{U}\left(\mathbb{A}_{f}\right) \backslash H\left(\mathbb{A}_{f}\right) / K^{\prime}} \omega(h) \varphi\left(v_{0}, w_{0}\right) \Phi\left(v_{0}, w_{0}, h, s\right)_{K^{\prime}}=\Phi(T, \varphi, s)_{K^{\prime}} .
\end{aligned}
$$

Part (2) follows directly from part (4) of Lemma 3.16.

We can also define a weighted version of the currents $\left[\Phi(v, w, h)_{K}\right]$ in (3-43). Namely, for $T \in \operatorname{Sym}_{2}(F)_{\gg 0}$ and $\varphi \in \mathscr{S}\left(V\left(\mathbb{A}_{f}\right)^{2}\right)$ fixed by $K$ as above and $\xi_{i}$ as in (3-48), let

$$
\left[\Phi(T, \varphi)_{K}\right]=\sum_{i=1}^{k} \varphi\left(\xi_{i}^{-1} \cdot\left(v_{0}, w_{0}\right)\right) \cdot\left[\Phi\left(v_{0}, w_{0}, \xi_{i}\right)_{K}\right] \in \mathscr{D}^{1,1}\left(X_{K}\right) .
$$

See Section 4B4 for an example. It follows from (1) in Proposition 3.19 that the currents $\left[\Phi(T, \varphi, s)_{K}\right]$ and $\left[\Phi(T, \varphi)_{K}\right]$ are compatible under inclusions $K^{\prime} \subset K$ and hence one can define

$$
\begin{aligned}
{[\Phi(T, \varphi, s)] } & =\left(\left[\Phi(T, \varphi, s)_{K}\right]\right)_{K} \in \mathscr{D}^{1,1}(X)={\underset{K}{K}}_{\lim } \mathscr{D}^{1,1}\left(X_{K}\right), \\
{[\Phi(T, \varphi)] } & =\left(\left[\Phi(T, \varphi)_{K}\right]\right)_{K} \in \mathscr{D}^{1,1}(X) .
\end{aligned}
$$

Moreover, the space $\mathscr{D}^{1,1}(X)$ carries a natural left action of $H\left(\mathbb{A}_{f}\right)$ induced by the maps $r(h)^{-1}: X_{K} \rightarrow X_{h K h^{-1}}$; we denote the action of $h \in H\left(\mathbb{A}_{f}\right)$ on $\Phi \in \mathscr{D}^{1,1}(X)$ by $\Phi \cdot r(h)^{-1}$. Then, for any $h \in H\left(\mathbb{A}_{f}\right)$, we have

$$
\begin{aligned}
{[\Phi(T, \omega(h) \varphi, s)] } & =[\Phi(T, \varphi, s)] \cdot r(h)^{-1}, \\
{[\Phi(T, \omega(h) \varphi)] } & =[\Phi(T, \varphi)] \cdot r(h)^{-1} .
\end{aligned}
$$

That is, the assignments $T \otimes \varphi \mapsto[\Phi(T, \varphi, s)]$ and $T \otimes \varphi \mapsto[\Phi(T, \varphi)]$ induce $H\left(\mathbb{A}_{f}\right)$-equivariant linear maps

$$
\mathbb{C}\left[\operatorname{Sym}_{2}(F)_{>0}\right] \otimes \mathscr{S}\left(V\left(\mathbb{A}_{f}\right)^{2}\right) \longrightarrow \mathscr{D}^{1,1}(X) .
$$

3H. A regularized theta lift. From now on and to avoid dealing with metaplectic groups, we will assume that $V$ has even dimension over $F$. Our next goal is to show that, for $\operatorname{Re}(s) \gg 0$, the form $\Phi(T, \varphi, s)$ can be obtained as a regularized theta 
lift. More precisely, below we introduce a function $\mathscr{M}_{T}(g, s)$ defined on a certain subgroup of $\operatorname{Sp}_{4}\left(\mathbb{A}_{F}\right)$ and a theta function $\theta(g ; \varphi)$ that takes values in $\mathscr{A}^{1,1}(X)$. We then define a regularized theta lift $\left(\mathscr{M}_{T}(s), \theta(\cdot ; \varphi)\right)^{\text {reg }}$. The main result of this section (Proposition 3.21) shows that the regularized theta lift converges on an open dense subset of $X$ and moreover agrees with $\Phi(T, \varphi, s)$ there. The next two subsections define the functions just mentioned.

3H1. Schwartz forms. For $z \in \mathbb{D}$, note that the map $v \mapsto Q\left(v_{z^{\perp}}\right)-Q\left(v_{z}\right)$ defines a positive definite quadratic form on $V_{1}$. We write

$$
\varphi^{0}(v, z)=e^{-2 \pi\left(Q\left(v_{z} \perp\right)-Q\left(v_{z}\right)\right)}
$$

for the Gaussian associated with $z$. Note that $\varphi^{0}(v, z)$ lies in $\mathscr{S}\left(V_{1}\right) \otimes \mathscr{C}^{\infty}(\mathbb{D})$ and that it is fixed by $H(\mathbb{R})$, i.e., $\varphi^{0}(h x, h z)=\varphi^{0}(x, z)$ for every $h \in H(\mathbb{R})$. Now define

$$
\varphi^{1,1}(v, w, z) \in\left[\mathscr{S}\left(V_{1}^{2}\right) \otimes \mathscr{A}^{1,1}(\mathbb{D})\right]^{H(\mathbb{R})}
$$

by

$$
\begin{aligned}
\varphi^{1,1}(v, w, z) & =\bar{\partial}\left(\varphi^{0}(w, z) \partial \varphi^{0}(v, z)\right) \\
& =\bar{\partial} \varphi^{0}(w, z) \wedge \partial \varphi^{0}(v, z)+\varphi^{0}(w, z) \bar{\partial} \partial \varphi^{0}(v, z) .
\end{aligned}
$$

For a quadratic vector space $(W, Q)$ with positive definite quadratic form, let $\varphi_{+}^{0}(v, w) \in \mathscr{S}\left(W^{2}\right)$ be the standard Gaussian defined by

$$
\varphi_{+}^{0}(v, w)=e^{-2 \pi(Q(v)+Q(w))} .
$$

For $v \in V(\mathbb{R})$, denote by $v_{i}, i=1, \ldots, d$ the image of $v$ under the natural map $V(\mathbb{R}) \rightarrow V \otimes_{F, \sigma_{i}} \mathbb{R}$. Define

$$
\varphi_{\infty}^{1,1} \in\left[\mathscr{S}\left(V(\mathbb{R})^{2}\right) \otimes \mathscr{A}^{1,1}(\mathbb{D})\right]^{H(\mathbb{R})}
$$

by

$$
\varphi_{\infty}^{1,1}(v, w, z)=\varphi^{1,1}\left(v_{1}, w_{1}, z\right) \otimes \varphi_{+}^{0}\left(v_{2}, w_{2}\right) \otimes \cdots \otimes \varphi_{+}^{0}\left(v_{d}, w_{d}\right) .
$$

Denote by $\omega=\omega_{\psi}$ the Weil representation of $\operatorname{Sp}_{4}\left(\mathbb{A}_{F}\right)$ on $\mathscr{S}\left(V(\mathbb{A})^{2}\right)$ with respect to our fixed character $\psi$ (see, e.g., [Kudla and Rallis 1988] for explicit formulas). For $g=\left(g_{f}, g_{\infty}\right) \in \operatorname{Sp}_{4}\left(\mathbb{A}_{F}\right), h \in H\left(\mathbb{A}_{f}\right)$ and $\varphi \in \mathscr{S}\left(V\left(\mathbb{A}_{f}\right)^{2}\right)$ fixed by an open compact subgroup $K$ of $H\left(\mathbb{A}_{f}\right)$, the theta function

$$
\theta(g ; \varphi)_{K}=\sum_{(v, w) \in V(F)^{2}} \omega\left(g_{f}\right) \varphi(v, w) \cdot \omega\left(g_{\infty}\right) \varphi_{\infty}^{1,1}(v, w)
$$

defines a $(1,1)$-form on $X_{K}$. 
3H2. Regularized lifts. Let $\kappa=\frac{n+2}{2}$. For $a \in \mathbb{R}_{>0}$, define

$$
W_{a}(y)=\frac{(4 \pi a)^{\kappa-1}}{\Gamma(\kappa-1)} \cdot y^{\kappa / 2} e^{-2 \pi a y}, \quad y>0 .
$$

Note that

$$
\int_{0}^{\infty} W_{a}(y) y^{\kappa / 2} e^{-2 \pi a y} \frac{d y}{y^{2}}=1 .
$$

Consider the following subgroups of $\mathrm{Sp}_{4, F}$ :

$$
\begin{aligned}
& N(k)=\left\{n=n(X)=\left(\begin{array}{cc}
1_{2} & X \\
& 1_{2}
\end{array}\right) \mid X={ }^{t} X \in \operatorname{Sym}_{2}(k)\right\}, \\
& A(k)=\left\{a=m(t, v)=\left(\begin{array}{llll}
y & & & \\
& t & & \\
& & y^{-1} & \\
& & & t^{-1}
\end{array}\right) \mid y, t \in k^{\times}\right\} .
\end{aligned}
$$

Let $d n$ be the unique Haar measure on $N(\mathbb{A})$ such that $\operatorname{Vol}(N(F) \backslash N(\mathbb{A}), d n)=1$. Denote by $A(\mathbb{R})^{0}$ the connected component of the identity in $A(\mathbb{R})$. Let $d a$ be the measure on $A(\mathbb{R})^{0}$ defined by

$$
\begin{aligned}
& \int_{A(\mathbb{R})^{0}} f(a) d a \\
& =\int_{\left(\mathbb{R}_{>0}\right)^{d}} \int_{\left(\mathbb{R}_{>0}\right)^{d}} f\left(m\left(y_{1}^{1 / 2}, t_{1}^{1 / 2}\right), \ldots, m\left(y_{d}^{1 / 2}, t_{d}^{1 / 2}\right)\right) \frac{d y_{1}}{y_{1}^{2}} \frac{d t_{1}}{t_{1}^{2}} \cdots \frac{d y_{d}}{y_{d}^{2}} \frac{d t_{d}}{t_{d}^{2}},
\end{aligned}
$$

where $d y_{i}, d t_{i}$ denote the Lebesgue measure.

For a matrix $T \in \operatorname{Sym}_{2}(F)$, define a character $\psi_{T}: N(F) \backslash N(\mathbb{A}) \rightarrow \mathbb{C}^{\times}$by $\psi_{T}(n(X))=\psi(\operatorname{tr}(T X))$. For such a symmetric matrix $T=\left(\begin{array}{ll}a & b \\ b & c\end{array}\right)$ and $i=1, \ldots, d$, we write

$$
\sigma_{i}(T)=\left(\begin{array}{ll}
a_{i} & b_{i} \\
b_{i} & c_{i}
\end{array}\right)
$$

where $a_{i}=\sigma_{i}(a), b_{i}=\sigma_{i}(b)$ and $c_{i}=\sigma_{i}(c)$. We also write $T^{\iota}=\left(\begin{array}{ll}c & b \\ b & a\end{array}\right)$.

Definition 3.20. For $T=\left(\begin{array}{cc}a & b \\ b & c\end{array}\right) \in \operatorname{Sym}_{2}(F)$ totally positive definite, the function

$$
\mathscr{M}_{T}(n a, s): N(F) \backslash N(\mathbb{A}) \times A(\mathbb{R})^{0} \longrightarrow \mathbb{C}
$$

is defined by

$$
\begin{aligned}
\mathscr{M}_{T}\left(n m\left(y^{1 / 2}, t^{1 / 2}\right), s\right)=\left(2 \kappa_{\operatorname{dim}(V)}^{-1}\right) \overline{\psi_{T}(n)} & M_{\sigma_{1}(T)}\left(y_{1}, s\right) M_{\sigma_{1}(T)^{\iota}}\left(t_{1}, s\right) \\
& \times\left(y_{1} t_{1}\right)^{1-\frac{\kappa}{2}} \prod_{i=2}^{d} W_{a_{i}}\left(y_{i}\right) W_{c_{i}}\left(t_{i}\right),
\end{aligned}
$$

where $\kappa_{4}=2$ and $\kappa_{n}=1$ for $n>5$. 
Given a measurable function $f: \operatorname{Sp}_{4}\left(\mathbb{A}_{F}\right) \rightarrow \mathbb{C}$ that satisfies $f(n g)=f(g)$ for all $n \in N(F)$, define

$$
\left(\mathscr{M}_{T}(s), f\right)^{\mathrm{reg}}=\int_{A(\mathbb{R})^{0}} \int_{N(F) \backslash N(\mathbb{A})} \mathscr{M}_{T}(n a, s) f(n a) d n d a,
$$

provided that the integral converges.

Proposition 3.21. Let $T \in \operatorname{Sym}_{2}(F)$ be a positive definite symmetric matrix, $\varphi$ be a Schwartz form in $\mathscr{S}\left(V\left(\mathbb{A}_{f}\right)^{2}\right)$ fixed by an open compact subgroup $K \subset H\left(\mathbb{A}_{f}\right)$ and $\theta(g ; \varphi)_{K}$ be the theta function defined in (3-59). Then there is a dense open set $U \subseteq X_{K}$ with complement of measure zero such that for $\operatorname{Re}(s) \gg 0$, the regularized theta lift

$$
\left(\mathscr{M}_{T}(s), \theta(\cdot ; \varphi)_{K}\right)^{\mathrm{reg}}
$$

converges and equals $\Phi(T, \varphi, s)_{K}$ on $U$.

Proof. The sum defining $\theta(n a ; \varphi)_{K}$ and the inner integral in $\left(\mathscr{M}_{T}(s), \theta(\cdot, h ; \varphi)_{K}\right)^{\text {reg }}$ unfold to

$$
\int_{A(\mathbb{R})^{0}} \mathscr{M}_{T}(a, s) \sum_{(v, w) \in \Omega_{T}(F)} \varphi(v, w) \omega(a) \varphi_{\infty}^{1,1}(v, w, z) d a
$$

Let

$$
\tilde{U}=\mathbb{D}-\bigcup_{(v, w) \in \Omega_{T}(F) \cap \operatorname{Supp}(\varphi)}\left(\mathbb{D}_{v} \cup \mathbb{D}_{w}\right)
$$

so that $\tilde{U}$ is an open dense subset of $\mathbb{D}$ whose complement has measure zero. By Fubini's theorem and Lemma 3.22 below, the sum and the integral can be interchanged whenever $z \in \tilde{U}$; thus the above equals

$$
\sum_{(v, w) \in \Omega_{T}(F)} \varphi(v, w) \int_{A(\mathbb{R})^{0}} \mathscr{M}_{T}(a, s) \omega(a) \varphi_{\infty}^{1,1}(v, w, z) d a .
$$

The integral can be computed using equations (3-23) and (3-61). We obtain

$$
\int_{A(\mathbb{R})^{0}} \mathscr{M}_{T}(a, s) \omega(a) \varphi_{\infty}^{1,1}(v, w, z) d a=2 \omega(v, w, z, s) .
$$

Assume first that $n>2$. Then $H_{+}(\mathbb{Q})$ acts transitively on $\Omega_{T}(F)$, by [Kudla 1997, Lemma 5.5]; fixing $\left(v_{0}, w_{0}\right) \in \Omega_{T}(F)$ we see that for $z \in \tilde{U}$, the integral $\left(\mathscr{M}_{T}(s), \theta(\cdot ; \varphi)_{K}\right)^{\text {reg }}$ equals

$$
I\left(v_{0}, w_{0}, \varphi, s\right):=\sum_{(v, w) \in H_{+}(\mathbb{Q}) \cdot\left(v_{0}, w_{0}\right)} \varphi(v, w) \cdot \omega(v, w, z, s) .
$$


With $h_{j}, j=1, \ldots, r$ as in (2-8), we have

$$
\left.I\left(v_{0}, w_{0}, \varphi, s\right)\right|_{\Gamma_{h_{j}} \backslash \mathbb{D}^{+}}=\sum_{(v, w) \in H_{+}(\mathbb{Q}) \cdot\left(v_{0}, w_{0}\right)} \omega\left(h_{j}\right) \varphi(v, w) \cdot \omega(v, w, z, s) .
$$

Let $\xi_{i}, i=1, \ldots, k$ be as in (3-48) and define

$$
S_{j, i}\left(v_{0}, w_{0}\right)=H_{+}(\mathbb{Q}) \cdot\left(v_{0}, w_{0}\right) \cap h_{j} K \xi_{i}^{-1} \cdot\left(v_{0}, w_{0}\right) .
$$

Note that $\omega\left(h_{j}\right) \varphi(v, w)=\varphi\left(\xi_{i}^{-1} \cdot\left(v_{0}, w_{0}\right)\right)$ for every $(v, w) \in S_{j, i}\left(v_{0}, w_{0}\right)$ and

$$
H_{+}(\mathbb{Q}) \cdot\left(v_{0}, w_{0}\right) \cap \operatorname{Supp}\left(\omega\left(h_{j}\right) \varphi\right)=\coprod_{i=1}^{k} S_{j, i}\left(v_{0}, w_{0}\right) .
$$

Hence

$$
\left.I\left(v_{0}, w_{0}, \varphi, s\right)\right|_{\Gamma_{h_{j}} \backslash \mathbb{D}^{+}}=\sum_{i=1}^{k} \varphi\left(\xi_{i}^{-1} \cdot\left(v_{0}, w_{0}\right)\right) \sum_{(v, w) \in S_{j, i}\left(v_{0}, w_{0}\right)} \omega(v, w, z, s) .
$$

Note that the set $S_{j, i}\left(v_{0}, w_{0}\right)$ is stable under $\Gamma_{h_{j}}=H_{+}(\mathbb{Q}) \cap h_{j} K h_{j}^{-1}$, so that we can write

$$
\begin{aligned}
\sum_{(v, w) \in S_{j, i}\left(v_{0}, w_{0}\right)} \omega(v, w, z, s) & =\sum_{(v, w) \in \Gamma_{h_{j} \backslash S_{j, i}\left(v_{0}, w_{0}\right)}} \sum_{\gamma \in\left(\Gamma_{h_{j}}\right)_{v, w} \backslash \Gamma_{h_{j}}} \omega\left(\gamma^{-1} v, \gamma^{-1} w, z, s\right) \\
& =\sum_{(v, w) \in \Gamma_{h_{j} \backslash S_{j, i}\left(v_{0}, w_{0}\right)}} \Phi(v, w, z, s)_{\Gamma_{h_{j}} .}
\end{aligned}
$$

By [Kudla 1997, Lemma 5.7(i)], the set of orbits $\Gamma_{h_{j}} \backslash S_{j, i}\left(v_{0}, w_{0}\right)$ is in bijection with the double coset (where we write $H_{U}$ for $H_{v_{0}, w_{0}}$ )

$$
\left(H_{U}\right)_{+}(\mathbb{Q}) \backslash H_{U}\left(\mathbb{A}_{f}\right) \cap H_{+}(\mathbb{Q}) h_{j} K \xi_{i}^{-1} / K_{U, \xi_{i}}
$$

Moreover, the bijection is as follows: Suppose $(v, w) \in S_{j, i}\left(v_{0}, w_{0}\right)$ is of the form $\gamma \cdot\left(v_{0}, w_{0}\right)=h_{j} k \xi^{-1} \cdot\left(v_{0}, w_{0}\right)$. Then $\Gamma_{h_{j}} \cdot(v, w)$ corresponds to the double coset $\left(H_{U}\right)_{+}(\mathbb{Q}) \gamma^{-1} h_{j} k \xi^{-1} K_{U, \xi_{i}}$. Thus, by definition of $\Phi(v, w, h, s)_{K}$ we have

$$
\sum_{(v, w) \in \Gamma_{h_{j}} \backslash S_{j, i}\left(v_{0}, w_{0}\right)} \Phi(v, w, z, s)_{\Gamma}=\left.\Phi\left(v_{0}, w_{0}, \xi_{i}, s\right)_{K}\right|_{\Gamma_{h_{j}} \backslash \mathbb{D}^{+}},
$$

and hence

$$
\left.I\left(v_{0}, w_{0}, \varphi, s\right)\right|_{\Gamma_{h_{j} \backslash \mathbb{D}^{+}}}=\left.\sum_{i=1}^{k} \varphi\left(\xi_{i}^{-1} \cdot\left(v_{0}, w_{0}\right)\right) \cdot \Phi\left(v_{0}, w_{0}, \xi_{i}, s\right)_{K}\right|_{\Gamma_{h_{j}} \backslash \mathbb{D}^{+}}
$$

for every $j$, as was to be shown.

Now assume that $n=2$. By [Kudla 1997, Lemma 5.5], the group $H_{+}(\mathbb{Q})$ acts with two orbits on $\Omega_{T}(F)$, and we have $\Omega_{T}(F)=H_{+}(\mathbb{Q}) \cdot\left(v_{0}, w_{0}\right) \amalg H_{+}(\mathbb{Q}) \gamma_{0} \cdot\left(v_{0}, w_{0}\right)$ 
for any $\gamma_{0} \in H(\mathbb{Q})$ that fixes the plane $U_{0}$ spanned by $\left(v_{0}, w_{0}\right)$ but reverses its orientation given by the ordered basis $\left\{v_{0}, w_{0}\right\}$. Thus, for $z \in \tilde{U}$, the integral $\left(\mathscr{M}_{T}(s), \theta(\cdot ; \varphi)_{K}\right)^{\text {reg }}$ equals

$$
I\left(v_{0}, w_{0}, \varphi, s\right)+I\left(\gamma_{0} \cdot\left(v_{0}, w_{0}\right), \varphi, s\right) .
$$

Define

$$
S_{j, i}\left(v_{0}, w_{0}, \gamma_{0}\right)=H_{+}(\mathbb{Q}) \gamma_{0} \cdot\left(v_{0}, w_{0}\right) \cap h_{j} K \xi_{i}^{-1} \cdot\left(v_{0}, w_{0}\right) .
$$

Then $S_{j, i}\left(v_{0}, w_{0}, \gamma_{0}\right)$ is stable under $\Gamma_{h_{j}}$ and one shows as above that

$$
\begin{aligned}
\left.I\left(\gamma_{0} \cdot\left(v_{0}, w_{0}\right), \varphi, s\right)\right|_{\Gamma_{h_{j}} \backslash \mathbb{D}^{+}} & \\
& =\sum_{i=1}^{k} \varphi\left(\xi_{i}^{-1} \cdot\left(v_{0}, w_{0}\right)\right) \sum_{(v, w) \in \Gamma_{h_{j}} \backslash S_{j, i}\left(v_{0}, w_{0}, \gamma_{0}\right)} \Phi(v, w, z, s)_{\Gamma_{h_{j}}} .
\end{aligned}
$$

Note that we can choose $\gamma_{0}$ so that $\gamma_{0} \cdot v_{0}=v_{0}$ and $\gamma_{0} \cdot w_{0}=-w_{0}$. Since $\omega(v, w, z, s)=\omega(v,-w, z, s)$, we conclude from [Kudla 1997, Lemma 5.7(ii)] that

$$
\begin{gathered}
\left.\Phi\left(v_{0}, w_{0}, \xi_{i}, s\right)_{K}\right|_{\Gamma_{h_{j}} \backslash \mathbb{D}^{+}} \\
=\sum_{(v, w) \in \Gamma_{h_{j} \backslash S_{j, i}}\left(v_{0}, w_{0}\right)} \Phi(v, w, z, s)_{\Gamma_{h_{j}}}+\sum_{(v, w) \in \Gamma_{h_{j}} \backslash S_{j, i}\left(v_{0}, w_{0}, \gamma_{0}\right)} \Phi(v, w, z, s)_{\Gamma_{h_{j}}},
\end{gathered}
$$

and the claim follows from this.

The next lemma completes the proof of Proposition 3.21.

Lemma 3.22. Let $T \in \operatorname{Sym}_{2}(F)$ be totally positive definite, $\varphi$ be a Schwartz form in $\mathscr{S}\left(V\left(\mathbb{A}_{f}\right)^{2}\right)$ and let

$$
\tilde{U}=\mathbb{D}-\bigcup_{(v, w) \in \Omega_{T}(F) \cap \operatorname{Supp}(\varphi)}\left(\mathbb{D}_{v} \cup \mathbb{D}_{w}\right) .
$$

Then, for $\operatorname{Re}(s) \gg 0$, the sum

$$
\sum_{(v, w) \in \Omega_{T}(F)}|\varphi(v, w)| \int_{A(\mathbb{R})^{0}}\left|\mathscr{M}_{T}(a, s)\right| \cdot\left\|\omega(a) \varphi_{\infty}^{1,1}(v, w, z)\right\| d a
$$

converges for every $z \in \tilde{U}$.

Proof. Let $(v, w) \in \Omega_{T}(F)$. It is enough to show that, for $\operatorname{Re}(s) \gg 0$ and any $\Gamma \subset H_{+}(\mathbb{R})$, the sum

$$
\sum_{\gamma \in \Gamma_{v, w} \backslash \Gamma} \int_{A(\mathbb{R})^{0}}\left|\mathscr{M}_{T}(a, s)\right| \cdot\left\|\omega(a) \varphi_{\infty}^{1,1}\left(\gamma^{-1} v, \gamma^{-1} w, z\right)\right\| d a
$$


converges for $z \in \mathbb{D}^{+}-\left(\Gamma \cdot \mathbb{D}_{v}^{+} \cup \Gamma \cdot \mathbb{D}_{w}^{+}\right)$, since (3-67) is a finite linear combination of sums of this form. Note that if $\omega(v, z)$ is any of the forms $\partial \varphi^{0}(v, z), \bar{\partial} \varphi^{0}(v, z)$ or $\partial \bar{\partial} \varphi^{0}(v, z)$, then we can write

$$
\|\omega(v, z)\|=\sum_{i}\left\|P_{i}(v, z)\right\| \cdot \varphi^{0}(v, z),
$$

where the sum over $i$ is finite and the functions $P_{i}(v, z)$ are polynomial functions of $v$ for fixed $z$ satisfying $\left\|P_{i}(h v, h z)\right\|=\left\|P_{i}(v, z)\right\|$ for every $h \in H(\mathbb{R})$. In particular, there exists a positive constant $C$ and a natural number $k$ (in fact, $k=2$ will do) such that $\left\|P_{i}(v, z)\right\| \leq C Q\left(v_{z^{\perp}}\right)^{k}$ for every $z \in \mathbb{D}^{+}$and every $v$ of fixed positive norm $Q(v)=m>0$. Now choose $\epsilon>0$ such that $\left|Q\left(\gamma^{-1} v\right)_{z}\right|>\epsilon$ and $\left|Q\left(\gamma^{-1} w\right)_{z}\right|>\epsilon$ for all $\gamma \in \Gamma$. Then there exists a constant $C_{\epsilon}>0$ such that

$$
\begin{aligned}
\int_{A(\mathbb{R})^{0}}\left|\mathscr{M}_{T}(a, s)\right| \cdot\left\|\omega(a) \varphi_{\infty}^{1,1}\left(\gamma^{-1} v, \gamma^{-1} w, z\right)\right\| d a & \\
& <C_{\epsilon}\left|Q\left(\gamma^{-1} v\right)_{z^{\perp}} \cdot Q\left(\gamma^{-1} w\right)_{z^{\perp}}\right|^{-\frac{s+s_{0}}{2}+k},
\end{aligned}
$$

and the claim follows as in the proof of Proposition 3.6.

Theorem 1.1 now follows from Proposition 3.12 and Proposition 3.21.

3I. Higher Chow groups and regulators. We next focus on the relationship between the currents $\Phi(T, \varphi)$ introduced above and the currents in the image of the regulator map

$$
r_{\mathscr{D}}: \mathrm{CH}^{2}\left(X_{K}, 1\right) \rightarrow \mathscr{D}^{1,1}\left(X_{K}\right) .
$$

Let us first recall the definitions of the higher Chow group $\mathrm{CH}^{2}\left(X_{K}, 1\right)$ and of the above map.

Let $Y$ be an irreducible algebraic variety defined over a field $k$. The group $\mathrm{CH}^{2}(Y, 1)$ is defined as a quotient

$$
\mathrm{CH}^{2}(Y, 1)=Z^{2}(Y, 1) / B^{2}(Y, 1) .
$$

An element $c \in Z^{2}(Y, 1)$ is a finite linear combination

$$
c=\sum_{i} a_{i} \cdot\left(\pi_{i}: Z_{i} \rightarrow Y, f_{i}\right),
$$

where $Z_{i}$ is a normal variety over $k$ of dimension $\operatorname{dim}(Y)-1, \pi_{i}$ is a generically finite proper map, $f_{i}$ is a meromorphic function on $Z_{i}$, and $a_{i} \in \mathbb{Q}$; it is also required that

$$
\sum_{i} a_{i} \cdot\left(\pi_{i}\right)_{*}\left(\operatorname{div} f_{i}\right)=0
$$

as a cycle of codimension 2 in $Y$. For a description of $B^{2}(Y, 1)$, see [Voisin 2002]. 
Suppose that $k \subseteq \mathbb{C}$. Define a map

$$
\begin{aligned}
r_{\mathscr{D}}: \mathrm{CH}^{2}(Y, 1) & \longrightarrow \mathscr{D}^{1,1}\left(Y_{\mathbb{C}}\right) \\
\sum_{i} a_{i} \cdot\left(\pi_{i}: Z_{i} \rightarrow Y, f_{i}\right) & \longmapsto 2 \pi i \sum a_{i} \cdot\left(\pi_{i}\right)_{*}\left(\left[\log \left|f_{i}\right|\right]\right),
\end{aligned}
$$

where $\left(\pi_{i}\right)_{*}\left(\log \left|f_{i}\right|\right) \in \mathscr{D}^{1,1}\left(Y_{\mathbb{C}}\right)$ is the current defined by

$$
\left(\left(\pi_{i}\right)_{*}\left(\log \left|f_{i}\right|\right), \alpha\right)=\int_{Z_{i}} \pi_{i}^{*}(\alpha) \cdot \log \left|f_{i}\right|
$$

for $\alpha \in \mathscr{A}_{C}^{2 \operatorname{dim}(Y)-2}\left(Y_{\mathbb{C}}\right)$. The map $r_{\mathscr{D}}$ is known as a regulator map; it is linear and its image defines a rational vector subspace of $\mathscr{D}^{1,1}\left(Y_{\mathbb{C}}\right)$. Note also that for any $c \in \mathrm{CH}^{2}(Y, 1)$, the current $r_{\mathscr{D}}(c)$ is $d d^{c}$-closed: this follows from the identity of currents

$$
d d^{c}\left(\pi_{i}\right)_{*}\left(\log \left|f_{i}\right|^{2}\right)=\delta_{\operatorname{div} f_{i}}
$$

and condition (3-71).

Note that the currents $[\Phi(T, \varphi)]$ in (3-50) are not $d d^{c}$-closed. In fact, for the currents $\left[\Phi(v, w)_{\Gamma}\right]$ in $(3-31)$, we have

$$
d d^{c}\left[\Phi(v, w)_{\Gamma}\right]=\delta_{Z(v, w)_{\Gamma}}+d d^{c} G(v, w)_{\Gamma} \cdot \delta_{X(v)_{\Gamma}} .
$$

Here $d d^{c} G(v, w)_{\Gamma}$ extends to a smooth 2-form defined on $X(v)_{\Gamma}$, and the current $d d^{c} G(v, w)_{\Gamma} \cdot \delta_{X(v)_{\Gamma}} \in \mathscr{D}^{1,1}\left(X_{\Gamma}\right)$ is defined by

$$
\left(d d^{c} G(v, w)_{\Gamma} \cdot \delta_{X(v)_{\Gamma}}, \alpha\right)=\int_{X(v)_{\Gamma}} d d^{c} G(v, w)_{\Gamma} \wedge \alpha,
$$

for $\alpha \in \mathscr{A}_{c}^{n-1, n-1}\left(X_{\Gamma}\right)$.

Since $\Phi(T, \varphi)$ is not $d d^{c}$-closed, it is not in the image of the regulator map defined above. It is natural to ask for necessary and sufficient conditions for a finite linear combination $\sum_{T, \varphi} a(T, \varphi)[\Phi(T, \varphi)]$ with $a(T, \varphi) \in \mathbb{Q}$ to belong to the image of the regulator. The next proposition proves a weak result in this direction when $n \geq 4$. It turns out that in this case being $d d^{c}$-closed is also sufficient.

Proposition 3.23. Assume that $n \geq 4$. Let $\Phi_{K}=\sum_{T, \varphi} a(T, \varphi)\left[\Phi(T, \varphi)_{K}\right] \in$ $\mathscr{D}^{1,1}\left(X_{K}\right)$, where the sum is finite and $a(T, \varphi) \in \mathbb{Q}$. Then $d d^{c} \Phi_{K}=0$ if and only if $\Phi_{K}=r_{\mathscr{D}}(c)$ for some $c \in \mathrm{CH}^{2}\left(X_{K}, 1\right)$.

Proof. Above we showed that $r_{\mathscr{D}}(c)$ is $d d^{c}$-closed for any $c \in \mathrm{CH}^{2}\left(X_{K}, 1\right)$. Now let $\Phi_{K}=\sum_{T, \varphi} a(T, \varphi)\left[\Phi(T, \varphi)_{K}\right]$ as in the statement and assume that $d d^{c} \Phi_{K}=0$. We compute

$$
0=d d^{c} \Phi_{K}=\sum_{T, \varphi} a(T, \varphi) \cdot\left(\delta_{Z(T, \varphi)_{K}}+\Psi(T, \varphi)_{K}\right)
$$


where $\Psi(T, \varphi)_{K} \in \mathscr{D}^{1,1}\left(X_{K}\right)$ is a current whose support is a finite union of special divisors on $X_{K}$. More precisely, we have

$$
\Psi(T, \varphi)_{K}=\sum_{i} d d^{c} G_{i} \cdot \delta_{X\left(v_{i}, h_{i}\right)_{K}}
$$

where the sum is finite and $G_{i}$ is a finite linear combination of Green functions of the form (3-6) on $X\left(v_{i}, h_{i}\right)_{K}$ with logarithmic singularities on special divisors. Since the currents $\Psi(T, \varphi)_{K}$ and $\delta_{Z(T, \varphi)_{K}}$ are supported in different codimensions, it follows from (3-76) that

$$
\begin{aligned}
\sum_{T, \varphi} a(T, \varphi) \cdot \delta_{Z(T, \varphi)_{K}} & =0, \\
\sum_{T, \varphi} a(T, \varphi) \cdot \Psi(T, \varphi)_{K} & =0 .
\end{aligned}
$$

(To see this, pick a basis of open neighborhoods $\left(U_{j}\right)_{j \geq 1}$ of $\bigcup_{T, \varphi} Z(T, \varphi)_{K}$ and compactly supported smooth functions $\phi_{j}: U_{j} \rightarrow[0,1]$ such that $\phi_{j} \mid Z(T, \varphi)_{K} \equiv 1$. For $\alpha \in \mathscr{A}_{c}^{n-2, n-2}\left(X_{K}\right)$, evaluate (3-76) on the sequence $\left(\phi_{j} \alpha\right)_{j \geq 1}$ and apply dominated convergence on each $X\left(v_{i}, h_{i}\right)_{K}$.) Write

$$
\sum_{T, \varphi} a(T, \varphi)\left[\Phi(T, \varphi)_{K}\right]=\sum_{i} G_{i} \delta_{X}\left(v_{i}, h_{i}\right)_{K},
$$

where the sum over $i$ is finite and $G_{i}$ is a Green function on $X\left(v_{i}, h_{i}\right)_{K}$. Now Equation (3-78) implies that the summand corresponding to a connected special divisor $X(v)_{\Gamma}$ in this sum is of the form $G(v, \Gamma) \delta_{X(v)_{\Gamma}}$, where $G(v, \Gamma)$ is a Green function on $X(v)_{\Gamma}$ that satisfies $d d^{c} G(v, \Gamma)=0$. Since $n \geq 4$, we have $H^{1}\left(X(v)_{\Gamma}, \mathbb{C}\right)=0$ (see [Vogan and Zuckerman 1984, Theorem 8.1]) and it follows that $G(v, \Gamma)=a(v, \Gamma) \log \left|f_{v, \Gamma}\right|$ for some meromorphic function $f_{v, \Gamma} \in k\left(X(v)_{\Gamma}\right)^{\times}$ and some $a(v, \Gamma) \in \mathbb{Q}$. Thus, denoting by $\pi_{v, \Gamma}$ the map $X(v)_{\Gamma} \rightarrow X_{K}$, we find that $\Phi_{K}=\sum_{v, \Gamma} a(v, \Gamma) \cdot\left(\pi_{v, \Gamma}\right)_{*}\left(\log \left|f_{v, \Gamma}\right|\right)$, where the sum is finite. Consider now the formal sum $\sum a(v, \Gamma) \cdot\left(\pi_{v, \Gamma}, f_{v, \Gamma}\right)$. By Equation (3-77), we have $\sum_{v, \Gamma} a(v, \Gamma) \cdot\left(\pi_{v, \Gamma}\right)_{*}\left(\operatorname{div} f_{v, \Gamma}\right)=0$ and hence it defines an element $c \in \mathrm{CH}^{2}\left(X_{K}, 1\right)$ satisfying $r_{\mathscr{D}}(c)=\Phi_{K}$.

3J. Evaluating currents on differential forms. Let $\alpha \in \mathscr{A}_{c}^{n-1, n-1}\left(X_{K}\right)$ be a compactly supported form. Since Proposition 3.21 shows that the forms $\Phi(T, \varphi, s)_{K}$ are theta lifts, one can try to evaluate

$$
\left[\Phi(T, \varphi, s)_{K}\right](\alpha)=\int_{X_{K}} \Phi(T, \varphi, s)_{K} \wedge \alpha
$$

by interchanging the integrals. However, this interchange is not justified since the resulting integrals are not absolutely convergent. In this section, we will introduce 
certain currents $[\tilde{\Phi}(T, \varphi, s)]$ closely related to the $[\Phi(T, \varphi, s)]$. These currents will be meromorphic in $s \in \mathbb{C}$ (modulo $\operatorname{Im}(\partial)+\operatorname{Im}(\bar{\partial})$ as before) and we will show that their constant term at $s=s_{0}$ is a certain $\mathbb{Q}$-linear combination of the $[\Phi(T, \varphi)]$. Moreover, following ideas in [Bruinier and Funke 2004], we will give an expression of these currents as regularized theta lifts that allows us to evaluate them by interchanging the integrals (see Proposition 3.27).

For a pair of vectors $(v, w) \in V(F)^{2}$ spanning a totally positive definite plane, consider the $(1,1)$-form

$$
\tilde{\omega}(v, w, z, s)=\phi(v, w, z, s) \partial \bar{\partial} \phi(w, v, z, s)
$$

in $\mathscr{A}^{1,1}\left(\mathbb{D}-\left(\mathbb{D}_{v} \cup \mathbb{D}_{w}\right)\right)$. The form $\tilde{\omega}(v, w, z, s)$ is related to the form $\omega(v, w, z, s)$ as follows:

$$
\begin{aligned}
& \omega(v, w, z, s)+\overline{\omega(w, v, z, s)} \\
& =\bar{\partial} \phi(w, v, z, s) \wedge \partial \phi(v, w, z, s)+\phi(w, v, z, s) \bar{\partial} \partial \phi(v, w, z, s) \\
& +\partial \phi(v, w, z, s) \wedge \bar{\partial} \phi(w, v, z, s)+\phi(v, w, z, s) \partial \bar{\partial} \phi(w, v, z, s) \\
& =\tilde{\omega}(v, w, z, s)-\tilde{\omega}(w, v, z, s) .
\end{aligned}
$$

For $\Gamma \subset H_{+}(\mathbb{R})$, define a $(1,1)$-form on $X_{\Gamma}$ by

$$
\tilde{\Phi}(v, w, z, s)_{\Gamma}=\sum_{\gamma \in \Gamma_{v, w} \backslash \Gamma} \tilde{\omega}\left(\gamma^{-1} v, \gamma^{-1} w, z, s\right) .
$$

The proofs of Propositions 3.6 and 3.9 apply to this sum and show that it converges normally on $X_{\Gamma}-\left(X(v)_{\Gamma} \cup X(w)_{\Gamma}\right)$ and defines a locally integrable $(1,1)$ form on $X_{\Gamma}$. We define forms $\tilde{\Phi}(v, w, h, s)_{K}$ and $\tilde{\Phi}(T, \varphi, s)_{K}$ as in Section $3 \mathrm{~F}$ and Section 3G by replacing $\omega(v, w, z, s)$ with $\tilde{\omega}(v, w, z, s)$ throughout. As before, denote by $\left[\tilde{\Phi}(T, \varphi, s)_{K}\right]$ the current in $\mathscr{D}^{1,1}\left(X_{K}\right)$ corresponding to the form $\tilde{\Phi}(T, \varphi, s)_{K}$. The proof of Proposition 3.19 shows that the currents $\left[\tilde{\Phi}(T, \varphi, s)_{K}\right]$ for varying $K$ form a compatible system under the maps induced by inclusions $K^{\prime} \subset K$, so that we obtain a current $[\tilde{\Phi}(T, \varphi, s)] \in \mathscr{D}^{1,1}(X)=\varliminf_{K} \mathscr{D}^{1,1}\left(X_{K}\right)$.

Let us now describe the relation of the currents $[\tilde{\Phi}(T, \varphi, s)]$ with the currents $[\Phi(T, \varphi)]$. For $T=\left(\begin{array}{ll}a & b \\ b & c\end{array}\right) \in \operatorname{Sym}_{2}(F)_{>0}$ and $\varphi \in \mathscr{S}\left(V\left(\mathbb{A}_{f}\right)^{2}\right)$, define

$$
T^{\iota}=\left(\begin{array}{ll}
c & b \\
b & a
\end{array}\right), \quad \varphi^{\iota}(v, w)=\varphi(w, v) .
$$

Then it follows from Proposition 3.12 and Equation (3-80) that the image of the current $[\tilde{\Phi}(T, \varphi, s)]-\left[\tilde{\Phi}\left(T^{\iota}, \varphi^{\iota}, s\right)\right]$ in $\tilde{D}^{1,1}(X)$ admits meromorphic continuation to $s \in \mathbb{C}$ and that its constant term at $s=s_{0}=(n-1) / 2$ is given by

$$
\mathrm{CT}_{s=s_{0}}[\tilde{\Phi}(T, \varphi, s)]-\left[\tilde{\Phi}\left(T^{\iota}, \varphi^{\iota}, s\right)\right] \equiv[\Phi(T, \varphi)]-\left[\Phi\left(T^{\iota}, \varphi^{\iota}\right)\right],
$$


where $\equiv$ denotes equality of currents modulo $\partial+\bar{\partial}$. See Section 4B5 for an example of a current of this form.

Remark 3.24. From the point of view of regulator maps $r_{\mathscr{D}, K}: \mathrm{CH}^{2}\left(X_{K}, 1\right) \rightarrow$ $\mathscr{D}^{1,1}\left(X_{K}\right)$, the currents on the right hand side of Equation (3-83) are quite natural objects. Namely, let $[\Phi] \in \mathscr{D}^{1,1}\left(X_{\Gamma}\right)$ be any current of the form $[\Phi]=r_{\mathscr{D}}(c)$ with $c=\sum n_{i}\left(C_{i}, f_{i}\right) \in \mathrm{CH}^{2}\left(X_{\Gamma}, 1\right)$ (see Section 3I for definitions) such that the $C_{i}$ are special divisors and the $f_{i} \in k\left(C_{i}\right)^{\times} \otimes \mathbb{Q}$ are (pushforwards of) the meromorphic functions constructed by Bruinier [2012, Theorem 6.8]. Then condition (3-71) implies that $[\Phi]$ is a linear combination with $\mathbb{Q}$-coefficients of currents $\left[\Phi(v, w)_{\Gamma}\right]-$ $\left[\Phi(w, v)_{\Gamma}\right]$ for some pairs $(v, w) \in V(F)^{2}$. The current $[\Phi(T, \varphi)]-\left[\Phi\left(T^{\iota}, \varphi^{\iota}\right)\right]$ is just a finite sum of such currents, weighted by the values of $\varphi$.

Our next goal is to obtain an expression of $\tilde{\Phi}(T, \varphi, s)_{K}$ as a regularized theta lift with good convergence properties. To do so, we will use a relation between $\partial \bar{\partial} \varphi^{0}(v, z)$ and $\varphi_{K M}(v, z)$ established by Bruinier and Funke.

Denote by

$$
\varphi_{K M} \in\left[\mathscr{S}\left(V_{1}\right) \otimes \mathscr{A}^{1,1}(\mathbb{D})\right]^{H(\mathbb{R})}
$$

the $\mathscr{S}\left(V_{1}\right)$-valued, closed (1,1)-form constructed by Kudla and Millson [1986]. We have

$$
\varphi_{K M}(v, z)=P(v, z) \varphi^{0}(v, z)
$$

where $P(v, z) \in\left[\mathscr{C}^{\infty}\left(V_{1}\right) \otimes \mathscr{A}^{1,1}(\mathbb{D})\right]^{H(\mathbb{R})}$ is, for fixed $z$, a polynomial in $v$ of degree 2 (see [Kudla 1997, (7.16)] for an explicit description of $P(v, z)$; our $\varphi_{K M}$ is denoted $\varphi^{(1)}$ there).

Let $\tau=x+i y$ be an element of the upper half plane and let $g_{\tau}=\left(\begin{array}{cc}y^{1 / 2} & x y^{-1 / 2} \\ 0 & y^{-1 / 2}\end{array}\right) \in$ $\mathrm{SL}_{2}(\mathbb{R})$. Define

$$
\begin{gathered}
\varphi^{0}(v, \tau, z)=y^{-(n-2) / 4} \omega\left(g_{\tau}\right) \varphi^{0}(v, z)=y \cdot e\left(Q\left(v_{z^{\perp}}\right) \tau+Q\left(v_{z}\right) \bar{\tau}\right), \\
\varphi_{K M}(v, \tau, z)=y^{-(n+2) / 4} \omega\left(g_{\tau}\right) \varphi_{K M}(v, z) .
\end{gathered}
$$

Here $\omega$ denotes the Weil representation of $\mathrm{SL}_{2}(\mathbb{R})$ on $\mathscr{S}\left(V_{1}\right)$ and $e(x)=e^{2 \pi i x}$. Our presentation of $\left[\tilde{\Phi}(T, \varphi, s)_{K}\right]$ as a regularized theta lift will use the following result. Proposition 3.25 [Bruinier and Funke 2004, Theorem 4.4]. Let $L=-2 i \operatorname{Im}(\tau)^{2} \frac{\partial}{\partial \bar{\tau}}$ be the Maass lowering operator. Then

$$
d d^{c} \varphi^{0}(v, \tau, z)=-L \varphi_{K M}(v, \tau, z)
$$

where $d$ and $d^{c}=(4 \pi i)^{-1}(\partial-\bar{\partial})$ are the usual differential operators on $\mathbb{D}$.

Using this result, we can find a different expression for the form $\bar{\partial} \partial \phi(v, w, z, s)$. Let $L$ be the lowering operator in Proposition 3.25. For a symmetric positive definite 
matrix $T=\left(\begin{array}{ll}a & b \\ b & c\end{array}\right)$ and $\tau=x+i y \in \mathbb{U}$, define

$$
\tilde{M}_{T}(\tau, s)=4 \pi y^{2} \frac{\partial}{\partial \bar{\tau}}\left(M_{T}(y, s) e^{-2 \pi i a x}\right) .
$$

One computes

$$
\widetilde{M}_{T}(\tau, s)=\widetilde{C}(T, s) y^{1-k / 2} M_{1-k / 2, s / 2}\left(\left|\frac{4 \pi \operatorname{det} T}{c} y\right|\right) e^{\frac{2 \pi b^{2}}{c} y} e^{-2 \pi i a x},
$$

with $\widetilde{C}(T, s)=\pi i C(T, s) \cdot\left(s+s_{0}\right)$.

Lemma 3.26. For $v, w \in \Omega_{T}\left(V_{1}\right)$ and $\operatorname{Re}(s) \gg 0$, we have

$$
\bar{\partial} \partial \phi(v, w, z, s)=\int_{0}^{\infty} \tilde{M}_{T}(y, s) \varphi_{K M}(v, y, z) \frac{d y}{y^{2}} .
$$

Proof. Recall the integral expression for $\phi(v, w, z, s)$ given in (3-23). In terms of $\varphi^{0}(v, \tau, z)$, we have

$$
\begin{aligned}
\phi(v, w, z, s) & =\int_{0}^{\infty} M_{T}(y, s) \varphi^{0}(v, y, z) \frac{d y}{y^{2}} \\
& =\int_{0}^{\infty} \int_{0}^{1} M_{T}(y, s) e^{-2 \pi i Q(v) x} \varphi^{0}(v, \tau, z) \frac{d x d y}{y^{2}} .
\end{aligned}
$$

Using (3-88), we obtain

$$
\begin{aligned}
d d^{c} \phi(v, w, z, s) & =\int_{0}^{\infty} \int_{0}^{1} M_{T}(y, s) e^{-2 \pi i Q(v) x} d d^{c} \varphi^{0}(v, \tau, z) \frac{d x d y}{y^{2}} \\
& =-\int_{0}^{\infty} \int_{0}^{1} M_{T}(y, s) e^{-2 \pi i Q(v) x} L \varphi_{K M}(v, \tau, z) \frac{d x d y}{y^{2}} \\
& =-\int_{0}^{\infty} \int_{0}^{1} M_{T}(y, s) e^{-2 \pi i Q(v) x} \bar{\partial}\left(\varphi_{K M}(v, \tau, z) d \tau\right) \\
& =-\lim _{N \rightarrow \infty} \int_{\mathscr{F}_{N}} M_{T}(y, s) e^{-2 \pi i Q(v) x} \bar{\partial}\left(\varphi_{K M}(v, \tau, z) d \tau\right),
\end{aligned}
$$

where $\mathscr{F}_{N}=[0,1] \times\left[N^{-1}, N\right] \subset \mathbb{}$. Applying Stokes's Theorem, we find

$$
\begin{aligned}
d d^{c} \phi(v, w, z, s)= & \int_{0}^{\infty} \int_{0}^{1} \bar{\partial}\left(M_{T}(y, s) e^{-2 \pi i Q(v) x}\right) \wedge \varphi_{K M}(v, \tau, z) d \tau \\
& -\lim _{N \rightarrow \infty}\left(M_{T}(N, s) \varphi_{K M}(v, N, z)-M_{T}\left(N^{-1}, s\right) \varphi_{K M}\left(v, N^{-1}, z\right)\right) .
\end{aligned}
$$

Since $d d^{c}=-(2 \pi i)^{-1} \partial \bar{\partial}$, we see that to establish the claim it suffices to show that the second term in the right hand side vanishes. This follows for $z \notin \mathbb{D}_{v}$ from the asymptotic behavior of $M_{T}(y, s)$ given by (3-17) and (3-18). 
We can now express $\tilde{\Phi}(T, \varphi, s)_{K}$ as a regularized theta lift. Namely, for $T=$ $\left(\begin{array}{ll}a & b \\ b & c\end{array}\right) \in \operatorname{Sym}_{2}(F)$ totally positive definite, define a function

$$
\widetilde{\mathscr{M}}_{T}(n a, s): N(F) \backslash N(\mathbb{A}) \times A(\mathbb{R})^{0} \longrightarrow \mathbb{C}
$$

by

$$
\begin{aligned}
\widetilde{\mathscr{M}}_{T}\left(n m\left(y^{1 / 2}, t^{1 / 2}\right), s\right)=2 \kappa_{\operatorname{dim}(V)}^{-1} \overline{\psi_{T}(n)} & M_{\sigma_{1}(T)}\left(y_{1}, s\right) \tilde{M}_{\sigma_{1}(T)^{\iota}}\left(t_{1}, s\right) \\
& \times y_{1}^{1-\kappa / 2} t_{1}^{-\kappa / 2} \prod_{i=2}^{d} W_{a_{i}}\left(y_{i}\right) W_{c_{i}}\left(t_{i}\right) .
\end{aligned}
$$

We also need to specify a Schwartz form

$$
\tilde{\varphi}_{\infty} \in\left[\mathscr{S}\left(V(\mathbb{R})^{2}\right) \otimes \mathscr{A}^{1,1}(\mathbb{D})\right]^{H(\mathbb{R})}
$$

to define the regularized theta lift. Define

$$
\tilde{\varphi}^{1,1}(v, w, z)=\varphi^{0}(v, z) \cdot \varphi_{K M}(w, z) \in \mathscr{S}\left(V_{1}^{2}\right) \otimes \mathscr{A}^{1,1}(\mathbb{D})
$$

and

$$
\tilde{\varphi}_{\infty}(v, w, z)=\tilde{\varphi}^{1,1}\left(v_{1}, w_{1}, z\right) \otimes \varphi_{+}^{0}\left(v_{2}, w_{2}\right) \otimes \cdots \otimes \varphi_{+}^{0}\left(v_{d}, w_{d}\right),
$$

so that for every $g \in \operatorname{Sp}_{4}\left(\mathbb{A}_{F}\right)$ and $\varphi \in \mathscr{S}\left(V\left(\mathbb{A}_{f}\right)^{2}\right)$ fixed by $K$, the theta function

$$
\theta\left(g ; \varphi \otimes \tilde{\varphi}_{\infty}\right)_{K}=\sum_{(v, w) \in V(F)^{2}} \omega\left(g_{f}\right) \varphi(v, w) \cdot \omega\left(g_{\infty}\right) \tilde{\varphi}_{\infty}(v, w)
$$

defines a (1,1)-form on $X_{K}$. Given a measurable function $f: \mathrm{Sp}_{4}\left(\mathbb{A}_{F}\right) \rightarrow \mathbb{C}$ that satisfies $f(n g)=f(g)$ for all $n \in N(F)$, define

$$
\left(\widetilde{\mathscr{M}}_{T}(s), f\right)^{\mathrm{reg}}=\int_{A(\mathbb{R})^{0}} \int_{N(F) \backslash N(\mathbb{A})} \tilde{\mathscr{M}}_{T}(n a, s) f(n a) d n d a,
$$

provided that the integral converges. Then we have the identity

$$
\tilde{\Phi}(T, \varphi, s)_{K}=\left(\widetilde{\mathscr{M}}_{T}(s), \theta\left(\cdot ; \varphi \otimes \tilde{\varphi}_{\infty}\right)_{K}\right)^{\mathrm{reg}},
$$

valid in an open set $U \subset X_{K}$ whose complement has measure zero. This is proved in the same way as Proposition 3.21.

Here is the desired result that shows that one can evaluate $[\tilde{\Phi}(T, \varphi, s)]$ by interchanging the order of integration.

Proposition 3.27. Let $K \subset H\left(\mathbb{A}_{f}\right)$ be an open compact subgroup that fixes $\varphi$ and let $\alpha \in \mathscr{A}_{c}^{n-1, n-1}\left(X_{K}\right)$. Then, for $\operatorname{Re}(s) \gg 0$, we have $\left(\left[\tilde{\Phi}(T, \varphi, s)_{K}\right], \alpha\right)=\int_{A(\mathbb{R})^{0}} \int_{N(F) \backslash N(\mathbb{A})} \tilde{\mathscr{M}}_{T}(n a, s) \int_{X_{K}} \theta\left(n a ; \varphi \otimes \tilde{\varphi}_{\infty}\right)_{K} \wedge \alpha d n d a$. 
Proof. Performing the integration over $N(F) \backslash N(\mathbb{A})$, we find $\left(\left[\tilde{\Phi}(T, \varphi, s)_{K}\right], \alpha\right)=\int_{X_{K}} \int_{A(\mathbb{R})^{0}} \tilde{\mathscr{M}}_{T}(a, s) \sum_{(v, w) \in \Omega_{T}(F)} \varphi(v, w) \cdot \omega(a) \tilde{\varphi}_{\infty}(v, w, z) \wedge \alpha$, and we need to prove that this expression is absolutely convergent. Since $K$ has only finitely many orbits on the support of $\varphi$, it suffices to show that

$$
\int_{\Gamma_{v, w} \backslash \mathbb{D}^{+}} \int_{A(\mathbb{R})^{0}} \tilde{\mathscr{M}}_{T}(a, s) \cdot \omega(a) \tilde{\varphi}_{\infty}(v, w, z) \wedge \eta
$$

is absolutely convergent, for any vectors $v, w \in \Omega_{T}(F)$ and any compactly supported form $\eta \in \mathscr{A}_{c}^{n-1, n-1}\left(\Gamma \backslash \mathbb{D}^{+}\right)$. This will follow if we can show that

$$
\int_{\Gamma_{v, w} \backslash \mathbb{D}^{+}} \int_{A(\mathbb{R})^{0}}\left|\tilde{\mathscr{M}}_{T}(a, s)\right| \cdot\left\|\omega(a) \tilde{\varphi}_{\infty}(v, w, z)\right\| d a d \mu(z)<\infty,
$$

that is, we need to show that the inner integral in this expression yields an integrable function on $\Gamma_{v, w} \backslash \mathbb{D}^{+}$. Denote this inner integral by $f(v, w, z, s)$. Note that

$$
\left\|\tilde{\varphi}_{\infty}(v, w, z)\right\|=\sum_{i}\left\|P_{i}(w, z)\right\| \cdot \varphi^{0}(v, z) \varphi^{0}(w, z),
$$

where the sum over $i$ is finite and, for fixed $z$, the $P_{i}(w, z)$ are polynomials in $w$ (valued in differential forms). These polynomials satisfy $\left\|P_{i}(h w, h z)\right\|=\left\|P_{i}(w, z)\right\|$ for all $h \in H(\mathbb{R})$ and have degree 2; see [Kudla 1997, (7.16)]. Hence

$$
\left\|\tilde{\varphi}_{\infty}(v, w, z)\right\|<C \cdot Q\left(w_{z^{\perp}}\right) \cdot \varphi^{0}(v, z) \varphi^{0}(w, z),
$$

for some constant $C>0$. Using this estimate, we find that

$$
\begin{array}{ll}
f(v, w, z, s)=O\left(Q\left(v_{z^{\perp}}\right)^{-\frac{s+s_{0}}{2}} \cdot Q\left(w_{z^{\perp}}\right)^{-\frac{s+s_{0}}{2}}\right) & \text { if }\left|Q\left(v_{z}\right)\right|,\left|Q\left(w_{z}\right)\right|>\epsilon>0, \\
f(v, w, z, s)=O\left(\log \left(\left|Q\left(v_{z}\right)\right|\right) \cdot\left|Q\left(w_{z^{\perp}}\right)\right|^{-\frac{s+s_{0}}{2}+1}\right) & \text { as }\left|Q\left(v_{z}\right)\right| \rightarrow 0, \\
f(v, w, z, s)=O\left(\log \left(\left|Q\left(w_{z}\right)\right|\right) \cdot\left|Q\left(v_{z^{\perp}}\right)\right|^{-\frac{s+s_{0}}{2}}\right) & \text { as }\left|Q\left(w_{z}\right)\right| \rightarrow 0 .
\end{array}
$$

Since $f\left(v, w, h^{\prime} z, s\right)=f(v, w, z, s)$ for $h^{\prime} \in H^{\prime}(\mathbb{R})=\left(H_{v}\right)_{+}(\mathbb{R}) \cap\left(H_{w}\right)_{+}(\mathbb{R})$ and the quotient $\Gamma_{v, w} \backslash \mathbb{D}_{v, w}^{+}$has finite volume, the claim follows from these estimates by Lemma 3.8 applied to $H^{\prime}(\mathbb{R}) \backslash \mathbb{D}^{+}$.

Corollary 3.28. Let $K \subset H\left(\mathbb{A}_{f}\right)$ be an open compact subgroup that fixes $\varphi$ and let $\alpha \in \mathscr{A}_{c}^{n-1, n-1}\left(X_{K}\right)$ be a closed form. For $g \in \operatorname{Sp}_{4}\left(\mathbb{A}_{F}\right)$, write

$$
\theta(g ; \varphi, \alpha)=\int_{X_{K}} \theta\left(g ; \varphi \otimes \tilde{\varphi}_{\infty}\right) \wedge \alpha .
$$


Then

$$
\begin{aligned}
\left(\left[\Phi(T, \varphi)_{K}\right]-\right. & {\left.\left[\Phi\left(T^{\iota}, \varphi^{\iota}\right)_{K}\right], \alpha\right) } \\
= & \mathrm{CT}_{s=(n-1) / 2}\left[\left(\widetilde{\mathscr{M}}_{T}(s), \theta(\cdot ; \varphi, \alpha)\right)^{\mathrm{reg}}-\left(\tilde{\mathscr{M}}_{T^{\iota}}(s), \theta\left(\cdot ; \varphi^{\iota}, \alpha\right)\right)^{\mathrm{reg}}\right] .
\end{aligned}
$$

Proof. This follows from (3-83) and Proposition 3.27.

\section{An example: products of Shimura curves}

The goal of this section is to illustrate the main constructions and results above in one of the simplest cases: when the Shimura variety attached to $\operatorname{GSpin}(V)$ is a product of Shimura curves attached to a quaternion algebra $B$ over $\mathbb{Q}$. In this case, the currents in Section 3 can be described in the more familiar language of Hecke correspondences and $\mathrm{CM}$ points. We give this description in Section 4B.

Throughout this section, we fix an indefinite quaternion algebra $B$ over $\mathbb{Q}$; we assume that $B \nsubseteq M_{2}(\mathbb{Q})$. We write $S$ for the set of places where $B$ ramifies and $d(B)$ for the discriminant of $B$. Denote by $n: B \rightarrow F$ the reduced norm and let $V$ be $B$ endowed with the quadratic form given by $Q(v)=n(v)$. Then $(V, Q)$ is a nondegenerate quadratic space over $\mathbb{Q}$ with signature $(2,2)$ and $\chi_{V}=1$.

4A. Quaternion algebras and Shimura curves. The group $H=\mathrm{GSpin}(V)$ can in this case be described more concretely. Namely, consider $B^{\times}$as an algebraic group over $\mathbb{Q}$ defined by

$$
B^{\times}(R)=\left(B \otimes_{\mathbb{Q}} R\right)^{\times}
$$

for any $\mathbb{Q}$-algebra $R$ and let

$$
B^{\times} \times \mathrm{GL}_{1} B^{\times}=\left\{\left(g_{1}, g_{2}\right) \in B^{\times} \times B^{\times} \mid n\left(g_{1}\right)=n\left(g_{2}\right)\right\} .
$$

The group $B^{\times} \times B^{\times}$acts on $V$ by $\left(g_{1}, g_{2}\right) \cdot x=g_{1} x g_{2}^{-1}$. This induces an exact sequence

$$
1 \longrightarrow \mathbb{G}_{m} \longrightarrow B^{\times} \times_{\mathrm{GL}_{1}} B^{\times} \longrightarrow \mathrm{SO}(V) \longrightarrow 1
$$

showing that

$$
\mathrm{SO}(V) \cong \mathbb{G}_{m} \backslash\left(B^{\times} \times \mathrm{GL}_{1} B^{\times}\right), \quad \mathrm{GSO}(V) \cong \mathbb{G}_{m} \backslash\left(B^{\times} \times B^{\times}\right)
$$

and in fact one has

$$
H \cong B^{\times} \times \mathrm{GL}_{1} B^{\times} .
$$

The theory in Section 2 applies to this case. If we denote by $\mathbb{W}$ the Poincaré upper half plane, we have

$$
\mathbb{D}^{+} \cong \mathbb{H} \times \mathbb{H}
$$


Fix once and for all an isomorphism $\iota: B \otimes_{\mathbb{Q}} \mathbb{A}^{S} \cong M_{2}\left(\mathbb{A}^{S}\right)$. For $p \in S$, denote by $\mathscr{O}_{B, p}$ the maximal order of $B \otimes_{\mathbb{Q}} \mathbb{Q}_{p}$. Let

$$
\hat{\mathscr{O}}_{B}=\iota^{-1}\left(M_{2}\left(\prod_{p \notin S} \mathbb{Z}_{p}\right)\right) \times \prod_{p \in S} \mathscr{O}_{B, p}, \quad K_{B}=\hat{\mathscr{O}}_{B}^{\times} .
$$

Then $\hat{\mathscr{O}}_{B}$ is a maximal order of $B \otimes_{\mathbb{Q}} \mathbb{A}_{f}$ and $K_{B}$ is a maximal compact subgroup of $B\left(\mathbb{A}_{f}\right)^{\times}$.

Define the (full level) Shimura curve attached to $B$ to be

$$
X_{B, K}=B^{\times}(\mathbb{Q}) \backslash\left(\mathbb{M}^{ \pm} \times B^{\times}\left(\mathbb{A}_{f}\right)\right) / K .
$$

Then $X_{B, K}$ is the set of complex points of a complete curve $C_{K}$ defined over $\mathbb{Q}$. Let $K=\left(K_{B} \times K_{B}\right) \cap H\left(\mathbb{A}_{f}\right)$ and define the (full level) Shimura variety

$$
X_{K}=H(\mathbb{Q}) \backslash\left(\mathbb{D} \times H\left(\mathbb{A}_{f}\right)\right) / K .
$$

Thus $X_{B, K}$ is the set of complex points of the surface $C_{K} \times C_{K}$. By (2-8), the surface $X_{B, K}$ is connected.

Given $v \in V$ of positive norm and denoting by $W \subset V$ its orthogonal complement, we have

$$
H_{v}=\mathrm{GSpin}(W) \cong B^{\times}
$$

as algebraic groups over $\mathbb{Q}$. The special divisors $Z(v, h)_{K}$ are hence given by embedded Shimura curves in $X_{K}$.

4B. Examples of $(1,1)$-currents. Let us give some explicit examples of the currents introduced in Section 3 in the case when $X_{K}$ is a product of Shimura curves, in the more classical language of Hecke correspondences and CM points. Assume that $F=\mathbb{Q}$ for simplicity and denote by $d(B)=p_{1} \cdots p_{2 r}$ the discriminant of $B$. Let $\hat{\mathscr{O}}_{B}$ and $K_{B}=\hat{\mathscr{O}}_{B}^{\times}$be as in (4-7) and let $K=\left(K_{B} \times K_{B}\right) \cap H\left(\mathbb{A}_{f}\right)$. Then $\mathscr{O}_{B}=B \cap \hat{\mathscr{O}}_{B}$ is a maximal order in $B$. Denote by $\mathscr{O}_{B}^{1} \subset \mathscr{O}_{B}^{\times}$be the subgroup of units of reduced norm 1 . The group $\mathscr{O}_{B}^{1}$ acts on $\mathbb{H}$ through the embedding $\iota_{\infty}: \mathscr{O}_{B}^{1} \rightarrow \mathrm{SL}_{2}(\mathbb{R})$ and we conclude that

$$
X_{B, K} \cong \mathscr{O}_{B}^{1} \backslash \mathbb{M}=: X_{0}^{B}
$$

is the full level Shimura curve $X_{0}^{B}$ and that $X_{K}=X_{0}^{B} \times X_{0}^{B}$.

4B1. Special divisors. Consider the vector $v_{1}=1 \in B=V$ of norm 1 . Then the inclusion $H_{v_{1}} \subset H$ corresponds to the diagonal embedding $\Delta: B^{\times} \rightarrow B^{\times} \times \mathrm{GL}_{1} B^{\times}$ and hence the map $i_{v_{1}, 1, K}: X\left(v_{1}\right)_{K} \rightarrow X_{K}$ defined in (2-15) is just the diagonal

$$
\Delta: X_{0}^{B} \longrightarrow X_{0}^{B} \times X_{0}^{B} .
$$


More generally, suppose $v \in \mathscr{O}_{B}$ has reduced norm $d$ and consider the map $i_{v, 1, K}: X(v)_{K} \rightarrow X_{K}$. If $d$ equals a prime $p \nmid d(B)$ then the intersection $H_{v}(\mathbb{Q}) \cap K$ is an Eichler order $\mathscr{O}_{B}(p)$ of level $p$ in $B$ and the map $i_{v, 1, K}: X(v)_{K} \rightarrow X_{K}$ equals the map

$$
X_{0}^{B}(p) \longrightarrow X_{0}^{B} \times X_{0}^{B}
$$

whose image is the Hecke correspondence $T(p)$. Similarly, if $d$ is a divisor of $d(B)$, we obtain the graph of the Atkin-Lehner involution $w_{d}$.

4B2. Currents for connected cycles: $G(v, w)_{\Gamma}$ and $\left[\Phi(v, w)_{\Gamma}\right]$. Consider now $v, w \in B$ spanning a positive definite plane. To simplify matters, let us assume that $v=1$ and that $w \in \mathscr{O}_{B}$ is such that $R:=\mathbb{Z}[w]$ is the full ring of integers of an imaginary quadratic field $L=R \otimes_{\mathbb{Z}} \mathbb{Q}$; such an $R$ is then automatically optimally embedded in $\mathscr{O}_{B}$ (recall that an embedding $j: R \hookrightarrow \mathscr{O}_{B}$ is said to be optimal if $\left.j(L) \cap \mathscr{O}_{B}=j(R)\right)$. The diagram (1-3) in this case becomes

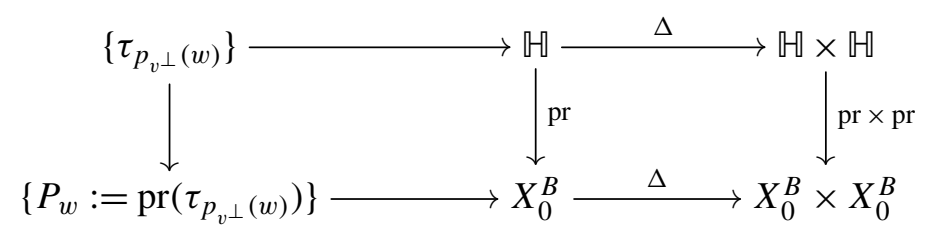

and $P_{w} \in X_{0}^{B}$ is a point with CM by $R$ (for one of the two CM-types of $R$ ). The function $G(v, w)_{\Gamma} \in \mathscr{C}^{\infty}\left(X_{0}^{B}-\left\{P_{w}\right\}\right)$ defined by (3-9) is a Green function for the divisor $\left[P_{w}\right] \in \operatorname{Div}\left(X_{0}^{B}\right)$; we denote this function by $G_{\left[P_{w}\right]}$ and the associated current in $\mathscr{D}^{0,0}\left(X_{0}^{B}\right)$ by $\left[G_{\left[P_{w}\right]}\right]$. The current $\left[\Phi(v, w)_{\Gamma}\right]$ in (3-33) is given by

$$
\left[\Phi(v, w)_{\Gamma]}\right]=2 \pi i \cdot \Delta_{*}\left(\left[G_{\left[P_{w}\right]}\right]\right),
$$

so that for $\alpha \in \mathscr{A}^{1,1}\left(X_{0}^{B} \times X_{0}^{B}\right)$ we have

$$
\left[\Phi(v, w)_{\Gamma}\right](\alpha)=2 \pi i \int_{X_{0}^{B}} G_{\left[P_{w}\right]} \cdot \Delta^{*}(\alpha) .
$$

4B3. The current $\left[\Phi(v, w, 1)_{K}\right]$. Our next goal is to write down an explicit example of the current $\left[\Phi(v, w, 1)_{K}\right]$ in (3-43). We have

$$
H_{v, w}=\operatorname{GSpin}(\mathbb{Q}\langle v, w\rangle)=L^{\times}
$$

as an algebraic group over $\mathbb{Q}$. The embeddings $H_{v, w} \rightarrow H_{v} \rightarrow H$ correspond to embeddings of algebraic groups

$$
L^{\times} \longrightarrow B^{\times} \stackrel{\Delta}{\longrightarrow} B^{\times} \times_{\mathrm{GL}_{1}} B^{\times},
$$

defined over $\mathbb{Q}$, where the second embedding is just the diagonal. Note that $H_{v, w}(\mathbb{R})=\left(K \otimes_{\mathbb{Q}} \mathbb{R}\right)^{\times}=\mathbb{C}^{\times}$with spinor norm the usual norm on $\mathbb{C}$. In particular, 
every element of this group has positive spinor norm and hence $\left(H_{v, w}\right)_{+}(\mathbb{R})=$ $H_{v, w}(\mathbb{R})$ and $\left(H_{v, w}\right)_{+}(\mathbb{Q})=H_{v, w}(\mathbb{Q})=L^{\times}$. Moreover, since $R \rightarrow \mathscr{O}$ is optimal, we have

$$
\left(H_{v, w}\right)_{+}(\mathbb{Q}) \backslash H_{v, w}\left(\mathbb{A}_{f}\right) / K_{U} \cong L^{\times} \backslash \mathbb{A}_{L, f}^{\times} / \hat{\mathscr{O}}_{L}^{\times}=\operatorname{Pic}\left(\mathscr{O}_{L}\right) .
$$

Let $\left\{h_{i}^{\prime} \mid i=1, \ldots, s\right\}$ be a set of representatives for this double coset and write $h_{i}^{\prime}=\gamma_{i} k_{i}$ with $\gamma_{i} \in H_{+}(\mathbb{Q})$ and $k_{i} \in K$. Note that we can find $\gamma_{i} \in\left(H_{v}\right)_{+}(\mathbb{Q})$ and $k_{i} \in K \cap H_{v}\left(\mathbb{A}_{f}\right)$. With such choices, we have

$$
\sum_{i}\left[\Phi\left(\gamma_{i}^{-1} v, \gamma_{i}^{-1} w\right)_{\Gamma}\right]=\sum_{i}\left[\Phi\left(v, \gamma_{i}^{-1} w\right)_{\Gamma}\right]
$$

The sum $\sum_{i}\left[\gamma_{i}^{-1} \cdot P_{w}\right]$ defines a divisor on $X_{0}^{B}$ of degree $h\left(\mathscr{O}_{L}\right)$. In fact, by Shimura's description of the Galois action, this divisor coincides with the orbit under $\operatorname{Gal}(H / L)$ of $P_{w} \in X_{0}^{B}(H)$, with $H$ the Hilbert class field of $L$. Hence we can write

$$
\sum_{i}\left[\gamma_{i}^{-1} P_{w}\right]=t_{H / L}\left[P_{w}\right]
$$

(Here $t_{H / L}$ stands for taking the trace from $H$ to $L$ ). Since in this case $n=2$, the current $\left[\Phi(v, w, 1)_{K}\right]$ involves an additional sum. Namely, we need to choose $\gamma_{0} \in H(\mathbb{Q})$ such that $\gamma_{0} \cdot \mathbb{D}_{U}^{+}=\mathbb{D}_{U}^{-}$; we can find such an element satisfying additionally that $\gamma_{0} \cdot v=v$ and $\gamma_{0} \cdot w=-w$. Now we have to find $k_{i_{0}} \in K$ and $\gamma_{i_{0}} \in H_{+}(\mathbb{Q})$ such that $\gamma_{0} h_{i}^{\prime}=\gamma_{i_{0}} k_{i_{0}}$. With our choice of $K$ this is easy to do explicitly: let $\epsilon \in \mathscr{O}_{B}^{\times}$be a unit of norm -1 ; such an element always exists by [Vignéras 1980, Corollary 5.9]. Then $(\epsilon, \epsilon) \in H(\mathbb{Q}) \cap K$. If $h_{i}^{\prime}=\gamma_{i} k_{i}$ as above, then we can choose $\gamma_{i_{0}}=\gamma_{0} \gamma_{i} \cdot(\epsilon, \epsilon)^{-1}$ and $k_{i_{0}}=(\epsilon, \epsilon) k_{i}$. Then we have $\gamma_{i_{0}}^{-1} \cdot v=v$ and $\gamma_{i_{0}}^{-1} \cdot w=-(\epsilon, \epsilon) \cdot \gamma_{i}^{-1} \cdot w$ and hence

$$
\sum_{i}\left[\Phi\left(\gamma_{i_{0}}^{-1} v, \gamma_{i_{0}}^{-1} w\right)_{\Gamma}\right]=\sum_{i}\left[\Phi\left(v,(\epsilon, \epsilon) \gamma_{i}^{-1} w\right)_{\Gamma}\right]
$$

since $\left[\Phi(v, w)_{\Gamma}\right]=\left[\Phi(v,-w)_{\Gamma}\right]$. By Shimura's reciprocity law [Ogg 1983, Equation (5)], if $P_{w^{\prime}}$ is the point of $X_{0}^{B}$ corresponding to $\mathbb{D}_{w^{\prime}} \subset \mathbb{D}=\mathbb{U}^{ \pm}$, then its complex conjugate $\bar{P}_{w^{\prime}}$ corresponds to $\mathbb{D}_{(\epsilon, \epsilon) \cdot w^{\prime}}$. It follows that

and hence

$$
\sum_{i}\left[\gamma_{i}^{-1} P_{w}\right]+\sum_{i}\left[\gamma_{i_{0}}^{-1} P_{w}\right]=t_{H / \mathbb{Q}}\left[P_{w}\right]
$$

$$
\left[\Phi(v, w, 1)_{K}\right]=2 \pi i \cdot \Delta_{*}\left(\left[G_{t_{H / \mathbb{Q}}\left[P_{w}\right]}\right]\right),
$$

with $G_{t_{H / \mathbb{Q}}\left[P_{w}\right]}$ a Green function for the divisor $t_{H / \mathbb{Q}}\left[P_{w}\right]$ on $X_{0}^{B}$. 
4B4. The current $\left[\Phi(T, \varphi)_{K}\right]$. Consider now an order $R=\mathbb{Z}[\alpha]$ in an imaginary quadratic field $L \subset \mathbb{C}$ and let $x^{2}-t x+n$ be the minimal polynomial of $\alpha$. We assume that $L$ admits an embedding into $B$ and (for simplicity) that $(d(L), d(B))=1$ and that $R=\mathscr{O}_{L}$ is the ring of integers of $L$. Define

$$
\begin{aligned}
T & =\left(\begin{array}{cc}
1 & t / 2 \\
t / 2 & n
\end{array}\right), \\
\varphi_{\mathscr{O}_{B}^{2}} & =\text { characteristic function of } \hat{\mathscr{O}}_{B}^{2},
\end{aligned}
$$

and let us describe the current $\left[\Phi\left(T, \varphi_{\mathscr{O}_{B}^{2}}\right)\right]$ in (3-49). To do so, we need to describe the set of $K$-cosets of $\operatorname{Supp}\left(\varphi_{\mathscr{O}_{B}^{2}}\right) \cap \Omega_{T}\left(\mathbb{A}_{f}\right)$. We have

$$
\begin{aligned}
K \backslash\left[\operatorname{Supp}\left(\varphi_{\mathscr{O}_{B}^{2}}\right) \cap \Omega_{T}\left(\mathbb{A}_{f}\right)\right] & =\left(\hat{\mathscr{O}}_{B}^{\times} \times \hat{\mathbb{Z}}_{\times} \hat{\mathscr{O}}_{B}^{\times}\right) \backslash \Omega_{T}\left(\hat{\mathscr{O}}_{B}^{2}\right) \\
& =\prod_{v \nmid \infty}\left(\mathscr{O}_{B, v}^{\times} \times_{\mathbb{Z}_{v}^{\times}} \mathscr{O}_{B, v}^{\times}\right) \backslash \Omega_{T}\left(\mathscr{O}_{B, v}^{2}\right) .
\end{aligned}
$$

Note that the assignment $j \mapsto j(\alpha)$ induces a bijection between the (optimal) embeddings $j: R \rightarrow \mathscr{O}_{B}$ and the set of elements $w \in \mathscr{O}_{B}$ with $t(w)=t$ and $n(w)=n$, and this statement holds true locally too. It follows that the map $(1, w) \mapsto w$ induces a one-to-one correspondence

$$
\left(\mathscr{O}_{B, v}^{\times} \times \mathbb{Z}_{v}^{\times} \mathscr{O}_{B, v}^{\times}\right) \backslash \Omega_{T}\left(\mathscr{O}_{B, v}^{2}\right) \longleftrightarrow\left\{j: R \rightarrow \mathscr{O}_{B, v} \text { optimal }\right\} / \mathscr{O}_{B, v}^{\times},
$$

where the equivalence in the right-hand side is with respect to conjugation by $\mathscr{O}_{B, v}^{\times}$. The set on the right-hand side has cardinality 1 if $B_{v} \cong M_{2}\left(\mathbb{Q}_{v}\right)$ and 2 if $B_{v}$ is division; moreover, in the latter case the local Atkin-Lehner involution permutes the two elements (see [Vignéras 1980, Theorems II.3.1, II.3.2]). Hence the set

$$
K \backslash\left[\operatorname{Supp}\left(\varphi_{\mathscr{O}_{B}^{2}}\right) \cap \Omega_{T}\left(\mathbb{A}_{f}\right)\right]
$$

is a torsor under the Atkin-Lehner group $W_{B}$. Since the set $\operatorname{CM}\left(\mathscr{O}_{L}\right)$ of points in $X_{0}^{B}$ with $\mathrm{CM}$ by $\mathscr{O}_{L}$ is a torsor under $\operatorname{Pic}\left(\mathscr{O}_{L}\right) \times W_{B}$, we conclude that

$$
\left[\Phi\left(\left(\begin{array}{cc}
1 & t / 2 \\
t / 2 & n
\end{array}\right), \varphi_{\mathscr{O}_{B}^{2}}\right)_{K}\right]=2 \pi i \cdot\left(X_{0}^{B} \stackrel{\Delta}{\rightarrow} X_{0}^{B} \times X_{0}^{B}\right)_{*}\left(\left[G_{t_{L / \mathbb{Q}}[}\left[\mathrm{CM}\left(\mathscr{O}_{L}\right)\right]\right]\right)
$$

is the pushforward along the diagonal of a Green current $\left[G_{t_{L / \mathbb{Q}}\left[\mathrm{CM}\left(\mathscr{O}_{L}\right)\right]}\right]$ for the divisor $t_{L / \mathbb{Q}}\left[\mathrm{CM}\left(\mathscr{O}_{L}\right)\right]$.

Note that by choosing $\varphi \in \mathscr{S}\left(V\left(\mathbb{A}_{f}\right)^{2}\right)$ to have support in a single $K$-orbit of (4-27), we recover all the currents of the form (4-23).

4B5. The current $\left[\Phi(T, \varphi)_{K}\right]-\left[\Phi\left(T^{\iota}, \varphi^{\iota}\right)_{K}\right]$. Recall that we have defined an involution $\iota$ on the set of pairs $(T, \varphi)$, given by (3-82). Our next goal is to give an example of the action of $\iota$. 
Let $p$ be a prime, $p \equiv 1(\bmod 4)$ and not dividing $d(B)$, and define

$$
\begin{aligned}
T & =\left(\begin{array}{ll}
1 & \\
& p
\end{array}\right), \\
\varphi_{\mathscr{O}_{B}^{2}} & =\text { characteristic function of } \hat{\mathscr{O}}_{B}^{2} .
\end{aligned}
$$

The previous computation of $\left[\Phi\left(T, \varphi_{\mathscr{O}_{B}^{2}}\right)\right]$ shows that this current is supported on the diagonal $\Delta$, and more precisely that

$$
\left[\Phi\left(\left(\begin{array}{cc}
1 & \\
& p
\end{array}\right), \varphi_{\mathscr{O}_{B}^{2}}\right)_{K}\right]=2 \pi i \cdot\left(X_{0}^{B} \stackrel{\Delta}{\rightarrow} X_{0}^{B} \times X_{0}^{B}\right)_{*}\left(\left[G_{t_{L / \mathbb{Q}}}[\mathrm{CM}(\mathbb{Z}[\sqrt{-p}])]\right) .\right.
$$

Note that $\varphi_{\mathscr{O}_{B}^{2}}^{l}=\varphi_{\mathscr{O}_{B}^{2}}$ and that $T^{\iota}=\left(\begin{array}{c}p_{1} \\ { }_{1}\end{array}\right)$. In particular, the current $\left[\Phi\left(T^{\iota}, \varphi_{\mathscr{O}_{B}^{2}}^{\iota}\right)\right]$ is different from $\left[\Phi\left(T, \varphi_{\mathscr{O}_{B}^{2}}\right)\right]$, as the former is supported on the Hecke correspondence $T(p)$. More precisely, the same argument as above, with trivial modifications, shows that

$$
\left[\Phi\left(\left(\begin{array}{ll}
p & \\
& 1
\end{array}\right), \varphi_{\mathscr{O}_{B}^{2}}\right)_{K}\right]=2 \pi i \cdot\left(X_{0}^{B}(p) \rightarrow X_{0}^{B} \times X_{0}^{B}\right)_{*}\left(\left[G_{t_{L / \mathbb{Q}}}[\mathrm{CM}(\mathbb{Z}[\sqrt{-p}])]\right),\right.
$$

where here $[\mathrm{CM}(\mathbb{Z}[\sqrt{-p}])]$ denotes the divisor consisting of all points in $X_{0}^{B}(p)$ with $\mathrm{CM}$ by $\mathbb{Z}[\sqrt{-p}]$ (for some $\mathrm{CM}$ type of $\mathbb{Z}[\sqrt{-p}]$ ).

\section{Acknowledgments}

Most of the work on this paper was done during my Ph.D. at Columbia University, as part of my Ph.D. thesis. I would like to express my deep gratitude to my advisor Shou-Wu Zhang, for introducing me to this area of mathematics, for his guidance and encouragement and for many very helpful suggestions. I would also like to thank Stephen S. Kudla for answering my questions about the theta correspondence and for several very inspiring remarks and conversations. This paper has also benefited from comments and discussions with Patrick Gallagher, Yifeng Liu, André Neves, Ambrus Pál, Yiannis Sakellaridis and Wei Zhang; I am grateful to all of them. Finally, I want to thank the referee for his useful suggestions and comments.

\section{References}

[Beilinson 1984] A. A. Beilinson, "Vysshie regulatory i znacheniya L-funktsiì", Itogi Nauki i Tekhniki Ser. Sovrem. Probl. Mat. Nov. Dost. 24 (1984), 181-238. Translated as "Higher regulators and values of $L$-functions", Soviet Math. 20:2 (1985), 2036-2070. MR 86h:11103 Zbl 0588.14013

[Borcherds 1998] R. E. Borcherds, "Automorphic forms with singularities on Grassmannians", Invent. Math. 132:3 (1998), 491-562. MR 99c:11049 Zbl 0919.11036

[Borcherds 1999] R. E. Borcherds, "The Gross-Kohnen-Zagier theorem in higher dimensions", Duke Math. J. 97:2 (1999), 219-233. MR 2000f:11052 Zbl 0967.11022

[Borel 1969] A. Borel, Introduction aux groupes arithmétiques, Actualités Scientifiques et Industrielles 1341, Hermann, Paris, 1969. MR 39 \#5577 Zbl 0186.33202 
[Bridson and Haefliger 1999] M. R. Bridson and A. Haefliger, Metric spaces of non-positive curvature, Grundlehren der Mathematischen Wissenschaften 319, Springer, Berlin, 1999. MR 2000k:53038 Zbl 0988.53001

[Bruinier 2002] J. H. Bruinier, Borcherds products on $O(2, l)$ and Chern classes of Heegner divisors, Lecture Notes in Mathematics 1780, Springer, Berlin, 2002. MR 2003h:11052 Zbl 1004.11021

[Bruinier 2012] J. H. Bruinier, "Regularized theta lifts for orthogonal groups over totally real fields", J. Reine Angew. Math. 672 (2012), 177-222. MR 2995436 Zbl 1268.11058

[Bruinier and Funke 2004] J. H. Bruinier and J. Funke, "On two geometric theta lifts", Duke Math. J. 125:1 (2004), 45-90. MR 2005m:11089 Zbl 1088.11030

[Chavel 2006] I. Chavel, Riemannian geometry: a modern introduction, 2-nd ed., Cambridge Studies in Advanced Mathematics 98, Cambridge University Press, 2006. MR 2006m:53002 Zbl 1099.53001

[Erdélyi et al. 1954] A. Erdélyi, W. Magnus, F. Oberhettinger, and F. G. Tricomi, Tables of integral transforms, vol. I, McGraw-Hill, New York, 1954. MR 15,868a Zbl 0055.36401

[Goncharov 2005] A. B. Goncharov, "Regulators", pp. 295-349 in Handbook of $K$-theory, vol. 1, edited by E. M. Friedlander and D. R. Grayson, Springer, Berlin, 2005. MR 2006j:11092 Zbl 1101. 19004

[Kudla 1997] S. S. Kudla, "Algebraic cycles on Shimura varieties of orthogonal type", Duke Math. J. 86:1 (1997), 39-78. MR 98e:11058 Zbl 0879.11026

[Kudla and Millson 1986] S. S. Kudla and J. J. Millson, "The theta correspondence and harmonic forms, I”, Math. Ann. 274:3 (1986), 353-378. MR 88b:11023 Zbl 0594.10020

[Kudla and Millson 1987] S. S. Kudla and J. J. Millson, "The theta correspondence and harmonic forms, II”, Math. Ann. 277:2 (1987), 267-314. MR 89b:11041 Zbl 0618.10022

[Kudla and Millson 1988] S. S. Kudla and J. J. Millson, "Tubes, cohomology with growth conditions and an application to the theta correspondence", Canad. J. Math. 40:1 (1988), 1-37. MR 90k:11054 Zbl 0652.10021

[Kudla and Millson 1990] S. S. Kudla and J. J. Millson, "Intersection numbers of cycles on locally symmetric spaces and Fourier coefficients of holomorphic modular forms in several complex variables”, Inst. Hautes Études Sci. Publ. Math. 71 (1990), 121-172. MR 92e:11035 Zbl 0722.11026

[Kudla and Rallis 1988] S. S. Kudla and S. Rallis, "On the Weil-Siegel formula", J. Reine Angew. Math. 387 (1988), 1-68. MR 90e:11059 Zbl 0644.10021

[Lebedev 1965] N. N. Lebedev, Special functions and their applications, edited by R. A. Silverman, Prentice-Hall, Englewood Cliffs, NJ, 1965. MR 30 \#4988 Zbl 0131.07002

[Oda and Tsuzuki 2003] T. Oda and M. Tsuzuki, "Automorphic Green functions associated with the secondary spherical functions”, Publ. Res. Inst. Math. Sci. 39:3 (2003), 451-533. MR 2004f:11046 Zbl 1044.11033

[Ogg 1983] A. P. Ogg, "Real points on Shimura curves", pp. 277-307 in Arithmetic and geometry, vol. I, edited by M. Artin and J. Tate, Progr. Math. 35, Birkhäuser, Boston, 1983. MR 85m:11034 Zbl 0531.14014

[Vignéras 1980] M.-F. Vignéras, Arithmétique des algèbres de quaternions, Lecture Notes in Mathematics 800, Springer, Berlin, 1980. MR 82i:12016 Zbl 0422.12008

[Vogan and Zuckerman 1984] D. A. Vogan, Jr. and G. J. Zuckerman, "Unitary representations with nonzero cohomology", Compositio Math. 53:1 (1984), 51-90. MR 86k:22040 Zbl 0692.22008

[Voisin 2002] C. Voisin, "Nori's connectivity theorem and higher Chow groups", J. Inst. Math. Jussieu 1:2 (2002), 307-329. MR 2003m:14014 Zbl 1036.14004 
Communicated by Peter Sarnak

Received 2015-05-08 Revised 2015-12-09 Accepted 2016-02-04

Igarcia@math.toronto.edu

Current address:
Department of Mathematics, South Kensington Campus, Imperial College London, London, SW7 2AZ, United Kingdom Department of Mathematics, University of Toronto, 40 St. George Street, BA 6290, Toronto, ON M5S 2E4, Canada 


\section{Algebra \& Number Theory}

msp.org/ant

\section{EDITORS}

MANAGING EDITOR

Bjorn Poonen

Massachusetts Institute of Technology

Cambridge, USA

\author{
EDITORIAL BOARD CHAIR \\ David Eisenbud \\ University of California \\ Berkeley, USA
}

\section{BOARD OF EDITORS}

Georgia Benkart

Dave Benson

Richard E. Borcherds

John H. Coates

J-L. Colliot-Thélène

Brian D. Conrad

Hélène Esnault

Hubert Flenner

Sergey Fomin

Edward Frenkel

Andrew Granville

Joseph Gubeladze

Roger Heath-Brown

Craig Huneke

Kiran S. Kedlaya

János Kollár

Yuri Manin

Philippe Michel
University of Wisconsin, Madison, USA

University of Aberdeen, Scotland

University of California, Berkeley, USA

University of Cambridge, UK

CNRS, Université Paris-Sud, France

Stanford University, USA

Freie Universität Berlin, Germany

Ruhr-Universität, Germany

University of Michigan, USA

University of California, Berkeley, USA

Université de Montréal, Canada

San Francisco State University, USA

Oxford University, UK

University of Virginia, USA

Univ. of California, San Diego, USA

Princeton University, USA

Northwestern University, USA

École Polytechnique Fédérale de Lausanne
Susan Montgomery

Shigefumi Mori

Raman Parimala

Jonathan Pila

Anand Pillay

Victor Reiner

Peter Sarnak

Joseph H. Silverman

Michael Singer

Vasudevan Srinivas

J. Toby Stafford

Ravi Vakil

Michel van den Bergh

Marie-France Vignéras

Kei-Ichi Watanabe

Efim Zelmanov

Shou-Wu Zhang
University of Southern California, USA

RIMS, Kyoto University, Japan

Emory University, USA

University of Oxford, UK

University of Notre Dame, USA

University of Minnesota, USA

Princeton University, USA

Brown University, USA

North Carolina State University, USA

Tata Inst. of Fund. Research, India

University of Michigan, USA

Stanford University, USA

Hasselt University, Belgium

Université Paris VII, France

Nihon University, Japan

University of California, San Diego, USA

Princeton University, USA

\section{PRODUCTION}

production@msp.org

Silvio Levy, Scientific Editor

See inside back cover or msp.org/ant for submission instructions.

The subscription price for 2016 is US \$/year for the electronic version, and \$/year (+\$, if shipping outside the US) for print and electronic. Subscriptions, requests for back issues and changes of subscribers address should be sent to MSP.

Algebra \& Number Theory (ISSN 1944-7833 electronic, 1937-0652 printed) at Mathematical Sciences Publishers, 798 Evans Hall \#3840, c/o University of California, Berkeley, CA 94720-3840 is published continuously online. Periodical rate postage paid at Berkeley, CA 94704, and additional mailing offices.

ANT peer review and production are managed by EditFLOW ${ }^{\circledR}$ from MSP.

\section{PUBLISHED BY}

mathematical sciences publishers

nonprofit scientific publishing

http://msp.org/

(C) 2016 Mathematical Sciences Publishers 


\section{Algebra \& Number Theory}

Volume $10 \quad$ No. $3 \quad 2016$

Group schemes and local densities of ramified hermitian lattices in residue characteristic 2: Part I

SUNGMUN CHO

Presentation of affine Kac-Moody groups over rings

DANIEL ALLCOCK

Discriminant formulas and applications

Kenneth Chan, Alexander A. Young and James J. Zhang

LUIS E. GARCIA

Multiple period integrals and cohomology

RoElof W. BRUGGEMAN and YoungJu CHOIE

The existential theory of equicharacteristic henselian valued fields

SYLVY ANSCOMBE and ARNO FEHM

SOMNATH JHA, TADASHI OCHIAI and GERGELY ZÁBRÁDI 